Aus der Klinik für Allgemein-, Viszeral- und Kinderchirurgie

(Prof. Dr. med. B. M. Ghadimi)

der Medizinischen Fakultät der Universität Göttingen

\title{
Auswirkungen eines geführten perioperativen Behandlungskonzeptes bei ambulant zu operierenden Kindern auf die prä- und postoperative Befindlichkeit
}

\author{
INAUGURAL-DISSERTATION \\ zur Erlangung des Doktorgrades \\ der Medizinischen Fakultät der
}

Georg-August-Universität zu Göttingen

vorgelegt von

Annkathrin Feischen, geborene Peters

aus

Münster

Göttingen 2021 
Dekan:

\section{Betreuungsausschuss}

Betreuer/in

Ko-Betreuer/in:

\section{Prüfungskommission}

Referent/in

Ko-Referent/in:
Prof. Dr. med. W. Brück

Prof. Dr. B. M. Ghadimi

Prof. Dr. M. Bauer

Datum der mündlichen Prüfung: 03.03.2021 
Hiermit erkläre ich, die Dissertation mit dem Titel "Auswirkungen eines geführten perioperativen Behandlungskonzeptes bei ambulant zu operierenden Kindern auf die prä- und postoperative Befindlichkeit" eigenständig angefertigt und keine anderen als die von mir angegebenen Quellen und Hilfsmittel verwendet zu haben.

Göttingen, den 20.02.2021 


\section{Inhaltsverzeichnis}

Abbildungsverzeichnis .......................................................................................... III

Tabellenverzeichnis................................................................................................

Abkürzungsverzeichnis ............................................................................................... V

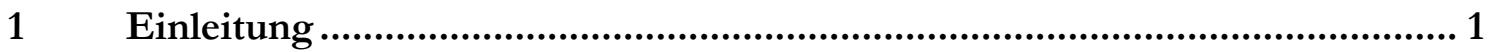

1.1 Perioperatives Management im ambulanten Setting ................................................................ 1

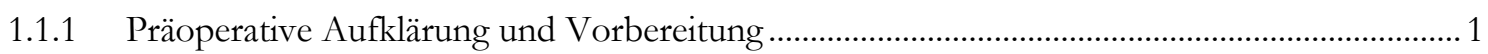

1.1.2 Anästhesie im ambulanten Setting ………………......................................................... 3

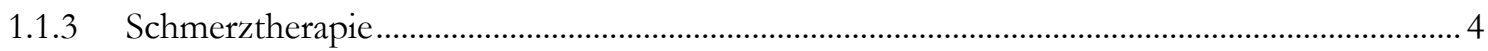

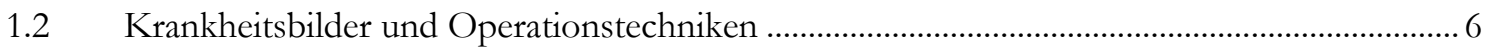

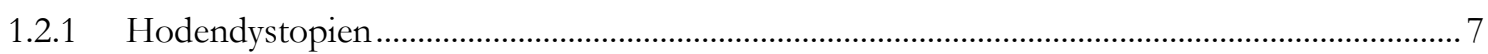

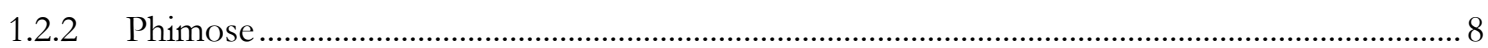

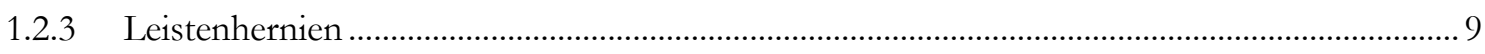

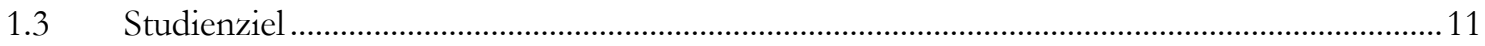

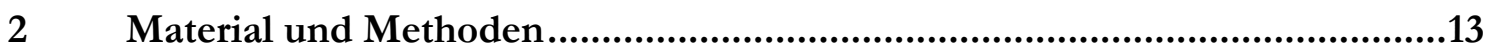

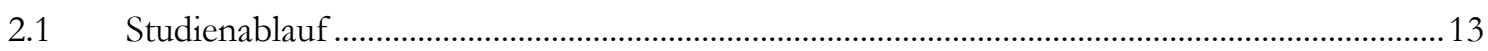

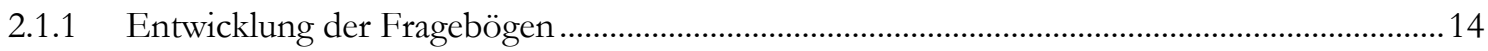

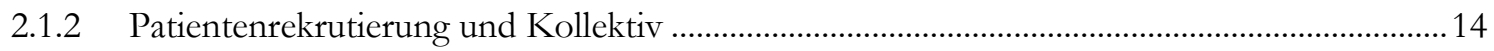

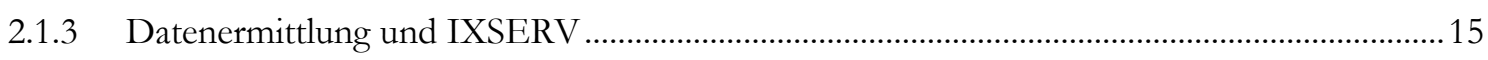

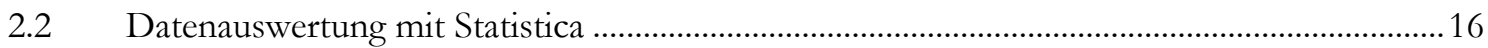

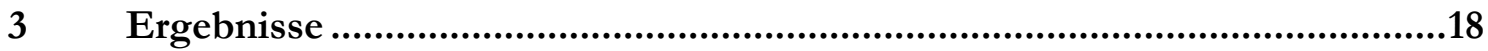

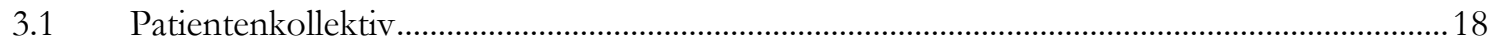

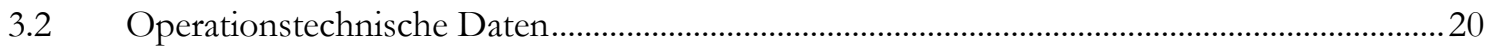

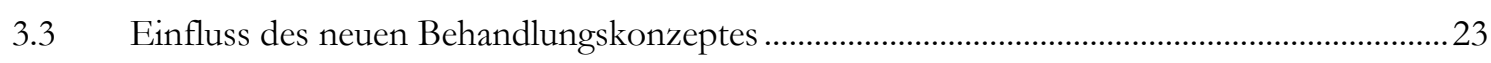

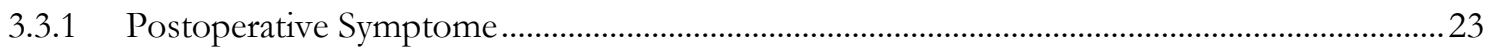

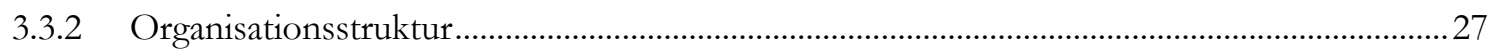

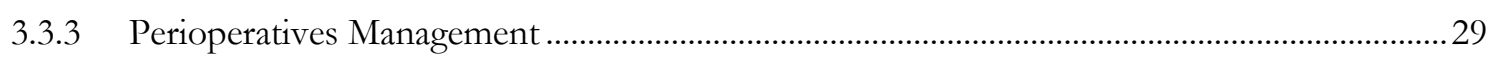

3.4 Korrelationsanalyse und Einfluss des neuen Behandlungskonzeptes ........................................35

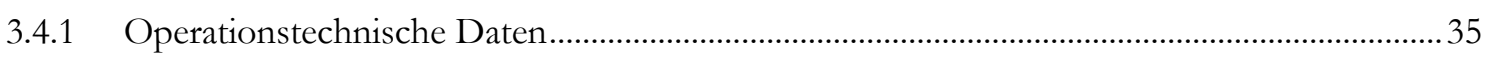

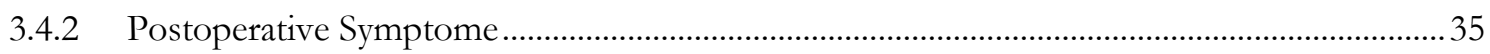

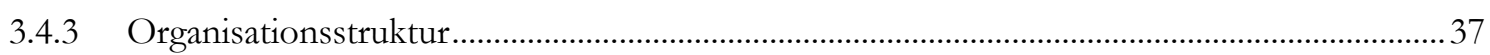

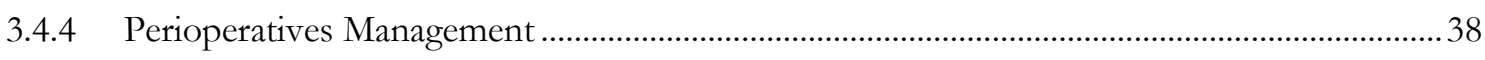

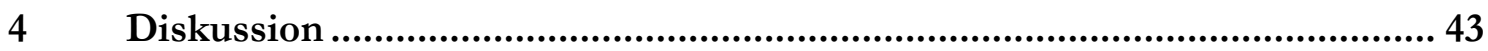

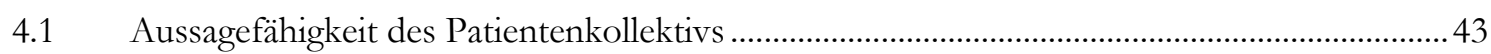

4.2 Interpretation und Fehleranalyse der Ergebnisse ......................................................................... 44 


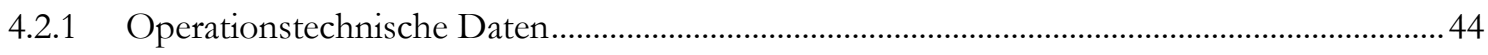

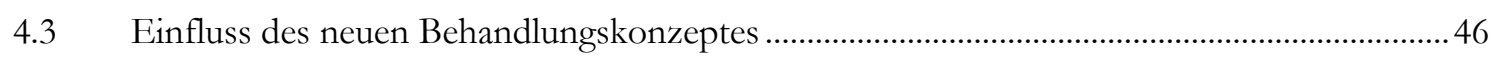

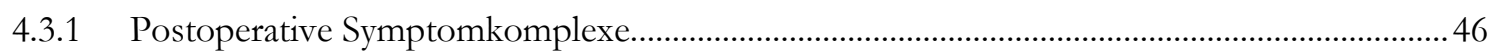

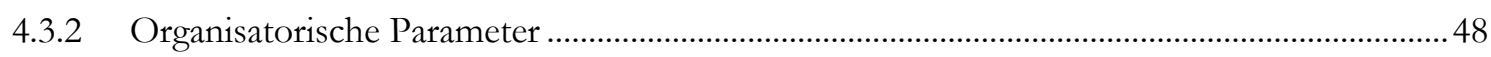

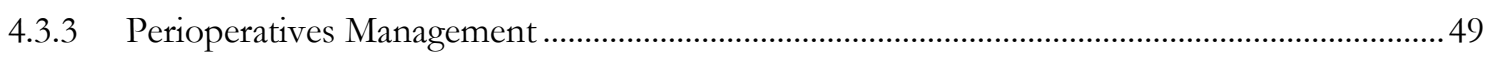

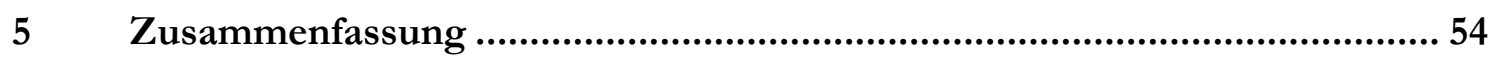

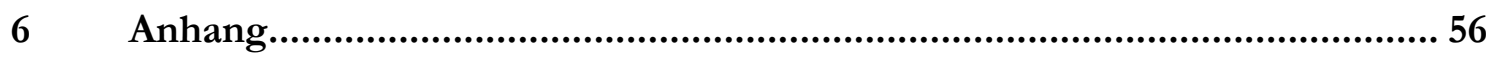

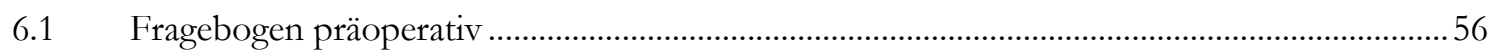

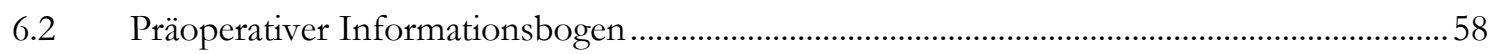

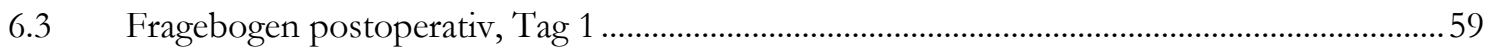

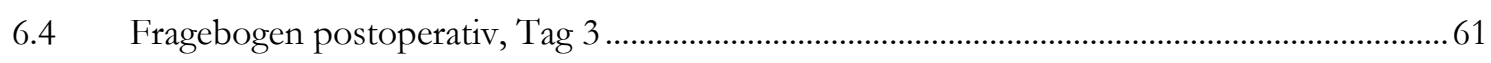

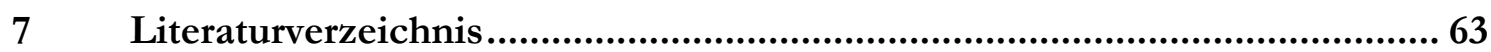

Lebenslauf

Fehler! Textmarke nicht definiert. 


\section{Abbildungsverzeichnis}

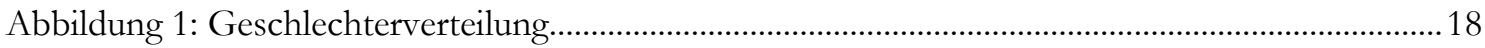

Abbildung 2: Altersverteilung abhängig vom Geschlecht. ............................................................... 19

Abbildung 3: Gewichtsverteilung aller untersuchten Kinder............................................................20

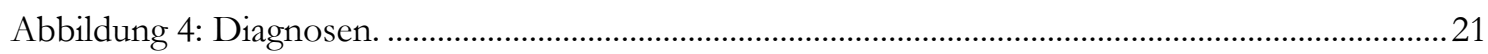

Abbildung 5: Anteil der verwendeten Peniswurzelblöcke an der Regionalanästhesie ( $0=$ nein, $1=\mathrm{ja})$.

Abbildung 6: Anteil der verwendeten Kaudalanästhesien an der Regionalanästhesien $(0=$ nein, $1=\mathrm{ja})$.

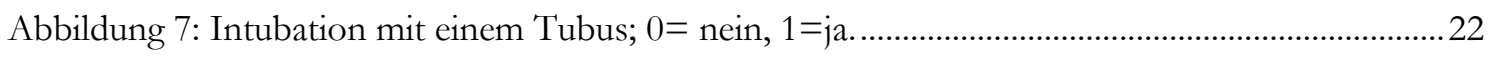

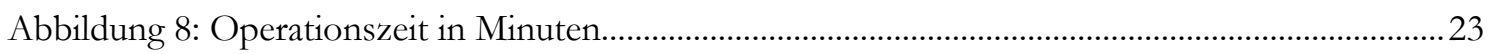

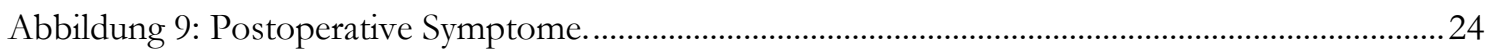

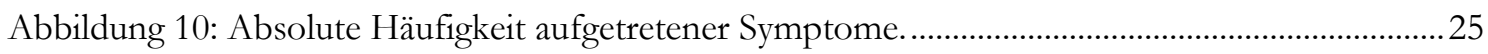

Abbildung 11: Schmerzangaben anhand der Schmerzskala $(0=$ kein Schmerz bis $5=$ unerträglicher Schmerz).

Abbildung 12: Zufriedenheit mit der Struktur des Operationstages $(0=$ keine Angabe, $1=$ trifft gar nicht zu, $2=$ trifft eher nicht zu, $3=$ unentschieden, $4=$ trifft eher zu, $5=$ trifft völlig zu).

Abbildung 13: Zusammenfassende Bewertung der präoperativen Aufklärung $(0=$ keine Angabe, $1=$ trifft gar nicht zu, $2=$ trifft eher nicht zu, $3=$ unentschieden, $4=$ trifft eher zu, 5= trifft völlig zu).....

Abbildung 14: Zufriedenheit mit der Aufklärung bezüglich möglicher Schmerzen $(0=$ keine Angabe, $1=$ trifft gar nicht $\mathrm{zu}, 2=$ trifft eher nicht zu, $3=$ unentschieden, $4=$ trifft eher zu, 5= trifft völlig zu).

Abbildung 15: Zufriedenheit mit der Aufklärung bezüglich möglicher Nachblutungen $(0=$ keine Angabe, $1=$ trifft gar nicht zu, $2=$ trifft eher nicht zu, $3=$ unentschieden, $4=$ trifft eher zu, $5=$ trifft völlig zu).

Abbildung 16: Zufriedenheit mit der Aufklärung über das Narkoseverfahren $(0=$ nein, $1=$ ja).......33

Abbildung 17: Zustimmung mit einer Nachsorgeuntersuchung durch den Kinderarzt $(0=$ nicht gewünscht, $1=$ gewünscht).

Abbildung 18: Subjektive Schmerzintensität in Bezug auf die Zeit bis zur Beschwerdefreiheit in Stunden dargestellt als Kaplan-Meier-Diagramm.

Abbildung 19: Zufriedenheit mit dem Ablauf des Operationstages vor Einführung des neuen Behandlungsplans $(0=$ nein, $1=j a)$.

Abbildung 20: Zufriedenheit mit dem Ablauf des Operationstages nach Einführung des neuen Behandlungsplans $(0=$ nein, $1=j a)$.

Abbildung 21: Zufriedenheit mit dem Ausmaß der Aufklärung vor Einführung des neuen Behandlungsplans $(1=$ trifft gar nicht zu, $2=$ trifft eher nicht zu, $3=$ unentschieden, $4=$ trifft eher zu, $5=$ trifft völlig zu).

Abbildung 22: Zufriedenheit mit dem Ausmaß der Aufklärung nach Einführung des neuen Behandlungsplans $(1=$ trifft gar nicht zu, $2=$ trifft eher nicht $\mathrm{zu}, 3=$ unentschieden, $4=$ trifft eher zu, $5=$ trifft völlig zu).

Abbildung 23: Zufriedenheit mit der Versorgung des Kindes im Krankenhaus vor Einführung des neuen Behandlungsplans $(1=$ trifft gar nicht zu, $2=$ trifft eher nicht zu, $3=$ unentschieden, $4=$ trifft eher zu, $5=$ trifft völlig zu).

Abbildung 24: Zufriedenheit mit der Versorgung des Kindes im Krankenhaus nach Einführung des Behandlungsplans ( $1=$ trifft gar nicht $\mathrm{zu}, 2=$ trifft eher nicht $\mathrm{zu}, 3=$ unentschieden, $4=$ trifft eher zu, $5=$ trifft völlig zu). 


\section{Tabellenverzeichnis}

Tabelle 1: Zuordnung Antwort - codierte Nummer. 15

Tabelle 2: Quellen der Datenerhebung...... 


\section{Abkürzungsverzeichnis}

NSAIDs

PONV

SSW

TIVA nonsteroidal anti-inflammatory drugs

postoperative nausea and vomiting

Schwangerschaftswoche

totale intravenöse Anästhesie 


\section{$1 \quad$ Einleitung}

\subsection{Perioperatives Management im ambulanten Setting}

Das ambulante Operieren im Kindesalter hat eine sehr große Bedeutung. Innerhalb der letzten zehn Jahre ist die Zahl der ambulanten kinderchirurgischen Interventionen von 26 auf 38 je tausend Kinder angestiegen. Urologische Eingriffe, wie die Orchidopexie, Zirkumzision, Hydrozelenresektion und die Herniotomie gehören mit zu den häufigsten ambulanten Operationen (Stein et al. 2017). Krankenhausaufenthalte und speziell Operationen können für Kinder und ihre Eltern belastend sein und bieten ein hohes Stresspotential. Angst und Stress können sowohl das psychische als auch das physische Wohlbefinden des Kindes negativ beeinflussen und dessen postoperative Genesung verzögern (Li et al. 2007). Schon zur Geburtsstunde der ambulanten Kinderchirurgie gegen Ende des 19. Jahrhunderts äußerte der Chirurg J. H. Nicoll aus Glasgow die Vermutung, dass die postoperative Versorgung der Kinder durch die Mutter der stationären Versorgung überlegen sei. In den späten 40er Jahren gerieten die psychischen Folgen von chirurgischen Eingriffen, Anästhesie und stationären Krankenhausaufenthalten zunehmend in den Fokus der Gesellschaft (Steward 2014). Aber nicht nur die Kinder werden durch eine Operation aus ihrer Routine gerissen. Durch Operationen im ambulanten Setting wird den Eltern eine völlig neue Verantwortung für die Gesundheit ihres Kindes übertragen (Li et al. 2007). Ein komplexes perioperatives Management ist vor diesem Hintergrund sehr wichtig, um die Eltern vor der Operation ausreichend zu informieren und zu beruhigen, damit dies indirekt auch die Kinder positiv beeinflusst (Karl et al. 2016)

Ein weiterer Vorteil der ambulanten operativen Versorgung gegenüber der vollstationären Versorgung beruht auf ökonomischen Faktoren. Fabricant et al. verglichen 2016 die anfallenden Kosten stationärer und ambulanter operativer Versorgungen in der Kinderorthopädie. In allen acht untersuchten Operationsverfahren konnte ein nachweislicher Kostenvorteil von 17 bis 43 Prozent zugunsten der ambulanten Versorgung nachgewiesen werden.

\subsubsection{Präoperative Aufklärung und Vorbereitung}

Die Unzufriedenheit der Eltern, perioperative Komplikationen und Unstimmigkeiten werden selten durch die eigentliche Operation verursacht. Deutlich häufiger liegt die Problematik in der Organisation des ambulanten Operationstages oder innerhalb der Korrespondenz zwischen Eltern und dem medizinischen Personal. Um dies zu vermeiden, ist eine Interaktion aller beteiligten Instanzen entscheidend (Stark und Steffens 2006). 
Nachweislich existieren ebenfalls verschiedene Einflussfaktoren, die die Reaktion des Kindes auf den Krankenhausaufenthalt, dauert dieser auch nur ein Tag, beeinflussen. Beschrieben sind hier die eigene altersabhängige Anpassungsfähigkeit des Kindes sowie die Unterstützung, die es erfährt (Lynch 1994). Letzteres ist ein Punkt, der zum Teil durch eine suffiziente präoperative Vorbereitung beeinflusst werden kann. Die ungewohnte Umgebung, der Routineverlust und die Ungewissheit über den Ablauf des Aufenthaltes werden in Umfragen von Kindern als Gründe für präoperative Ängste angegeben.

Ansetzend an diesen Ergebnissen sollte die präoperative Aufklärung und Vorbereitung durch einen hohen Informationsfluss Bedenken und Ängste minimieren (Coyne 2006). Eine adäquate präoperative Vorbereitung impliziert sowohl eine mögliche pharmakologische Prämedikation als auch aufklärende Gespräche seitens des chirurgischen Teams und der Anästhesie, präoperative klinische Evaluationen sowie den Ausschluss von Kontraindikationen für ein ambulantes Verfahren (Kain et al. 1996). Verschiedene Bedingungen müssen für die ambulante Durchführung einer Operation gegeben sein. Zum einen sollte es sich um eine Operation mit geringem postoperativem Komplikations- und Blutungsrisiko handeln, die ein pflegerisches Handling und eine suffiziente analgetische Therapie durch die Eltern in häuslicher Umgebung erlaubt. Zum anderen muss eben diese Pflege zu Hause durch eine ausreichende Compliance und das nötige kognitive Verständnis gewährleistet sein. Der Bundesverband für Ambulantes Operieren e.V. empfiehlt ein Lebensalter von mindestens drei Monaten als Bedingung für eine ambulante Operation (BAO). Die chirurgische Vorbesprechung in der ambulanten Sprechstunde sollte möglichst mit dem anästhesiologischen Aufklärungsgespräch verbunden werden. Der Ablauf des Operationstages, die Operation selbst, mögliche auftretende Komplikationen und deren Handhabung sowie die Informationen über eine eventuelle Prämedikation sollten die Inhalte der Gespräche darstellen (Stark und Steffens 2006).

Sartori et al. (2015) haben mit Hilfe von Elternbefragungen Aspekte herauskristallisiert, die Bestandteil der präoperativen Vorbereitung sein sollten. 80\% aller Eltern wünschten hier detaillierte Informationen und Beschreibungen bezüglich der Örtlichkeiten (Wartezimmer, Aufwachraum und Operationssaal), der Nüchterperiode prä- und gegebenenfalls postoperativ, der anästhesiologischen Versorgung inklusive Narkoseeinleitung und Anlage der peripheren Venenverweilkanüle, der Medikation und operativer Komplikationen sowie der Erholung zu Hause und Möglichkeiten der Angstreduktion. Das Aufklärungsgespräch sollte bevorzugt im zeitlichen Intervall zum Operationstag stattfinden, sodass ausreichend Zeit für Rückfragen und die Vorbereitung der Eltern und Kinder auf diesen Tag vorhanden ist (Sartori et al. 2015).

In vielen Fällen wird den Kindern vor der Narkoseeinleitung ein kurzwirksames Benzodiazipin, zum Beispiel Midazolam, verabreicht. Dies wirkt nachweislich anxiolytisch und sollte in der Anästhesiesprechstunde präoperativ angeboten werden (Marechal et al. 2017). Die Anwesenheit der Eltern während der anästhesiologischen Einleitung wird 
kontrovers diskutiert. Diverse Studien belegen, dass das Beisein der Eltern keine signifikante Angstreduktion bei den Kindern hervorruft (Kain et al. 2000; Messeri et al. 2004). Durch das Zugegensein der Eltern während der Narkoseeinleitung werden in jedem Falle die Transparenz der präoperativen Abläufe sowie das Vertrauen in die Abteilung gesteigert (Machotta 2014).

\subsubsection{Anästhesie im ambulanten Setting}

Die historische Entwicklung der ambulanten Anästhesie ist das Resultat eines zunehmend sicheren perioperativen Managements, einer multimodalen Schmerzkontrolle, sozioökonomischer Faktoren und einer adäquaten Therapie der anästhesiologischen Nebenwirkungen.

Mitte des 19. Jahrhunderts wurden erstmals Zahnoperationen mithilfe von Ether und wenig später mit Lachgas durchgeführt. Mit der ersten erfolgreichen öffentlich durchgeführten Anästhesie (Etherinhalationsnarkose) am 16. Oktober 1846 in Massachusetts legte William Thomas Green Morton den Grundstein für die Entwicklung der modernen Anästhesie. Ein weiterer Pionier der Chirurgie, J. H. Nicholl, führte bis Anfang des 20. Jahrhunderts mehrere Tausend ambulante Operationen an Kindern durch. J. H. Nicoll diskutierte aufgrund seiner chirurgischen Erfahrungen erstmals Vorteile der postoperativen Genesung zu Hause sowie die Verminderung der nosokomialen Infektionsrate durch eine Reduzierung der stationären Aufenthalte. Im Laufe der Zeit entwickelten sich nicht nur die technischen Möglichkeiten der ambulanten Anästhesie im Sinne einer verbesserten Atemwegssicherung und hochwertigen Anästhesiegeräten weiter, sondern auch die Pharmakologie wurde zusehends fortschrittlicher. Neue Möglichkeiten der Intubations- und Inhalationsnarkosen, der erfolgreichen Schmerzreduktion, der Muskelrelaxation und Regionalanästhesien begünstigten einen stetigen Fortschritt der ambulanten Anästhesie (Urman und Desai 2012). Auch das anästhesiologische perioperative Management gewann zunehmend an Bedeutung. In den späten 70er Jahren wurde im deutschsprachigen Raum die Anästhesieambulanz, oder auch Anästhesiesprechstunde, mit dem Ziel die Versorgung der Patienten durch die Anästhesie zu optimieren, eingeführt. Ein besonderes Augenmerk wird hier auf die präoperative medikamentöse Versorgung, die Evaluation möglicher Risikofaktoren und die Reduktion von Komplikationen und Kosten gelegt. Die intensivierte Betreuung durch die Anästhesisten senkt laut Forschungsliteratur signifikant die Ängste und Sorgen der Patienten bezüglich der bevorstehenden Operation (Dick 1997).

Viele verschiedene Anästhesieverfahren finden im ambulanten Setting Verwendung. Es wird die Allgemeinanästhesie, in Form einer Inhalations- oder intravenösen Narkose, von der Regionalanästhesie unterschieden. Beide Formen können auch kombiniert werden (Ortiz et al. 2014). Die Anwendung allgemeiner intravenöser Narkosen ist mit der Weiterentwicklung gut steuerbarer Hypnotika und Analgetika in der ambulanten Kinderchirurgie häufiger 
geworden. Hier wird als Hypnotikum meist Propofol, Midazolam, Thiopental oder Ketamine verwendet. Eine adäquate Analgesie wird mit Hilfe von Opioiden erreicht (Mani und Morton 2010). Intravenöse Narkosen werden teilweise mit inhalativen Narkosen kombiniert. Ist dies nicht der Fall spricht man von einer total intravenösen Anästhesie, im Folgenden TIVA genannt. Aufgrund der allgemeinen Verfügbarkeit kurzwirksamer Hypnotika und Analgetika ist die TIVA ein gern gewähltes Verfahren im ambulanten Setting (Pandit und Green 1994). Die Allgemeinänasthesie kann auch in Form einer reinen Inhalationsnarkose mit volatilen Anästhetika durchgeführt werden. In der Kinderchirurgie sind häufig verwendete Substanzen Sevofluran, Desfluran, Isofluran, Enfluran, Halothan und Lachgas (Ortiz et al. 2014).

Die Regionalanästhesie gilt in der Kinderchirurgie weniger als alternatives Narkoseverfahren zur Allgemeinanästhesie, sondern dient vielmehr der zusätzlichen peri- und postoperativen Schmerzreduktion. Um eine zu starke Stresssituation für die Kinder zu vermeiden, aber auch aufgrund mangelnder Compliance wird die Regionalanästhesie fast ausschließlich mit einer flachen Allgemeinanästhesie kombiniert. Ein positiver Aspekt der Regionalanästhesie ist das schmerzlose zügige Erwachen nach der Operation. Die Kinder können ohne anästhesiologische Bedenken wenige Stunden nach der Operation schmerzfrei in die häusliche Umgebung entlassen werden (Reich und Brinkmann 2004).

Jeder medizinische Eingriff kann unerwünscht Nebenwirkungen nach sich ziehen. Nach einer Narkose klagen die Patienten häufig über postoperative Übelkeit, Erbrechen (postoperative nausea and vomiting $=$ PONV) und Schmerzen (Oddby et al. 2001). Eine prophylaktische Antiemetikagabe kann helfen PONV zu verhindern und so die Gefahr einer prolongierten Hospitalisation, Dehydratation oder einer Nachblutung durch eine intraabdominale Druckerhöhung beim Erbrechen, zu reduzieren. Mittel der Wahl sind in der Kinderchirurgie neben 5-Hydroxytryptamin- Antagonisten wie Ondansetron und Tropisetron, auch Glukokortikoide wie das Dexamethason. Auch hier zeigt sich ein weiterer Vorteil der Regionalanästhesie, die zusätzlich das postoperative PONV- und auch das Schmerzrisiko deutlich vermindert und so der alleinigen Allgemeinanästhesie überlegen ist (Kovac 2007).

\subsubsection{Schmerztherapie}

Schmerzen gehören zu den wesentlichen postoperativen Komplikationen. Im ambulanten kinderchirurgischen Setting wird die Verantwortung der Schmerzreduktion auf die Eltern der Kinder übertragen. Eine in Finnland durchgeführte Studie hat gezeigt, dass Eltern die Schmerzen ihrer Kinder postoperativ falsch einschätzten und nicht im vorhandenen Ausmaß erwartet haben. In einigen Fällen geben die Eltern laut verschiedener Publikationen die verschriebene Schmerzmedikation nicht in ausreichender Dosierung (Kankkunen et al. 2002). Die Ursachen dieser Untertherapie sind nicht genau evaluiert. Möglicherweise besteht die Problematik in der Identifikation der Schmerzen. Eltern geben in verschiedenen aktuellen 
Befragungen an, dass ihre Kinder oft kein typisches schmerzgeplagtes Verhalten an den Tag legen. Diese Schwierigkeit die Schmerzen der Kinder objektiv einzuschätzen, macht es dem medizinischen Personal zur Aufgabe, die Eltern der Kinder adäquat vorzubereiten und mit Optionen zur Schmerzreduktion auszustatten (Finley et al. 2003). Weiterhin lässt die Wirkung einer perioperativ durchgeführten Regionalanästhesie erst nach einem gewissen Zeitraum nach. Die schmerzfreie Periode zu Hause verhindert gegebenenfalls eine zusätzliche Medikation. Die Kinder entwickeln womöglich bei fehlender Schmerzmittelgabe erst im Verlauf starke Schmerzen. Dieser Umstand ist auch für die Eltern ein erheblicher Stressfaktor (Jonas 2003).

Eine angemessene perioperative Schmerztherapie sollte folglich die intraoperativ durchgeführte Regionalanästhesie, aber auch die additive systemische Schmerztherapie zu Hause und während der postoperativen Überwachungszeit beinhalten. Zusätzlich zur pharmakologischen Therapie spielt bei Kindern die psychologische Betreuung im Sinne einer aufmerksamen, liebevollen Pflege eine wichtige Rolle. Pharmakologische Substanzen, die in der ambulanten Kinderchirurgie häufig und gern verwendet werden, sind unter anderem Paracetamol und NSAIDs (Nonsteroidal anti-inflammatoric drugs) wie Ibuprofen (Russell et al. 2013). Auch Opioide kommen in der Kinderchirurgie zur Anwendung. Um mögliche Nebenwirkungen der Opioide, wie Übelkeit, Erbrechen, Atemdepression und eine Darmatonie zu vermeiden, werden regelmäßig intraoperativ Lokalanästhetika verwendet. Vor allem schwerwiegende Komplikationen, wie die Atemdepression werden so vermieden und somit eine ausreichende Analgesie gewährleistet. Zudem ist die Applikation von Lokalanästhetika der systemischen Opioidgabe überlegen, da sie speziell die afferenten Schmerzbahnen lokal und direkt hemmt. Zwei gängige Methoden der Regionalanästhesie werden in der ambulanten urologischen Kinderchirurgie verwendet: Zum einen die zentrale Kaudalanästhesie und zum anderen die periphere Nervenblockade in Form des Peniswurzelblocks oder der Blockade des N. Ilioinguinalis (Reich und Brinkmann 2004).

\subsubsection{Kaudalanästhesie}

Die Kaudalanästhesie gehört zu den standardmäßig durchgeführten Regionalanästhesien, die am häufigsten in der Kinderchirurgie verwendet werden. Sie kommt vor allem bei subumbilikalen Operationen, wie zum Beispiel der Orchidopexie, zur Anwendung. Diverse Studien belegen, dass es sich hier um ein komplikationsarmes Verfahren handelt. Laut Forschungsliteratur beträgt die Komplikationsrate lediglich 1,9\%, wobei dieser Wert auch falsch platzierte Kaudalblöcke und Versagen der Anästhesie inkludiert (Deer et al. 2016). Die Single-shot-Injektion, also der Kaudalblock ohne Katheter Einlage, repräsentiert mit einer Anwendungshäufigkeit von $80 \%$ die häufigste neuronale Blockade der ambulanten Kinderchirurgie (Stein et al. 2017). Hierbei wird in Seitenlage unterhalb des Conus medullaris durch den Hiatus sacralis unter Perforation der Membrana sakrokokkygea das Lokalanästhetikum (meist Bupivacain oder Rupivacain) in den sakralen Epiduralraum injiziert). Der Hiatus sacralis ist über die Identifikation zweier anatomischer Leitstrukturen, 
die beiden Spinae posteriores superiores, leichter aufzufinden (siehe Abb. 2). Operationen, die mehrere Stunden dauern, erfordern eine ausgedehntere Regionalanästhesie und machen in einigen Fällen die Anlage eines Kaudalkatheters nötig. Dieser kann bis zu 24 Stunden im Körper belassen werden, bevor das Infektionsrisiko signifikant ansteigt. Eine optimale Analgosedierung wird durch eine zeitige Anlage der Kaudalanästhesie gleich nach Einleitung der Allgemeinanästhesie erreicht. Bei korrekter Dosierung der Lokalanästhetika ist perioperativ häufig keine weitere Analgetikagabe erforderlich. Beschriebene Komplikationen sind unter anderem die Duraperforation, Gefäßrupturen und totale Spinalanästhesien. Hohe Erfolgsraten bei selten auftretenden Komplikationen (0,7/1000 Anästhesien verlaufen laut prospektiver Untersuchungen komplikationsbelastet) zeigen, dass es sich bei der Kaudalanästhesie um ein sicheres Verfahren handelt (Reich und Brinkmann 2004).

\subsubsection{Ilioinguinalisblockade}

Bei einseitigen urologischen Eingriffen kann eine zentrale Kaudalanästhesie durch die gezielte Infiltration und Blockade des sensiblen N. ilioinguinalis und des N. iliohypogastricus umgangen werden. Diese Form der Regionalanästhesie kommt häufig bei der operativen Versorgung von Leistenhernien oder bei der Orchidopexie zum Einsatz. Die sensiblen Nerven verlaufen etwa auf Höhe des Leistenbandes zwischen dem Musculus obliquus internus abdominis und dem Musculus transversus abdominis medial zum Leistenkanal und könnten hier exakt mit einem Lokalanästhetikum infiltriert werden (Reich und Brinkmann 2004).

\subsubsection{Peniswurzelblock}

Der Peniswurzelblock ist als wirkungsvolle Methode zur Schmerzausschaltung Mittel der Wahl bei urologischen Operationen am distalen Penis. Seine suffiziente analgetische Wirkung ist meist ausreichend, sodass im besten Fall keine zusätzliche perioperative Analgetikagabe notwendig ist. Unter einer ausreichenden Allgemeinanästhesie wird paramedian der Mittellinie in den subpubischen Raum ein Lokalanästhetikum in hoher Konzentration bei geringem Volumen infiltriert. Da es sich bei dem Nervus dorsalis penis um einen rein sensiblen Nerv handelt, kann auf eine vorhergehende Stimulation verzichtet werden. Der Peniswurzelblock gilt laut verschiedener Studien als eine sehr sichere, komplikationsarme Regionalanästhesie, die bei Kindern problemlos durchgeführt werden kann (Reich und Brinkmann 2004).

\subsection{Krankheitsbilder und Operationstechniken}

Im folgenden Kapitel werden die bei den Kindern prozentual am häufigsten vertretenen Krankheitsbilder und die durchgeführten Operationstechniken thematisiert. 


\subsubsection{Hodendystopien}

Der Begriff Hodendystopie bezeichnet den nicht im Skrotum gelegenen Hoden. Diese Fehlbildung gehört zu den häufigsten angeborenen Anomalien des Jungen. Die Prävalenz bei reifen Neugeborenen beträgt ungefähr 3\% (Kollin et al. 2007). Je nach Lage des Hodens wird die Hodendystopie in verschiedene Formen unterteilt. Man differenziert den Pendelhoden und den Gleithoden von der Hodenretention (Bauch- und Leistenhoden) und der Hodenektopie. Der Pendelhoden beschreibt einen normal ins Skrotum deszendierten Hoden, der zeitweilig durch zum Beispiel einen sehr empfindlichen Kremasterreflex an das obere Scrotalfach oder sogar in den Inguinalkanal wandert.

Befindet sich der Testis überwiegend inguinal und ist nur durch manuelle Reposition ins Skrotum verlagerbar, schnellt aber direkt zurück, spricht man von einem Gleithoden.

Die Hodenretention, oder auch Maldescensus testis, bezeichnet den unvollständigen Descencus des Hodens, der in diesem Fall im Leistenkanal verbleibt. Je nach Lage des Hodens spricht man von einer inguinalen oder abdominellen Retention. Hier befindet sich der Hoden noch in dem Leistenkanal, bzw. der Abdominalhöhle und ist weder tast- noch sichtbar (Sökeland und Rübben 2007).

Folgen einer Hodendystopie können unter anderem die gestörte Fertilität und ein erhöhtes Risiko für Hodentumore sein. Ab dem zweiten Lebensjahr kann bei jungen Patienten mit Hodendystopie eine deutliche Verringerung der Spermatogonienanzahl und des Hodenvolumens, eine veränderte Qualität der Spermien sowie verdicktes peritubuläres Bindegewebe festgestellt werden. Vor diesem Hintergrund ist eine zeitige Korrektur der Hodenlage empfehlenswert (Hrivatakis et al. 2014).

Eine weitere prägnante Komplikation des dystopen Hodens ist das oben genannte Risiko für maligne Hodentumore, welches bei einer Hodenfehlanlage ungefähr fünf Mal höher ist als bei einem orthotopen Hoden. Besonders gefährdet sind die abdominell gelegenen Hoden, hier ist das Risiko der Entartung nochmals um das Vierfache gesteigert. Das erhöhte Risikoprofil der Entartung ergibt sich aus verschiedenen Faktoren. Unter anderem die Atrophie und Dysgenesie, also eine Verkleinerung beziehungsweise Fehlbildung des Hodens sowie hormonelle Funktionsstörungen können Ursache maligner Entartungen sein.

Fehlbildungen des Hodens gehen gehäuft mit weiteren Anomalien einher, sodass eine sonographische Kontrolle der Niere sowie der ableitenden Harnwege zusätzlich zur Hodeninspektion und Ultraschallkontrolle des Adomens erfolgen sollte. Außerdem wird empfohlen bei einer erforderlichen Orchidopexie intraoperativ den Processus vaginalis mit zu inspizieren, da dieser bei 50\% der Patienten mit Hodendystopie noch nicht obliteriert ist und dann in der gleichen Sitzung unterbunden werden kann (Sökeland und Rübben 2007).

\section{Orchidopexie}

Hat sich der dystope Hoden nicht im frühen Kindesalter spontan in das Skrotum verlagert, ist eine chirurgische Reposition notwendig. Die Orchidopexie bezeichnet das spannungsfreie 
Rückverlagern und Fixieren des Testis im Skrotum. Es gibt verschiedene Operationstechniken, die abhängig von der Lage des dystopen Testis gewählt werden. Ausschlaggebend ist hier die manuelle Reposition des Hodens in das Skrotum. Ist es möglich den Hoden bis in das Skrotum zu mobilisieren, kann er über einen skrotalen Zugangsweg direkt fixiert werden. Ist der Hoden entweder nicht verlegbar oder bleiben Rücklagerungsversuche frustran, wird zusätzlich ein inguinaler Hautschnitt benötigt, um den Hoden zu mobilisieren (Misra et al. 1997). Beide Operationsverfahren kamen in dieser Studie zum Einsatz und wurden jeweils in Allgemeinnarkose und meist zusätzlich in regionaler Kaudalanästhesie durchgeführt.

Bei dem inguinalen Zugangsweg wird im ersten Schritt über eine inguinale Inzision der Leistenkanal eröffnet und nach Identifikation des Funikulus spermatikus die Faszie und Muskelfasern des Muskulus cremaster durchtrennt. Jetzt kann die Mobilisation zum Anulus inguinalis profundus erfolgen. Hier wird unter Schonung der Gefäße und des Ductus deferens die Faszia spermatica interna abgelöst und der Hoden mit Hilfe einer Klemme im Inguinalkanal fixiert (Hutcheson et al. 2000).

Im zweiten Schritt wird über einen Hautschnitt eine Tasche zwischen Kutis und Tunica Dartos am tiefsten Punkt des Skrotums präpariert. Eine weitere Klemme, die über eine kleine Eröffnung dieser Dartostasche in die inguinale Inzision eingeführt wird, greift den Hoden und zieht ihn nach kaudal in die hergerichtete subkutane Tasche. Anschließend erfolgt die Fixierung des Testis mit zwei Nähten. Im letzten Schritt wird die Haut des Skrotums und des inguinalen Zugangs mit einer resorbierbaren Naht verschlossen (Mohey et al. 2017).

Ist der Hoden im Skrotum zu tasten oder spannungsfrei in das Skrotum zu mobilisieren, kann ein weniger invasives Verfahren mit nur einem Schnitt gewählt werden. Hierbei wird eine transversale Inzision am unteren Skrotum durchgeführt. Auch bei dieser Operationstechnik wird eine subkutane Dartostasche präpariert und inzidiert. Durch Druck auf den Inguinalkanal wird der Testis durch die Eröffnung dargestellt und mit zwei Fingern fixiert. Es folgt die Entfernung der Hodenhüllen sowie die Durchtrennung des Muskulus cremaster und des Processus unter Schonung des Ductus deferens und der Gefäßstrukturen. Wichtig ist vor endgültiger Fixierung des Hodens einen offenen Processus vaginalis auszuschließen. Ist dieser obliteriert, kann der Hoden spannungsfrei in der vorbereiteten Tasche befestigt werden (Mohey et al. 2017).

\subsubsection{Phimose}

Die Phimose bezeichnet eine Verengung der Vorhaut, die ihr Zurückstreifen erschwert oder unmöglich macht. Physiologisch ist das Zurückziehen der Vorhaut nicht von Geburt an möglich. Beim Neugeborenen sind die Glans und das Präputium durch ein Plattenepithelseptum getrennt. Der präputiale Raum entsteht erst im Laufe der Entwicklung durch einen Abschilferungsprozess der Epithelzellen dieses Septums. Erst mit ungefähr zwei Jahren ist bei $80 \%$ der Jungen, mit drei Jahren bei $90 \%$ der Jungen, die Vorhaut komplett 
reponierbar. Eine unbehandelte pathologische Phimose kann verschiedene Komplikationen zu Folge haben. Zum einen kann es durch die enge Präputialöffnung zu einer Ballonierung der Vorhaut während der Miktion mit nachfolgender Harnwegsobstruktion kommen, zum anderen können durch den Retraktionsversuch Fissuren und Rhagaden entstehen. Weitere Komplikationen sind die Smegmaretention (ein Transsudat mit abgeschilferten Epithelzellen), die Balanitis (Eichelentzündung) und die Paraphimose. Die Paraphimose bezeichnet eine Einklemmung des Präputiums im Sulcus Coronarius, proximal der Glans penis. Diese Einklemmung führt schnell zu einer Zirkulationsstörung, vor allem der venösen- und der Lymphgefäße, welche eine ödematöse Schwellung der Glans und des Präputiums sowie starke Schmerzen verursacht. Um zu verhindern, dass die arterielle Blutzufuhr durch das entstandene Ödem ebenfalls unterbrochen wird und es zu einer nekrotischen Gangrän kommt, ist eine schnelle Reposition, wenn möglich manuell oder operativ durch Inzision, anzustreben (Hautmann und Huland 2006).

Es gibt verschiedene Möglichkeiten eine Phimose zu behandeln. Vor der operativen Korrektur wird meist ein konservativer Versuch mit Steroidsalben angewendet, der häufig bereits zum gewünschten Erfolg führt. Ist der konservative Therapieansatz aufgrund einer therapierefraktären Phimose oder auch durch mangelnder Compliance erfolglos, besteht die Indikation für eine Operation. In der Literatur werden diverse Varianten der Phimosen Operation beschrieben, klassischerweise wird aber heutzutage meist die komplette Zirkumzision durchgeführt (Dewan et al. 1996).

\section{Zirkumzision}

Besteht bei einem Jungen nach dem dritten Lebensjahr noch immer eine Phimose, oder macht eine bestehende Phimose vorher bereits Komplikationen, ist die Indikation zur Zirkumzision, umgangssprachlich als Beschneidung bezeichnet, gestellt. Der chirurgische Eingriff erfolgt in Allgemeinnarkose. Zusätzlich kann eine regionale Anästhesie in Form eines Penisblocks oder einer Kaudalanästhesie die Schmerzen reduzieren. Im ersten Schritt erfolgen bei allen Techniken die Präputiolyse, also das Lösen der verklebten Vorhaut sowie gegebenenfalls eine Inzision des Frenulums, eine Schleimhautfalte an der Unterseite der Glans. Anschließend wird die Vorhaut angespannt und distal abgetrennt mit anschließender Nachresektion des inneren und äußeren Vorhautblattes und darauffolgender Blutstillung. Die Wunde wird abschließend mit synthetischen resorbierbaren Einzelknopfnähten verschlossen und mit einem Pflasterverband versorgt (Deindl 2016).

\subsubsection{Leistenhernien}

Der Leistenkanal verläuft oberhalb des Leistenbandes und enthält neben Nerven, Arterien und Lymphgefäßen beim Mann zusätzlich den Funiculus spermaticus sowie bei der Frau das Ligamentum teres uteri. Er wird durch verschiedene Strukturen begrenzt. Das kraniale Dach bilden der Musculus obliquus inferior sowie der Musculus transversus abdominalis. Die Faszia transversalis und das Ligamentum interfoveolare begrenzen den Leistenkanal nach 
dorsal, die Aponeurose des Musculus obliquus externus begrenzt ihn nach ventral. Den kaudalen Schenkel bildet das Ligamentum inguinale. Die Öffnungen des Leistenkanals werden als innerer und äußerer Leistenring, oder auch Anulus inguinalis profundus und superfizialis bezeichnet (OA 2014). Leistenhernien bestehen aus einem Bruchsack mit dem Bruchinhalt (Eingeweideanteil), sowie einer Bruchpforte. Man unterscheidet die direkte mediale Leistenhernie von der indirekten lateralen Hernie anhand ihrer Durchtrittsstelle durch die Bauchwand. Bei Kindern ist die indirekte laterale Leistenhernie deutlich häufiger vertreten. Hier verläuft die Hernie vom inneren bis zum äußeren Leistenring. Ursächlich ist hier entweder eine fehlende Obliteration des Processus vaginalis oder aber eine Aufweitung des inneren Leistenrings mit folgender Vorwölbung des Peritoneums in den Leistenkanal (Müller et al. 2016; 2017).

\section{Herniotomie}

Aufgrund der Gefahr der Inkarzeration der Hernie (bei Kindern und Jugendlichen zwischen sechs und 18 Prozent) besteht schon bei Diagnosestellung eine Operationsindikation (Esposito et al. 2017). Die Herniotomie bei Kindern unterscheidet sich deutlich von der Hernienversorgung bei Erwachsenen. Bei Kindern wird auf die Einlage von Fremdmaterial ganz verzichtet, ein Verschluss des Bruches mit Nähten ist ausreichend. Der Eingriff wird üblicherweise in Allgemeinnarkose durchgeführt. Im ersten Schritt erfolgt eine transverse Inzision in der Leiste des Kindes (Potts et al. 1950). Anschließend wird der Anulus inguinalis superfizialis dargestellt und von dort aus wird der Leistenkanal eröffnet (Operationstechnik nach Fergusson). Der Bruchsack mit seinem Bruchinhalt wird vom Funikulus spermatikus bzw. beim Mädchen vom Ligamentum teres uteri separiert und bis zum Anulus inguinalis profundus präpariert. Hier erfolgt die Eröffnung des Bruchsackes und nach Rückverlagerung der vorgefallenen Organe die Fixierung mit Nahtmaterial an seiner Basis. Der Überschuss wird reseziert und anschließend wird der Leistenkanal wieder rekonstruiert. Der Hautverschluss erfolgt mit resorbierbarem Nahtmaterial oder Gewebekleber (Göbel und Stuhldreier 2008).

Alternativ gewinnt seit einigen Jahren die laparoskopische Hernienversorgung bei Kindern an Bedeutung. Verschiedene Studien belegen, dass der minimal invasive Zugang über das Laparoskop nicht nur die Operationszeit und die postoperativen Schmerzen vermindern soll, sondern auch ein besseres kosmetisches Ergebnis erzielt (Liu et al. 2014). Es existieren viele verschiedene laparoskopische Techniken zur Herniotomie. Zusammenfassend lassen sich die extrakorpulären-, von den intrakorpulären Verfahren abgrenzen (Esposito et al. 2017). Diese Differenzierung bezieht sich auf die Art und Weise, mit der der Procesuss vaginalis ligiert wird. Klassischerweise werden drei Trokare verwendet, einen für die Optik und zwei für die chirurgischen Instrumente. Nach Einsetzen der Trokare wird im ersten Schritt die Öffnung des Processus vaginalis überprüft. Anschließend erfolgt die zirkumferente Inzision des Bruchsackes auf Höhe des Anulus inguinalis profundus ähnlich wie bei den offenen Operationsverfahren (Niyogi et al. 2010). Die Bruchlücke wird im Anschluss mit nicht 
resorbierbarem Nahtmaterial verschlossen. Eine Kontrolle des kontralateralen Processus vaginalis ist mit dem Laparoskop gut möglich. Ein offener Processus vaginalis der Gegenseite kann so intraoperativ identifiziert und gegebenenfalls während desselben Eingriffs mitversorgt werden (Esposito et al. 2009).

\subsection{Studienziel}

Wie bereits in Kapitel 1.1 beschrieben, beeinflussen viele verschiedene Aspekte die Zufriedenheit und die erlebte Sicherheit der Eltern von ambulant operierten Kindern.

Das ambulante Setting der Operation sollte sich nach diesen Faktoren richten, um diesen anstrengenden und stressigen Tag vor allem für die Kinder, aber auch für ihre Eltern so erträglich wie nur eben möglich zu gestalten. Es gilt schlechte Erfahrungen des Kindes mit der medizinischen Umgebung und der Behandlung durch das medizinische Personal zu vermeiden und die Eltern auf die Versorgung des Kindes vor und nach der Operation zu Hause bestmöglich vorzubereiten. Hierzu ist es nötig, den Ablauf des Operationstages im Vorfeld so transparent wie möglich zu gestalten und die Eltern über gewissen Maßnahmen zu Hause, wie zum Beispiel die Gabe von Schmerzmedikamenten, ausführlich aufzuklären und weitere strukturelle Rahmenbedingungen wie weitere Arzttermine festzulegen.

Welche Art und Weise der Aufklärung die höchstmögliche Sicherheit der Eltern zu Hause bedingt, wollten wir mit der vorliegenden Arbeit evaluieren. Hierzu beschäftigten wir uns mit dem Einfluss eines neu entwickelten Behandlungskonzeptes auf die erlebten prä- und postoperativen Stunden sowie auf die postoperativen Schwierigkeiten zu Hause. Dieses Behandlungskonzept beinhaltet eine schriftliche Dokumentation der relevantesten Informationen in Form eines Informationsblattes für die weitere medizinische Versorgung durch die Eltern zu Hause. Es sollen genaue zeitliche Angaben bzgl. des nächsten Kinderarztbesuches und Pflasterwechsels und eine Empfehlung hinsichtlich der Schmerztherapie und des Verhaltens zu Hause (Baden, Duschen, Sport/Toben) vermittelt werden. Außerdem wurde ein neuer Informationsbogen formuliert, den die Eltern präoperativ in der ambulanten Sprechstunde erhielten. Er beinhaltet wichtige organisatorische Daten (Anmeldezeit- und Ort präoperativ im Krankenhaus, Kontaktdaten für eventuelle Rückfragen, Zeiten der Nüchterperiode) sowie eine Wegbeschreibung zur präoperativen Anästhesiesprechstunde und zur Anmeldung im Zentral- OP am Tag der Operation.

Ziel dieses Behandlungskonzeptes sollte die Optimierung des perioperativen Managements sein. Durch die terminierten Angaben zur Schmerzmittelgabe wollten wir die postoperativen Schmerzen der Kinder minimieren und die Eltern entlasten, indem wir Ihnen die Entscheidung ein Analgetikum zu geben, abnahmen.

Von der zusätzlichen schriftlichen Dokumentation der ärztlichen Empfehlung für die postoperative ambulante Nachsorge erhofften wir uns eine transparentere und nachhaltigere 
Aufklärung, die mit einer erhöhten Elternzufriedenheit mit dem perioperativen Management und einer verstärkten Festigung der vermittelten Information einhergehen sollte. Ängste der Eltern bezüglich des Umgangs mit dem Kind sollten so reduziert und die Versorgung des Kindes zu Hause erleichtert werden.

Die mitgegeben Kontaktdaten bieten sowohl den Eltern als auch dem Kinderarzt, der die ambulante Nachsorge durchführt, die Möglichkeit der Korrespondenz bezüglich fachlicher Rückfragen oder dient als Ansprechpartner bei Notfällen.

Ein weiteres Ziel des Behandlungskonzeptes war die Optimierung der Abläufe am Tag der Operation. Die Informationsbögen mit den organisatorischen Daten sollten nicht nur das Zeitmanagement verbessern, Wartezeiten reduzieren und infolgedessen den Tag für die Kinder und auch für ihre Eltern erheblich erleichtern, sondern auch die Operationsplanung für das medizinische Personal vereinfachen. Zusätzlich galt es die Wahrscheinlichkeit für operative Voraussetzungen, wie zum Beispiel die ausreichende präoperative Nüchternperiode, auch im Sinne eines adäquaten Zeitmanagements zu erhöhen.

Insgesamt erhofften wir uns von dem neuen Behandlungsplan eine Reduktion der präoperativen Sorgen und Ängste seitens der Eltern und dementsprechend auch seitens der Kinder. Die Angst vor der ungewohnten Umgebung, vor möglichen Schmerzen und die starke Sorge um das gesundheitliche Wohl des Kindes sollte möglichst verringert werden. Das Ziel war hier mit Hilfe vorgegebener Strukturen und schriftlichen Informationen mögliche Unsicherheiten der Eltern zu beseitigen und sie in die postoperative Versorgung des Kindes zu integrieren und bestmöglich vorzubereiten. 


\section{Material und Methoden}

\subsection{Studienablauf}

Im Rahmen der Studie wurden im Zeitraum von Oktober 2015 bis September 2016 bei hundert Patienten im Alter von eins bis 14 Jahren operationstechnische Daten, postoperative Befunde wie Schmerzen, Übelkeit, Erbrechen und Nachblutungen sowie die Zufriedenheit der Eltern bzw. der Erziehungsberechtigten mit dem perioperativen Management mit Hilfe von zwei Fragebögen evaluiert. Unterteilt wurden die hundert Patienten in zwei Kollektive, die jeweils fünfzig Patienten umfassen. Bei den ersten fünfzig Patienten wurden die Daten vor der Änderung des Behandlungskonzeptes aufgenommen, weitere fünfzig Patienten wurden erst nach Einführung des neuen Konzeptes kontaktiert und befragt. Die Idee des angesprochenen neuen Behandlungskonzeptes war es, die Schmerzmedikation der Kinder und weitere therapeutische Maßnahmen (zum Beispiel ein terminlich festgelegter Verbandswechsel und Arztbesuch, der Termin für das Fäden ziehen, genaue Angaben ab wann Sport, Baden, Essen und Trinken wieder normal durchführbar sind) nicht nur mittels mündlicher Absprache anzusetzen, sondern in Form eines zeitlich festgelegten Therapieplans schriftlich $\mathrm{zu}$ übermitteln. Außerdem wurde ein neuer Informationsbogen formuliert, den die Eltern präoperativ in der ambulanten Sprechstunde erhielten. Er beinhaltet wichtige organisatorische Daten (Anmeldezeit- und Ort präoperativ im Krankenhaus, Kontaktdaten für eventuelle Rückfragen, genaue Zeiten der Nüchtern Periode sowie eine Wegbeschreibung zur präoperativen Anästhesiesprechstunde). Außerdem wurde die Empfehlung ausgesprochen am Tag vor der Operation die präzise Einbestellzeit in der Kinderchirurgischen Sprechstunde zu ermitteln und so die Wartezeit bis zur Narkoseeinleitung zu reduzieren.

Die Eltern beider Patientengruppen erhielten den ersten Fragebogen zur Evaluation zusammen mit der Einverständniserklärung in der ambulanten Kinderchirurgiesprechstunde der UMG. Diese Bögen wurden ausgefüllt am Tag der Operation in der Aufnahme abgegeben. Weitere Daten wurden am ersten postoperativen- sowie am 5. postoperativen Tag mittels eines telefonisch durchgeführten Fragebogens erfasst und während des Gesprächs in eine elektronische Datenbank (verwendet wurde hier das Programm Exel von Microsoft) aufgenommen. Befragt wurde ein Elternteil oder ein Erziehungsberechtigter der Kinder. Zusätzlich zu den Fragebögen wurden Informationen aus den elektronischen Akten der Kinder, speziell aus den Anästhesieprotokollen und den Operationsberichten entnommen und ebenfalls in die elektronische Datenbank eingespeist. Folgende Daten wurden auf diese Weise erhoben: die Diagnose, die Art der Operation, eventuelle Voroperationen, die Operationsdauer, das gewählte Anästhesieverfahren inklusive Art der Atemwegssicherung, die eventuelle Anlage eines Blasenverweilkatheters, prä- und intraoperativ verabreichte Medikamente sowie allgemeine Daten des Kindes, wie Geburtsdatum, Geschlecht und Gewicht. 
Die statistische Auswertung des Datensatzes erfolgte mit dem Programm STATISTICA 13.0 German.

Ein Ethikantrag an die Ethikkommission der Universitätsklinik Göttingen lag vor.

\subsubsection{Entwicklung der Fragebögen}

Der Fragenbogen besteht aus verschiedenen Items vom Likert Typ, die mit fünf verschiedenen Antwortmöglichkeiten (trifft gar nicht zu, trifft eher nicht zu, unentschieden, trifft eher zu, trifft völlig zu) beantwortet werden. Drei Fragen werden offen beantwortet, wenige Fragen sind mit Ja oder Nein zu beantworten. Thematisch ist der Fragenkatalog in drei Blöcke untergliedert. Der erste Block bezieht sich auf die Schmerzmedikation und mögliche Komplikationen. Im zweiten Teil des Fragebogens wird näher auf medizinische Aufklärungen und strukturellen Aspekte des Operationstages eingegangen. Der dritte Block beinhaltet Fragen zur perioperativen Zufriedenheit der Eltern, zu der Nachsorge und zur Versorgung durch das medizinische Personal. Jeweils eine originale Version der Fragebögen findet sich in dem Anhang dieser Arbeit.

\subsubsection{Patientenrekrutierung und Kollektiv}

Das Gesamtkollektiv der Studie umfasst hundert Patienten, die in der Allgemein-Viszeralund Kinderchirurgie der Universitätsklinik Göttingen in dem Zeitraum von Oktober 2015 bis zum September 2016 operiert wurden. Einziges Einschlusskriterium war in diesem Falle die Operation, die ambulant erfolgen muss. Ausschlusskriterien waren abgesehen von einem stationären Aufenthalt keine vorhanden. Die Erziehungsberechtigten der Kinder wurden im Rahmen der kinderchirurgischen Sprechstunde über die geplante Studie informiert und um die Teilnahme gebeten. Sie erhielten nach ausführlicher Aufklärung und Zustimmung zur Studienteilnahme jeweils eine Einverständniserklärung und den ersten Fragebogen zur Datenerfassung.

Teilweise sind die Daten der Patienten unvollständig. Eine Beeinträchtigung der Ergebnisse durch die fehlenden Daten ist uns bewusst, trotzdem sahen wir von einem Ausschluss dieser Patienten aus der Studie ab, um ein möglichst großes repräsentatives Patientenkolletiv zu bewahren. In der Ergebnisdarstellung und der Diskussion sind diese Daten entsprechend beschrieben. 


\subsubsection{Datenermittlung und IXSERV}

Sowohl die telefonisch ermittelten Daten als auch die Daten der abgegebenen Fragebögen wurden zunächst in das Programm Excel von Microsoft Office $15{ }^{\circledR}$ eingegeben (Home and Student 2013) und anschließend gesammelt in das Programm Statistica ${ }^{\circledR} 13.0$ zur statistischen Auswertung eingespeist. Die Speicherung dieser Daten erfolgte anonymisiert. Den einzelnen Patienten wurden dafür Fallnummern (fortlaufend von 1- 100 in zeitlicher Abfolge) zugeteilt. Einzig die telefonische Erhebung der Daten erfolgt noch namensgebunden, dokumentiert wurden diese Daten aber nur anhand der Fallnummern. Die Antworten der Studienteilnehmer wurden in codierter Form in den Datensatz übertragen. Die Zuordnungen der Antwortmöglichkeiten zu den Zahlen sind der Tabelle 1 zu entnehmen.

Tabelle 1: Zuordnung von Antwort und codierter Nummer.

\begin{tabular}{|l|l|}
\hline Antwort & Codierte Nummer \\
\hline Ja & 1 \\
\hline Nein & 0 \\
\hline Trifft gar nicht zu & 1 \\
\hline Trifft eher nicht zu & 2 \\
\hline Unentschieden & 3 \\
\hline Trifft eher zu & 4 \\
\hline Trifft völlig zu & 5 \\
\hline
\end{tabular}

Neben allgemeinen patientenspezifischen Daten, wie zum Beispiel dem Alter, Geschlecht und Gewicht wurden unter anderem auch operationstechnische Daten ermittelt. In Anlegung an das Thema dieser Dissertation wurde auf die Parameter der perioperativen Versorgung (Komplikation, Zufriedenheiten, Organisation) ein besonderer Schwerpunkt gelegt.

Das beobachtete Befundspektrum umfasst postoperative Schmerzen, Übelkeit, Erbrechen, Blutungen und Müdigkeit. Zur genaueren Differenzierung wichtiger Parameter des gelungenen perioperativen Managements wurde nicht nur die allgemeine Zufriedenheit der Eltern evaluiert, sondern auch einzelne Organisationspunkte, wie zum Beispiel die Aufklärung über Schmerzen, Blutung und Narkoseverfahren erfasst.

Die Struktur des Operationstages wurde anhand der Wartezeiten (prä- und postoperativ), der Operationszeiten und des Transportes nach Hause beurteilt. Der Wunsch nach einer Kontrolluntersuchung durch den zuweisenden Kinderarzt und die Vermittlung einer 
medizinischen Kontaktperson für eventuelle Rückfragen wurden im Zusammenhang mit der erlebten Sicherheit der Eltern evaluiert.

Für die Ermittlung der operationstechnischen Daten wurde das krankenhausinterne Programm IXSERV verwendet. Die genauen Quellenangaben der erhobenen Daten sind im Folgenden beschrieben (s. Tabelle 2).

Tabelle 2: Quellen der Datenerhebung.

\begin{tabular}{|c|c|}
\hline Art der Daten & Quelle \\
\hline Alter & Aufnahmeprotokoll \\
\hline Geschlecht & Aufnahmeprotokoll \\
\hline Gewicht & Anästhesieprotokoll \\
\hline Diagnose & Operationsbericht \\
\hline \multicolumn{2}{|l|}{ Operationstechnische Daten: } \\
\hline Art des Eingriffs & Operationsbericht \\
\hline Voroperationen & Anästhesieprotokoll \\
\hline OP- Datum & Operationsbericht \\
\hline OP- Dauer & Anästhesieprotokoll \\
\hline \multicolumn{2}{|l|}{ Anästhesiologische Daten: } \\
\hline Verwendete Anästhesieform & Anästhesieprotokoll \\
\hline Art der Atemwegssicherung & Anästhesieprotokoll \\
\hline Medikation prä- und intraoperativ & Anästhesieprotokoll/Pflegebericht \\
\hline Anlage Blasenkatheter & Anästhesieprotokoll \\
\hline Antworten Fragenkatalog & $\begin{array}{l}\text { Telefonische Evaluation und schriftliche } \\
\text { Auswertung der eingereichten Fragebögen }\end{array}$ \\
\hline
\end{tabular}

\subsection{Datenauswertung mit Statistica}

Die Auswertung des Datensatzes erfolgte mit dem Programm Statistica 13.0 German mit der freundlichen Unterstützung des medizinischen statistischen Instituts der Universitätsmedizin Göttingen. Zunächst wurden alle gesammelten Daten in das Programm eingetragen. Im Anschluss erfolgten sowohl die deskriptive Auswertung als auch verschiedene Korrelationen.

Die deskriptive Auswertung umfasste Häufigkeitsverteilungen von allen metrischen, ordinalen und nominalen Daten. Zur optischen Veranschaulichung wurden Box-Plots, Kreisdiagramme, Histogramme und Häufigkeitstabellen verwendet. Mit Hilfe der 
Kreisdiagramme und Histogramme lässt sich die Häufigkeitsverteilung der untersuchten Parameter schematisch darstellen. Die Häufigkeitstabellen präsentieren die Verteilungen der Daten in numerischer Form. Weiterhin wurden für einige quantitative Merkmale der Median, oberes und unteres Quartil und der obere und untere Whisker bestimmt.

Zur Berechnung von Korrelationen zwischen zwei Variablen wurden verschiedene Tests verwendet. Zum Einsatz kamen unter anderem die Spearmans-Rangkorrelation, PearsonChi-Quadrat-Tests, der Kruskal-Wallis Test, der Mann-Whitney-U-Test und der KaplanMeier-Schätzer.

Bei allen Korrelationen gilt ein Zusammenhang als signifikant, wenn die berechnete Fehlerwahrscheinlichkeit $\mathrm{p} \leq 0,05$ (alpha Level) ist.

Der Zusammenhang zwischen der allgemeinen Zufriedenheit der Eltern mit dem perioperativen Management, dem Alter der operierten Kinder sowie der Aufklärung über eine eventuelle Nachblutung, möglicherweise auftretende Schmerzen und deren Management wurde mit der Spearman-Rangkorrelation berechnet. Der hier verwendete Rangkorrelationskoeffizient ist ein parameterfreies Maß. Bei beiden Variablen handelt es sich um ordinale Daten.

Bei Variablen mit linearem Zusammenhang ohne Normalverteilung wurde mit dem ChiQuadrat-Test nach K. Pearson ein Hypothesentest zur Verteilung der Variablen durchgeführt. Beobachtet wurde hier die Komplikation „Schmerzen“, zum einen im Vergleich mit der Zeit bis zur Beschwerdefreiheit, zum anderen mit der erfolgten Anlage einer Kaudalanästhesie.

Der Mann-Whitney-U-Test ist ein nicht parametrischer Homogenitätstest und dient der Signifikanzüberprüfung zweier Verteilungen. Jede postoperativ beobachtete Komplikation wurde mit Hilfe dieses Testverfahrens mit dem Alter der Kinder in Korrelation gesetzt.

Ein ähnliches Testverfahren ist der Kruskal-Wallis-Test. Er überprüft ebenfalls, ob zwei Variablen derselben Grundgesamtheit entspringen, kann jedoch auch angewendet werden, falls mehr als zwei Gruppen untersucht werden. In diesem Falle wurde die Operationszeit oder die Zeit postoperativ und die aufgetretenen Schmerzen bei den Kindern miteinander verglichen.

Der Kaplan-Meier-Schätzer bestimmt die Wahrscheinlichkeit, nach der ein bestimmtes Ereignis in einem gewissen Zeitraum für eine spezifische Variable nicht eintritt. Untersucht wurde hier der Zeitraum bis zum Auftreten der Beschwerdefreiheit in Abhängigkeit von dem angegebenen Schmerzskalenniveau. 


\section{Ergebnisse}

Im Folgenden werden die Ergebnisse der statistischen Auswertung dargestellt. Sowohl die deskriptiven Daten als auch die Korrelationen werden aufgeführt und teilweise anhand verschiedener Abbildungen näher erläutert.

\subsection{Patientenkollektiv}

Das Patientenkollektiv der Studie umfasst insgesamt hundert Patienten ( $\mathrm{n}=100)$. Das männliche Geschlecht überwiegt hier deutlich. 92 Jungen und acht Mädchen wurden im Zeitraum von elf Monaten an der Universitätsklinik in der ambulanten Kinderchirurgie operiert (s. Abbildung 1). Ergab sich postoperativ doch eine Indikation für eine stationäre Aufnahme, wurden die Kinder von der Studie ausgeschlossen.

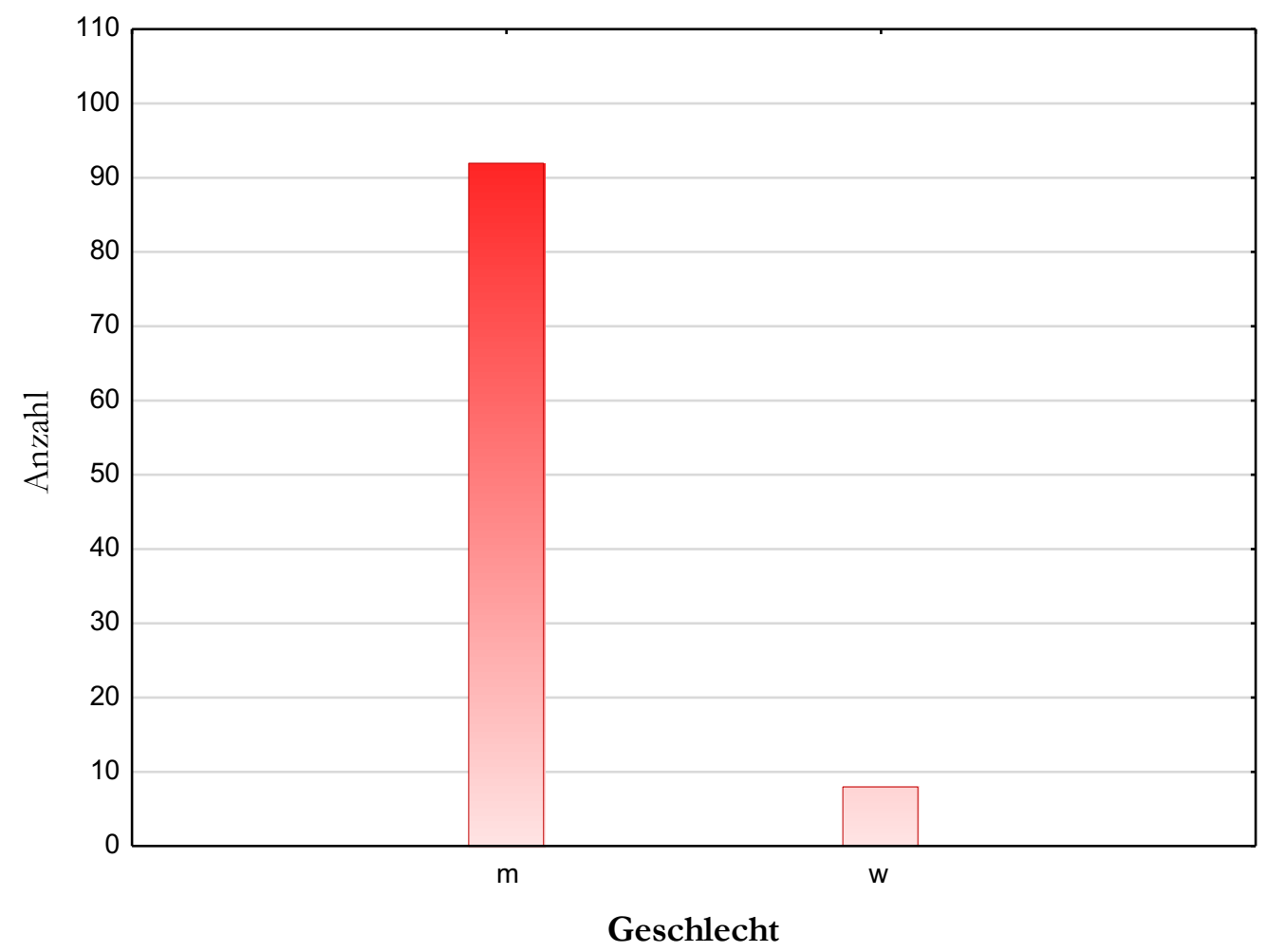

Abbildung 1: Geschlechterverteilung. 
Die teilnehmenden Kinder waren zum Zeitpunkt der durchgeführten Operation alle zwischen elf Monaten und zwölf Jahren alt. 34 Kinder befanden sich in der Altersgruppe der ein bis drei Jährigen, 43 Patienten in der Gruppe der vier bis sechs jährigen, nur zwölf Kinder waren sieben bis neun Jahre alt und elf Kinder waren zehn bis zwölf Jahre alt. Das durchschnittliche Alter der Mädchen betrug $6 \pm$ 0,85 Jahre, das der Jungen 4,8 \pm 0,3 Jahre. Der Median für das Gesamtalter liegt bei 5 (s. Abbildung 2).

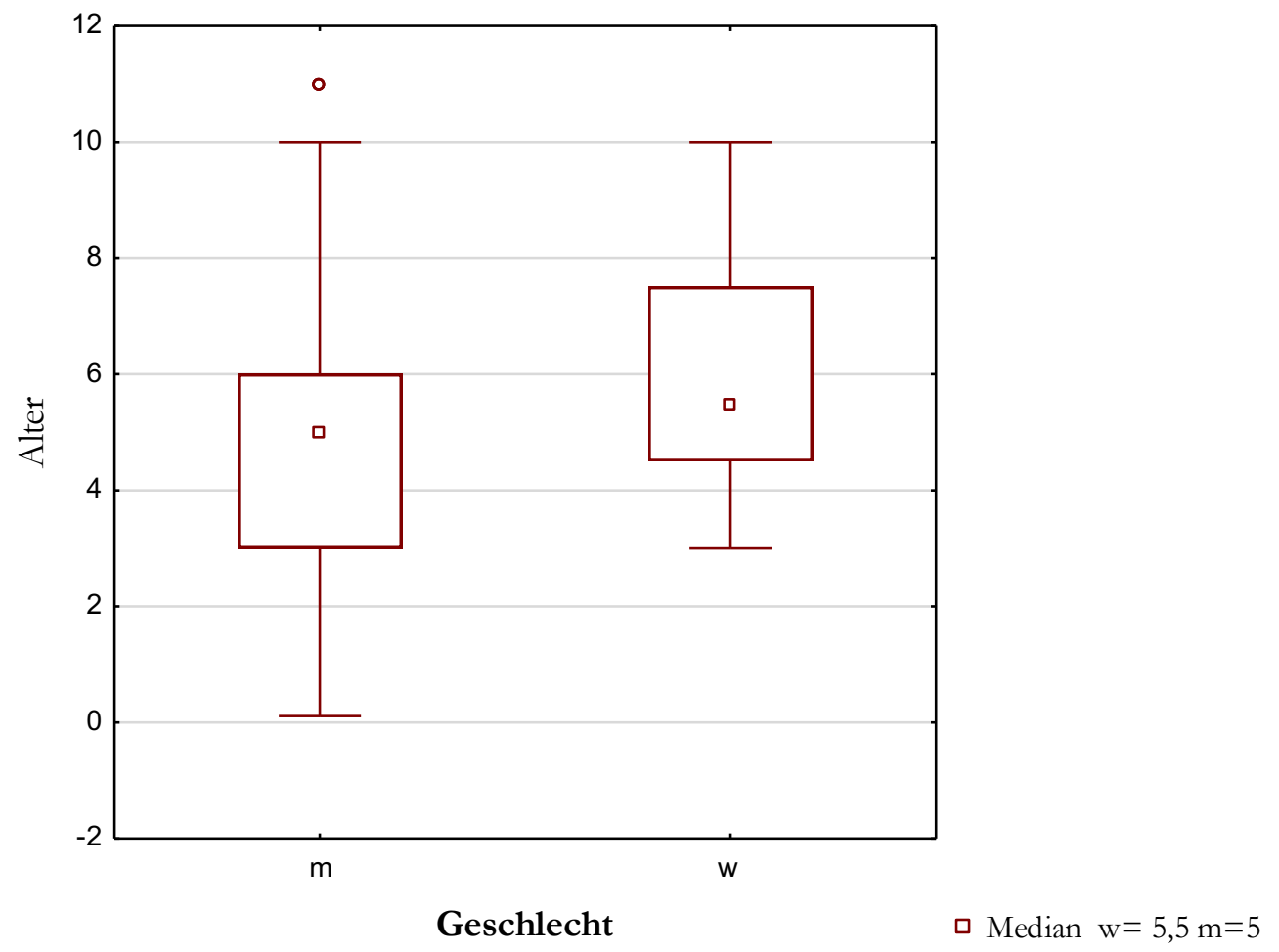

Abbildung 2: Altersverteilung abhängig vom Geschlecht. 
Zum Zeitpunkt der Operation variierte das Körpergewicht der Kinder zwischen minimal 8,5 $\mathrm{kg}$ und maximal $44 \mathrm{~kg}$. Der Median für das Gesamtgewicht liegt bei 18,5 kg (s. Abbildung 3).

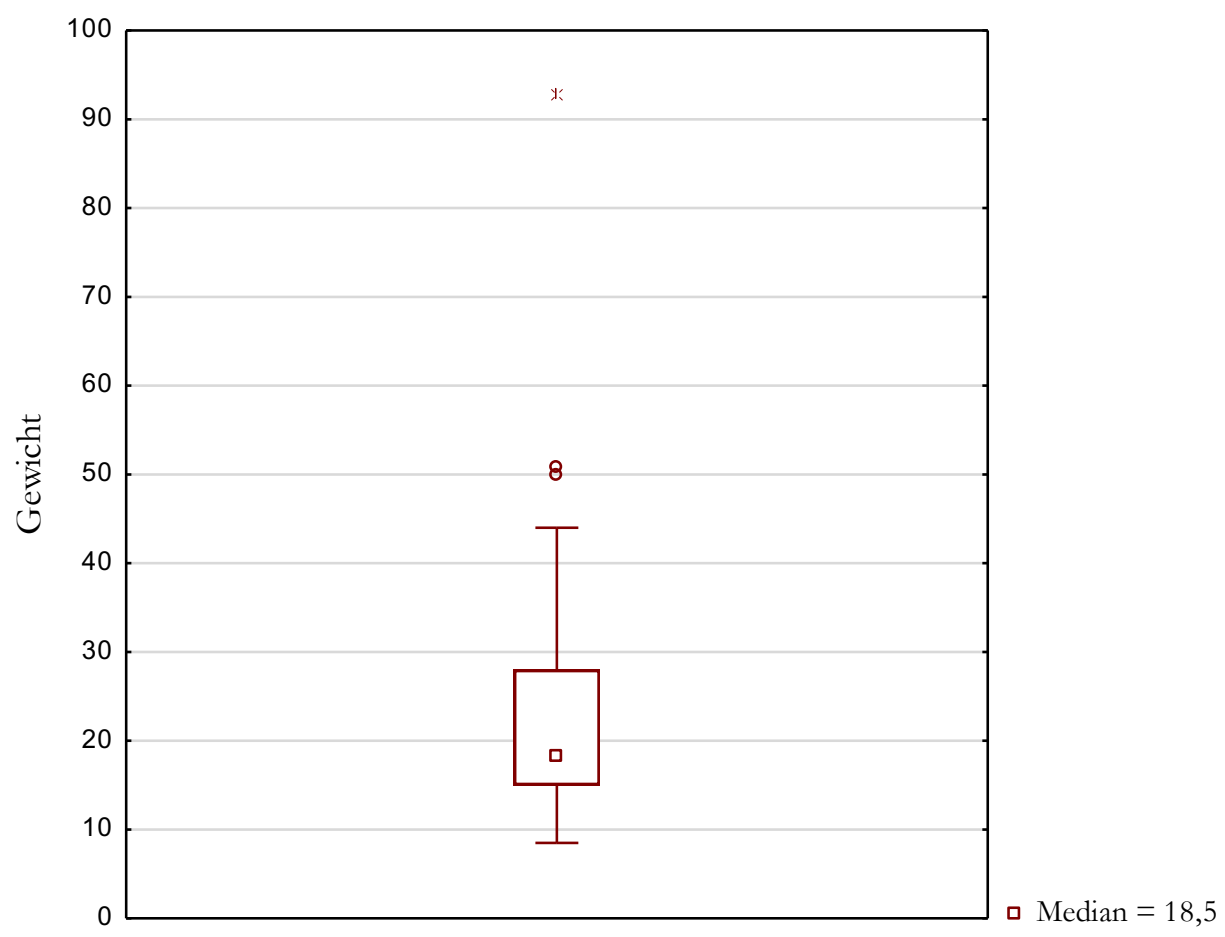

Abbildung 3: Gewichtsverteilung aller untersuchten Kinder.

\subsection{Operationstechnische Daten}

Die am Häufigsten beobachteten Diagnosen in der ambulanten urologischen Kinderchirurgie waren im gewählten Zeitraum die Phimose (fünfzig von hundert vorstelligen Patienten), der Gleit- und Leistenhoden (jeweils elf Patienten) und die Leistenhernie mit neun diagnostizierten Patienten. Weitere Konsultationsgründe waren unter anderem zwei Atherome, zwei Lipome, drei Patienten mit einer Hydrozele, eine Labiensynechie und ein Ankyloglosson, sieben Präputialverklebungen, zwei Warzenextirpationen (Verrucae vulgares) sowie eine Nabelhernie (s. Abbildung 4). Dementsprechend ist die Zirkumzision mit fünfzig beobachteten Fällen, die am regelmäßigsten durchgeführte Operation. Bei 22 Kindern wurde in den elf Monaten eine Orchidopexie durchgeführt, wobei sechs der Kinder eine beidseitige Orchidopexie erhielten. Bei einem Großteil der Patienten (elf Kinder) wurde der rechte Hoden fixiert, nur fünf Patienten wurden am linken Hoden operiert. Zehn Kinder mit Leistenhernie oder Nabelhernie unterzogen sich einer Herniotomie. In sieben Fällen war eine Präputiolyse zur Weitung der Vorhaut ausreichend. Die Lipome, die Verrucae vulgares und die zwei Atherome wurden exzidiert, die drei Hydrozelen resiziert. Nur einmal erfolgte ein operativer Eingriff zum Lösen der Labien, ebenfalls einmalig erfolgte die Frenotomie.

Neunzehn Kinder wurden bereits in der Vergangenheit operiert, für 81 Kinder war der vorgesehene Eingriff die erste Operation. 


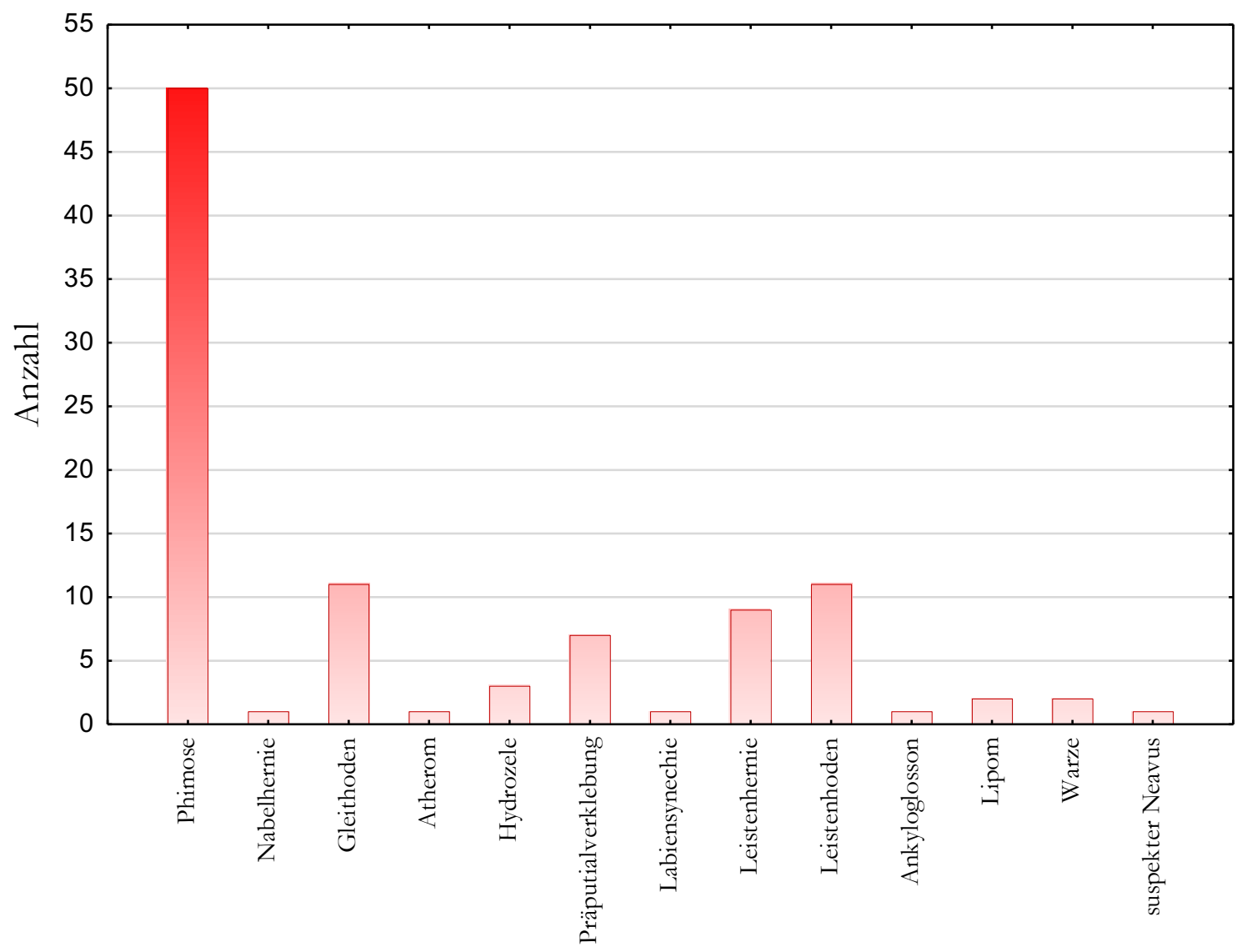

Abbildung 4: Diagnosen.

Bei allen Patienten erfolgte der chirurgische Eingriff in Allgemeinnarkose. Zusätzlich wurde bei 69 Patienten eine Regionalanästhesie in Form eines Peniswurzelblockes (48 Fälle bei $\mathrm{n}=100$ ) oder einer Kaudalanästhesie (21 Fälle bei n=100) durchgeführt (s. Abbildung 5,6 ).

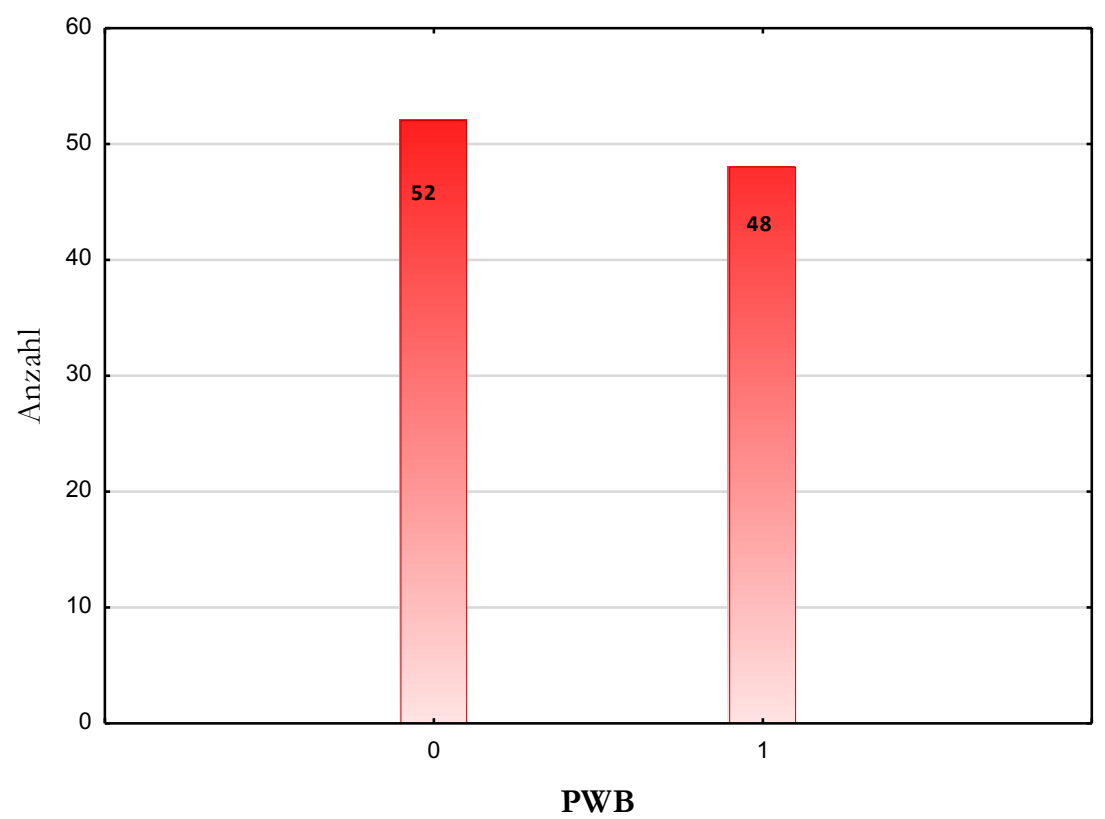

Abbildung 5: Anzahl der verwendeten Peniswurzelblöcke $(0=$ nein, $1=$ ja). 


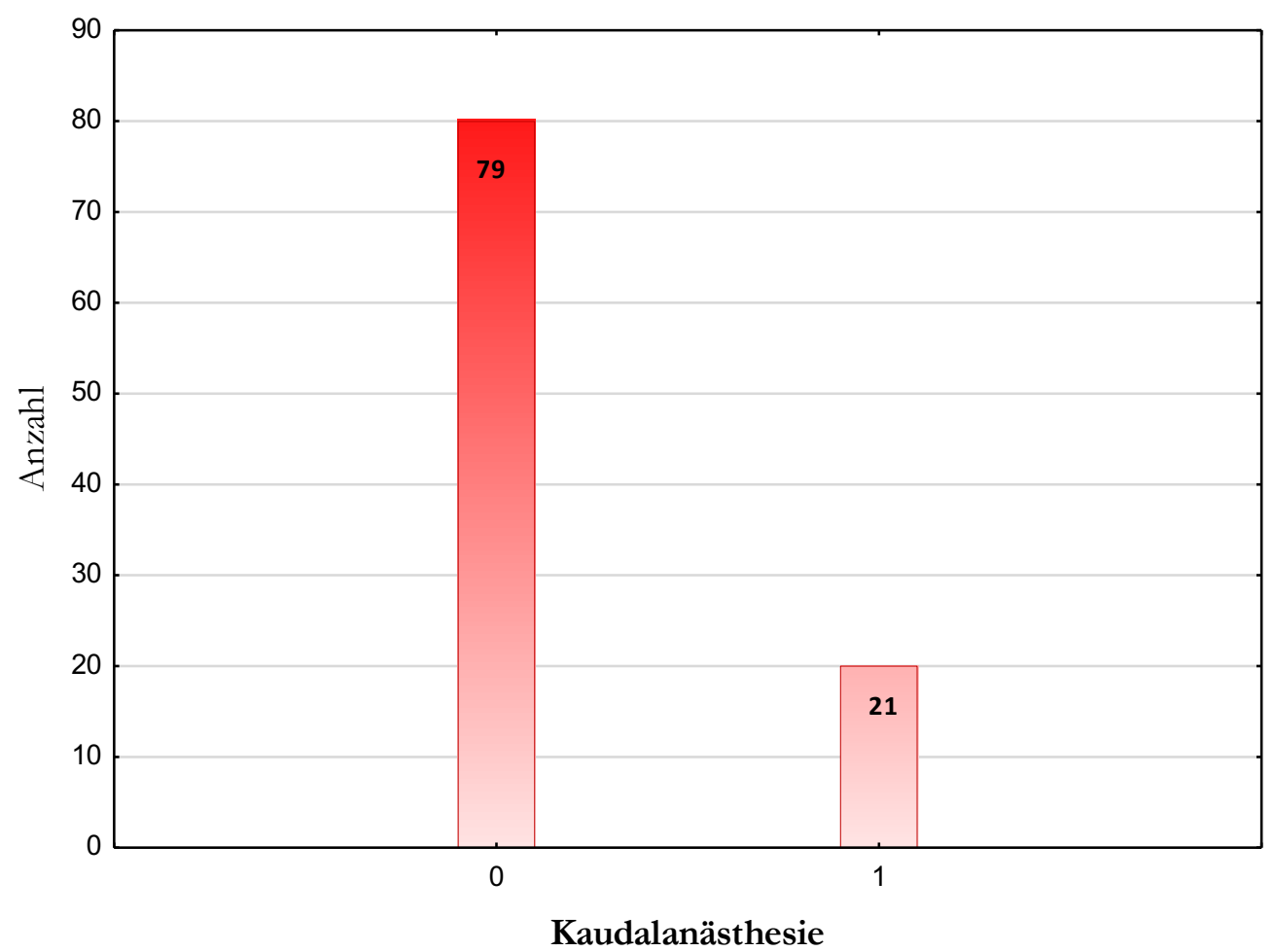

Abbildung 6: Anzahl der verwendeten Kaudalanästhesien ( $0=$ nein, $1=$ ja).

Während der Allgemeinnarkose erfolgte die Atemwegssicherung meist mit Hilfe einer Larynxmaske, nur bei fünf Kindern wurde ein Tubus verwendet (s. Abbildung 7).

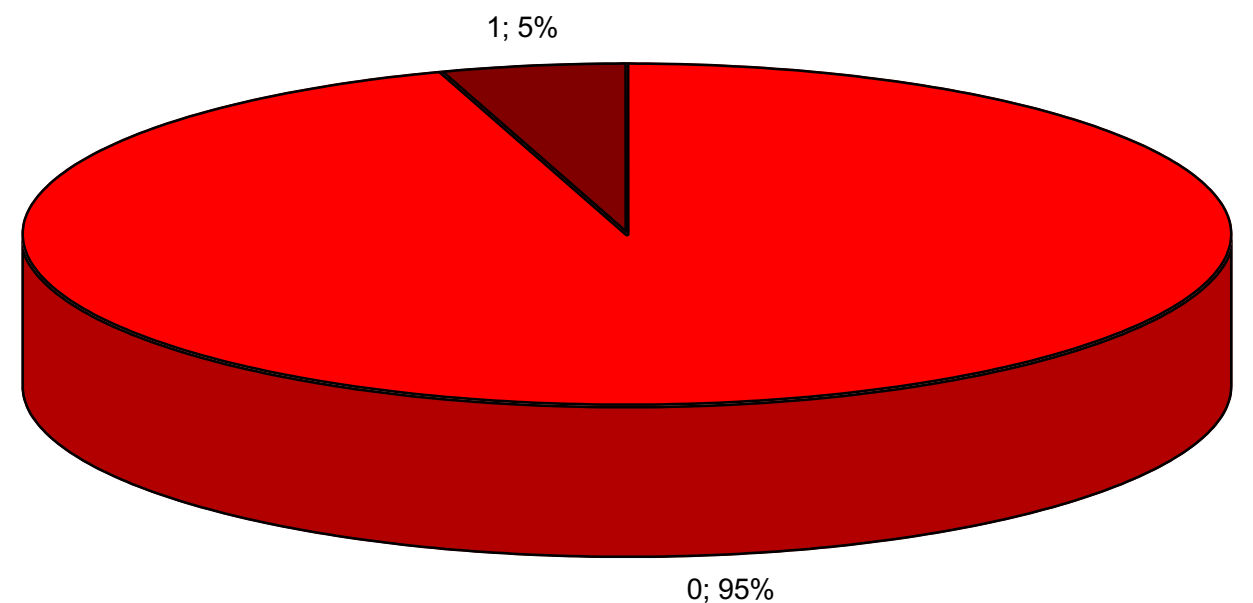

\section{Intubationsnarkose}

Abbildung 7: Intubation mit einem Tubus $(0=$ nein, $1=$ ja $)$. 
Die durchschnittliche Operationszeit (berechnet anhand der Narkosedauer) betrug bei allen Operationen jeweils 50, $29 \pm$ 2,12 Minuten. Der Median beträgt $49(\mathrm{n}=100)$ (s. Abbildung 8).

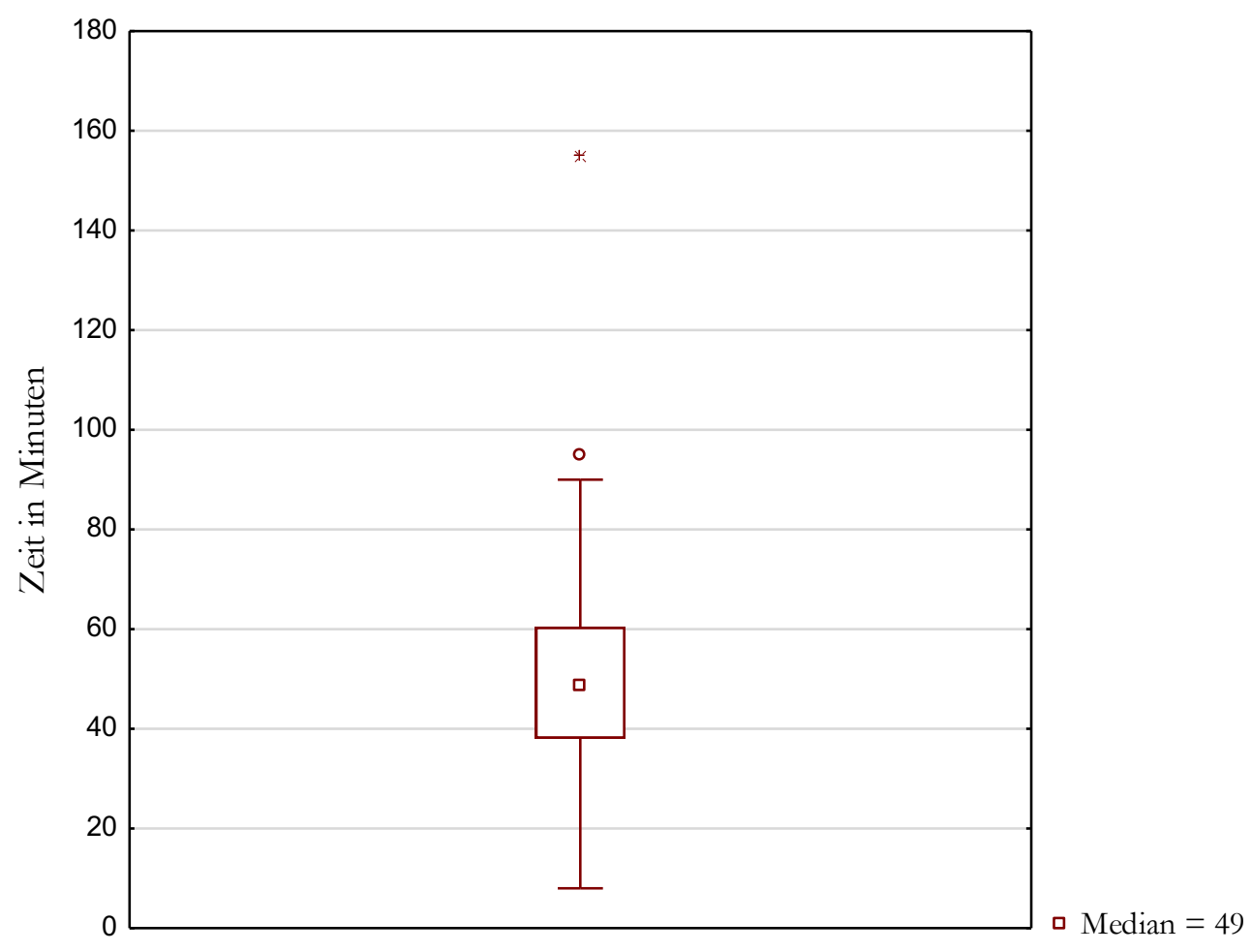

Abbildung 8: Operationszeit in Minuten.

Ein weiterer Aspekt der präoperativen Daten waren die Angaben bezüglich einer Voroperation. Für 81 der hundert Kinder war der durchgeführte Eingriff die erste Operation, 19 Kinder wurden bereits in der Vergangenheit operiert.

\subsection{Einfluss des neuen Behandlungskonzeptes}

Verschiedene Parameter, bestehend aus postoperativen Symptomen, Bestandteilen des perioperativen Managements und der Organisationsstruktur des Operationstages, wurden evaluiert und hinsichtlich ihres Einflusses durch das neue Behandlungskonzept untersucht. Hierzu wurden diverse Korrelationen durchgeführt und zur weiteren Diskriminierung ausgewählte Daten verglichen. Im Folgenden werden entsprechend der Themenkomplexe die für die Auswirkungen des Behandlungskonzeptes relevanten, deskriptiven Daten aufgeführt.

\subsubsection{Postoperative Symptome}

Am ersten postoperativen Tag wurden die Eltern telefonisch kontaktiert, um eventuell aufgetretene Symptome zu erfassen. Die erfragten Symptome beinhalteten eine Schwellung im Bereich des Operationsgebietes, Übelkeit und Erbrechen, mögliche Nachblutungen und andauernde Müdigkeit des Kindes. Von den insgesamt hundert befragten Eltern gaben 
vierzig an, dass ihre Kinder beschwerdefrei und ohne jegliche Komplikationen seien. Bei 41 Kindern trat jeweils eine der genannten Symptome auf. 14 Patienten beklagten zwei aufgetretene Symptome und fünf Kinder hatten nach der Operation zu Hause Probleme mit drei der vier evaluierten Faktoren (s. Abbildung 9). Postoperative Schmerzen wurden unabhängig der genannten Faktoren evaluiert und im Folgenden separat ausgewertet.

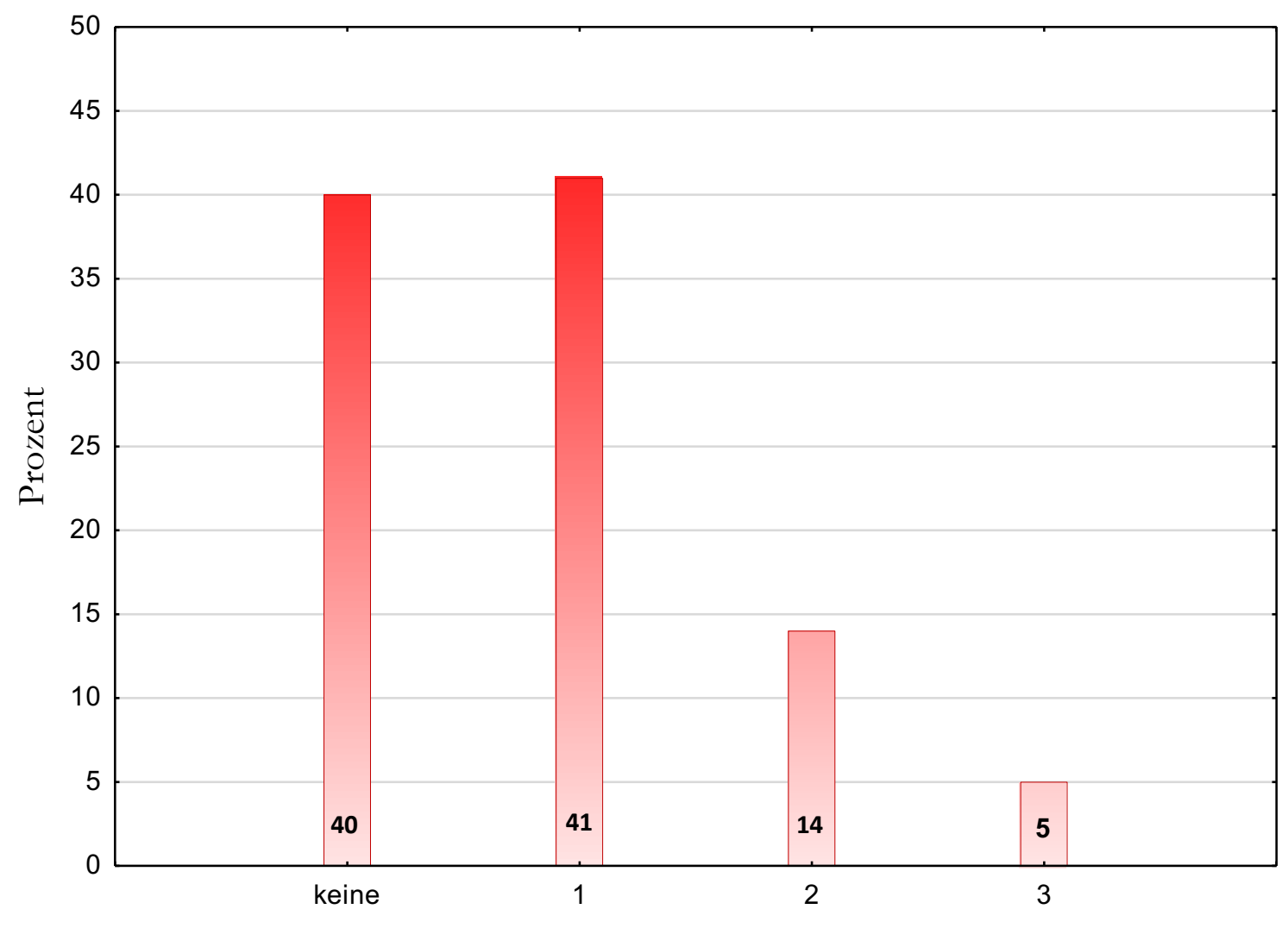

Anzahl postoperativer Symptome

Abbildung 9: Postoperative Symptome. 
Das prozentual am häufigsten vertretene postoperative Symptom war die Schwellung des Operationsgebietes mit 38 beschriebenen Fällen (im Folgenden jeweils $n=100$ ). Die absolute Häufigkeit der Kinder, die am ersten postoperativen Tag noch deutliche Müdigkeit zeigten, betrug 24. Elf Kinder litten weiterhin unter starker Übelkeit, insgesamt sechs der hundert Kinder erbrachen sich postoperativ (s. Abbildung 10).

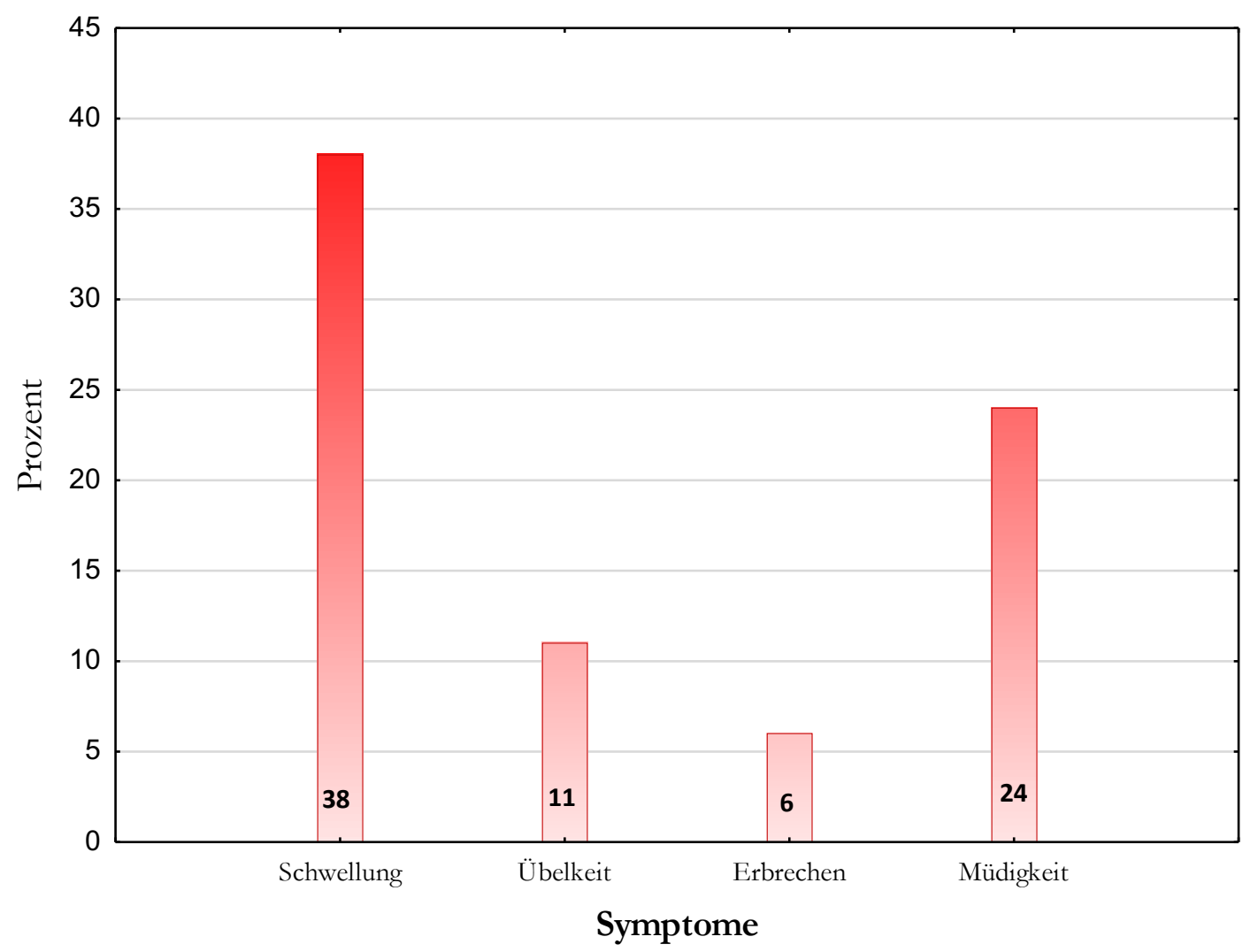

Abbildung 10: Absolute Häufigkeit aufgetretener Symptome. 
Die Schmerzen der Kinder im häuslichen Umfeld wurden ebenfalls telefonisch ermittelt. Hierzu ordneten die Eltern die vermuteten Schmerzen ihrer Kinder auf einer Schmerzskala von null $=$ kein Schmerz bis hin zu fünf= unerträglicher Schmerz ein. 29 Elternteile vermuteten bei ihren Kindern Schmerzfreiheit und gaben den Zahlenwert null an. Sechs Eltern wählten im Gespräch die eins, 24 die zwei, 21 die drei und 18 Eltern die vier. Unerträgliche Schmerzen (Skalenwert 5) wurden von fünf Eltern angegeben (s. Abbildung 11).

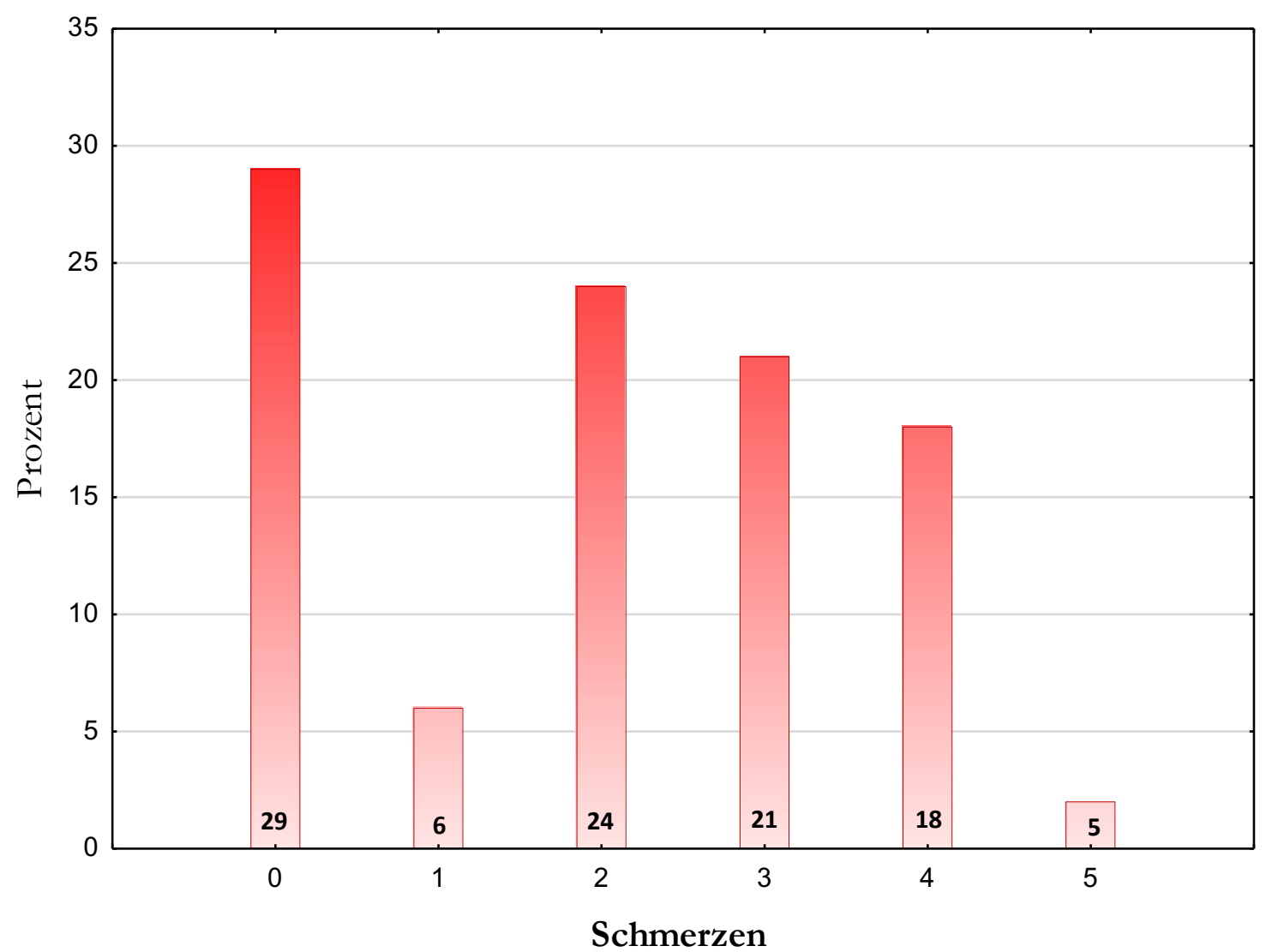

Abbildung 11: Schmerzangaben anhand der Schmerzskala $(0=$ kein Schmerz bis $5=$ unerträglicher Schmerz). 
Zur Verlaufskontrolle wurden die Eltern im Rahmen der Studie am fünften Tag postoperativ noch einmal kontaktiert. Hier wurde unter anderem auf die Wundheilung Bezug genommen. Die Eltern bewerteten jeweils die Aussage: „Die Wundheilung verlief soweit problemlos“ mit den Antwortmöglichkeiten 1=trifft gar nicht zu bis 5= trifft völlig zu. $92 \%$ der Eltern schätzten die Wundheilung als problemlos ein und wählten die 5. Kategorie (s. Tabelle 3).

Tabelle 3: Postoperativer Verlauf der Wundheilung.

\begin{tabular}{|l|r|c|r|r|}
\hline \multirow{2}{*}{ Kategorie } & \multicolumn{4}{|l|}{ Häufigkeitstabelle: Wundheilung } \\
\cline { 2 - 5 } & Häufigkeit & $\begin{array}{c}\text { Kumulative } \\
\text { Häufigkeit }\end{array}$ & \multicolumn{1}{c|}{ Prozent } & $\begin{array}{c}\text { Kumulativ } \\
\text { Prozent }\end{array}$ \\
\hline 1 & 2 & 2 & 2,00000 & 2,0000 \\
\hline 2 & 2 & 4 & 2,00000 & 4,0000 \\
\hline 3 & 1 & 5 & 1,00000 & 5,0000 \\
\hline 4 & 3 & 8 & 3,00000 & 8,0000 \\
\hline 5 & 92 & 100 & 92,00000 & 100,0000 \\
\hline Missing & 0 & 100 & 0,00000 & 100,0000 \\
\hline
\end{tabular}

\subsubsection{Organisationsstruktur}

Unterschiedliche Aspekte des Fragebogens zielten darauf ab, den Ablauf des Operationstages genauer zu beurteilen. Hierzu gehörte unter anderem die Frage nach den Kontaktdaten eines medizinischen Ansprechpartners im Falle von Schwierigkeiten.

1. Item zur Bewertung der Kontaktdaten:

"Ich wusste an welche Person ich mich im Falle von Fragen und Problemen wenden konnte."

Diese Aussage wurde von allen Eltern mit „trifft völlig zu“ beantwortet.

Weiterhin wurde konkret die Struktur des Operationstages angesprochen. Die Frage nach eben dieser Struktur bezog sich auf die präoperativ vermittelten Informationen bezüglich des Ablaufes und der Organisation des Operationstages und sollte mit Hilfe folgender Aussage bewertet werden.

1. Item zur Bewertung der Struktur des Operationstages:

„Der Tag der Operation war für mich klar strukturiert und vorbersebbar." 
Die große Mehrheit der Eltern antwortete mit 5=trifft völlig zu. Vier Elternteile bewerteten die Aussage mit $1=$ trifft gar nicht zu. Die verbliebenen fünf Elternteile entschieden sich für die Antwortmöglichkeiten zwei, drei oder vier (s. Abbildung 12).

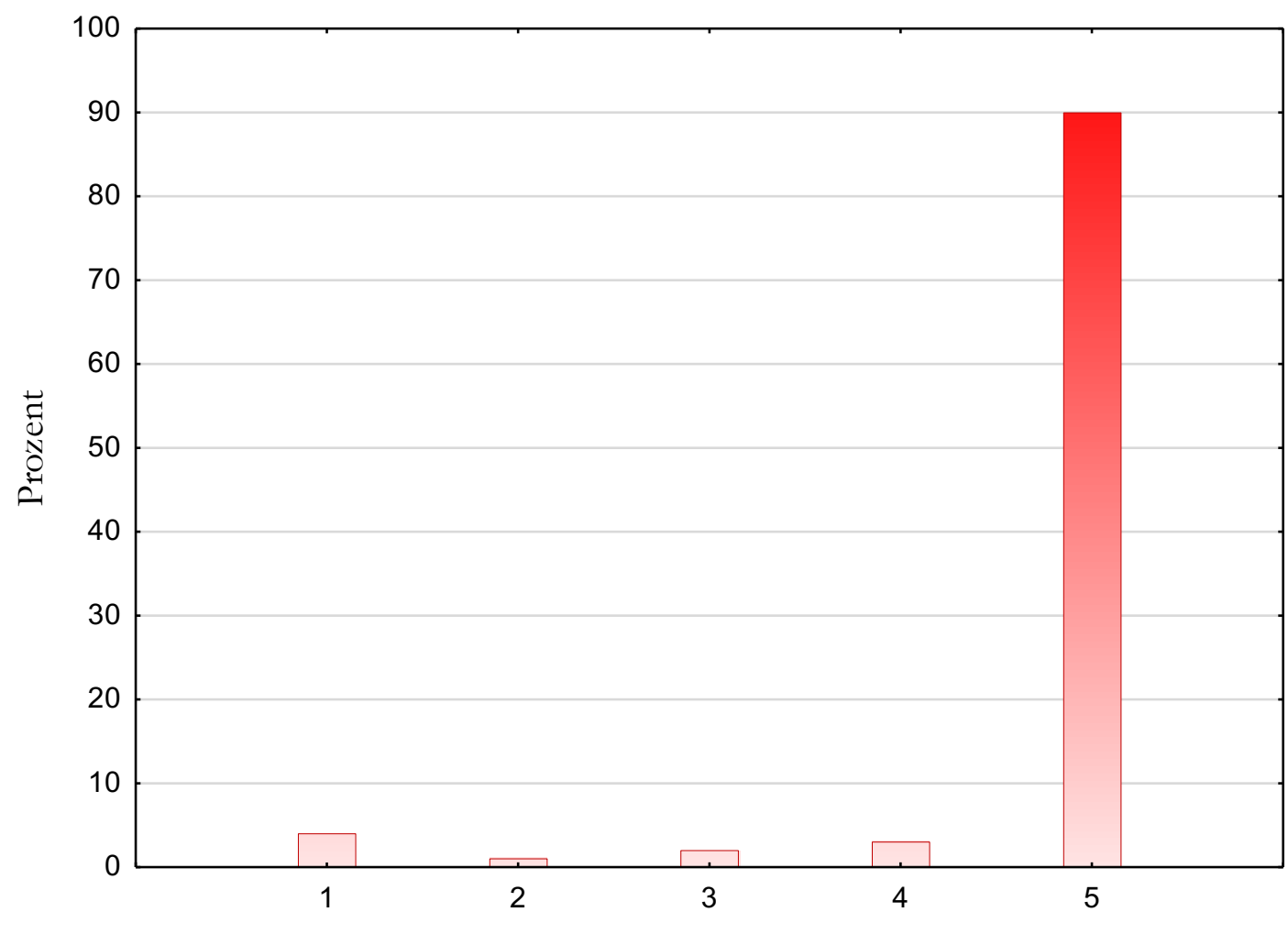

Zufriedenheit mit der Struktur des Operationstages

Abbildung 12: Zufriedenheit mit der Struktur des Operationstages $(0=$ keine Angabe, $1=$ trifft gar nicht $\mathrm{zu}, 2=$ trifft eher nicht $\mathrm{zu}, 3=$ unentschieden, $4=$ trifft eher $\mathrm{zu}, 5=$ trifft völlig zu).

Neben der Struktur des Operationstages wurde auch der Ablauf des Operationstages bewertet. Hier sollte rückblickend die postoperative Sicht der Eltern auf den tatsächlichen Ablauf des Tages beleuchtet werden. Insgesamt 93 Eltern gaben an, mit dem Ablauf des Operationstages zufrieden zu sein.

Folgendes Item wurde zur Evaluation verwendet:

1. Item zur Bewertung des Ablaufes am Operationstag:

„Waren Sie mit dem Ablauf im Krankenhaus am Tag der Operation zufrieden?"

Die Wartezeiten, ebenfalls Parameter eines adäquaten Zeitmanagements, wurden in die präund postoperative Wartezeit differenziert. Der präoperative Zeitraum bezog sich auf das Intervall von der Anmeldung in der Ambulanz des Zentral-OPs bis zum Beginn der 
Narkoseeinleitung. Die Zeitspanne von der Ankunft im Aufwachraum bis zur Entlassung wurde durch die postoperative Wartezeit repräsentiert.

Die durchschnittliche präoperative Wartezeit von 76 Kindern betrug 60,6 \pm 6 min, bei den verbliebenen 24 Kindern konnten die Eltern postoperativ keine genauen Angaben machen. Die Daten fehlen an dieser Stelle.

Postoperativ blieben die Kinder im Mittel 173,4 \pm 8,4 min im Aufwachraum bis zur Entlassung nach Hause.

Einige Patienten bekamen präoperativ ein kurz wirksames Benzodiazipin zur Anxiolyse. In dreizehn Fällen wurde vor der Operation gewichtsadaptiert Midazolam verabreicht.

Präoperativ wurde allen Eltern aus Sicherheitsgründen die Anwesenheit einer zweiten erwachsenen Begleitperson für den Transport nach Hause empfohlen. Ein Elternteil sollte sich während der Autofahrt ausschließlich auf das Kind konzentrieren können. 95\% aller Eltern hielten sich an diese Empfehlung, nur 5\% holten ihr Kind ohne eine zweite volljährige Begleitung nach der Operation ab.

\subsubsection{Perioperatives Management}

Das perioperative Management beinhaltet multiple Einflussfaktoren. Im Folgenden wird auf die Aufklärung durch das medizinische Personal, das Schmerzmanagement durch die Eltern sowie auf die Nachsorge durch den Kinderarzt eingegangen.

Alle Eltern bewerteten die postoperative Arztkonsultation. 95\% der Eltern gaben an, dass das Gespräch mit den Kinderchirurgen nach der Operation alle Fragen beantwortet habe. Lediglich 5 Elternteile bewerteten dieses Gespräch als unvollständig.

Rückwirkend wurde außerdem am fünften postoperativen Tag die Qualität der Aufklärung im Allgemeinen evaluiert. Hierzu wurde folgende Aussage bewertet:

1. Item zur Bewertung der Aufklärungsqualität:

„In Vorbereitung auf die Operation bätte ich mir eine detailliertere Aufklärung gewünscht."

Die Beurteilung erfolgte auch hier anhand von fünf Antwortmöglichkeiten (1 =trifft gar nicht $\mathrm{zu}$ bis $5=$ trifft völlig $\mathrm{zu})$. Ein Elternteil beantwortete diese Frage nicht $(0=$ nicht beantwortet), die Mehrheit der Eltern (58 Fälle von $n=99$ ) wünschten sich keine detailliertere Aufklärung (s. Abbildung 13). 


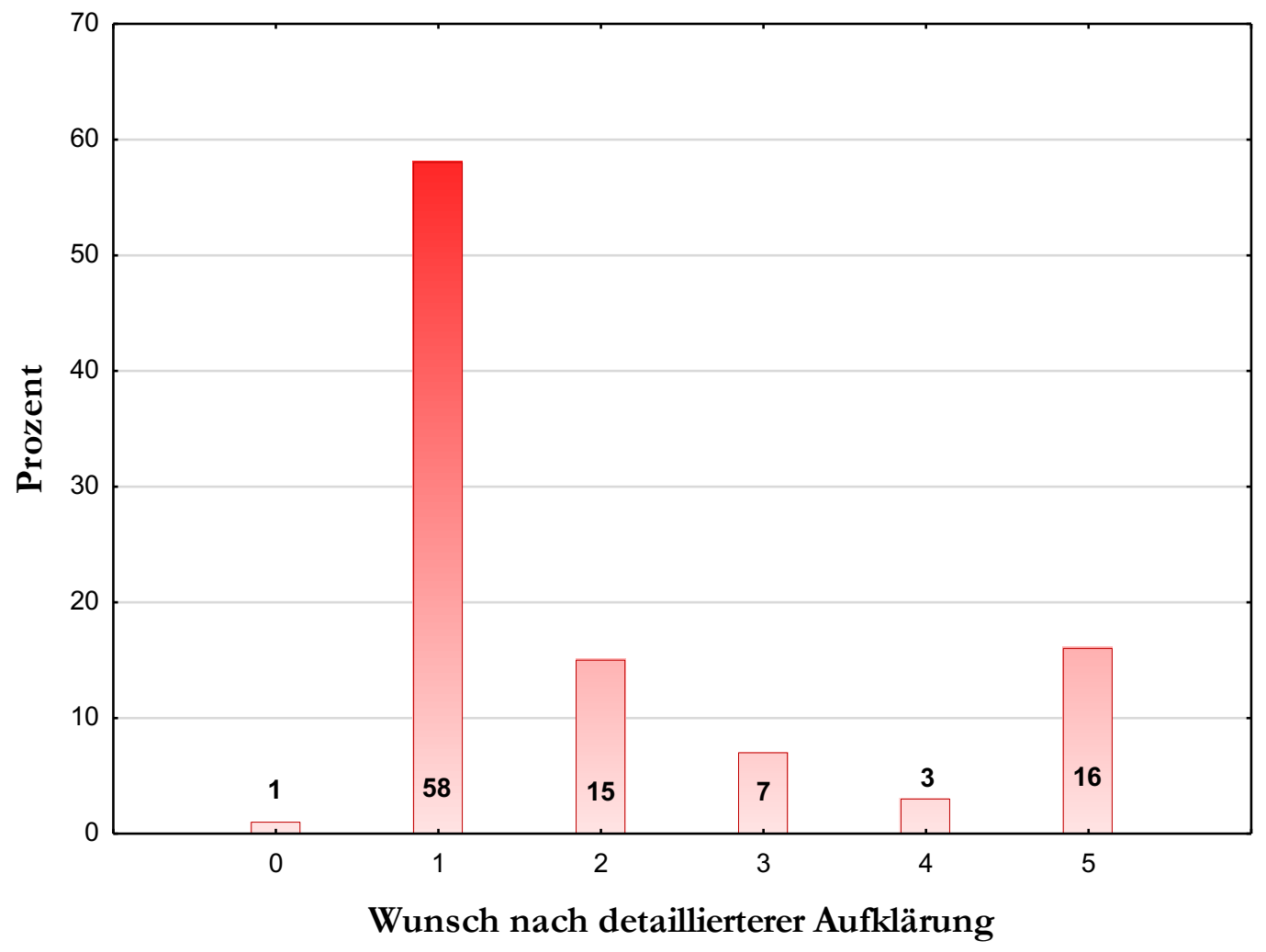

Abbildung 13: Zusammenfassende Bewertung der präoperativen Aufklärung ( $0=$ keine Angabe, $1=$ trifft gar nicht $\mathrm{zu}, 2=$ trifft eher nicht $\mathrm{zu}, 3=$ unentschieden, $4=$ trifft eher $\mathrm{zu}$, $5=$ trifft völlig $\mathrm{zu})$. 
Die Eltern der Kinder wurden präoperativ über möglicherweise auftretende Schmerzen, Blutungen und verschiedene Narkoseverfahren informiert. Die jeweiligen Aufklärungen wurden während der Befragung differenziert und anhand von drei Aussagen separat ausgewertet.

1. Item zur Evaluation der Schmerzaufklärung:

„Ich bin ausreichend über eine mögliche Schmerssituation ₹u Hause aufgeklärt worden."

Von hundert befragten Eltern gab nur eine Person an, nicht ausreichend informiert worden $\mathrm{zu}$ sein (Skalenwert $1=$ trifft überhaupt nicht $\mathrm{zu}$ ). In allen übrigen Fällen wählten die Befragten die 5= trifft völlig zu. Die Antwortmöglichkeiten zwei bis vier wurden nicht angegeben (s. Abbildung 14). Außerdem gaben 99\% der Eltern an, postoperativ mit den Schmerzen des Kindes adäquat umgehen zu können.

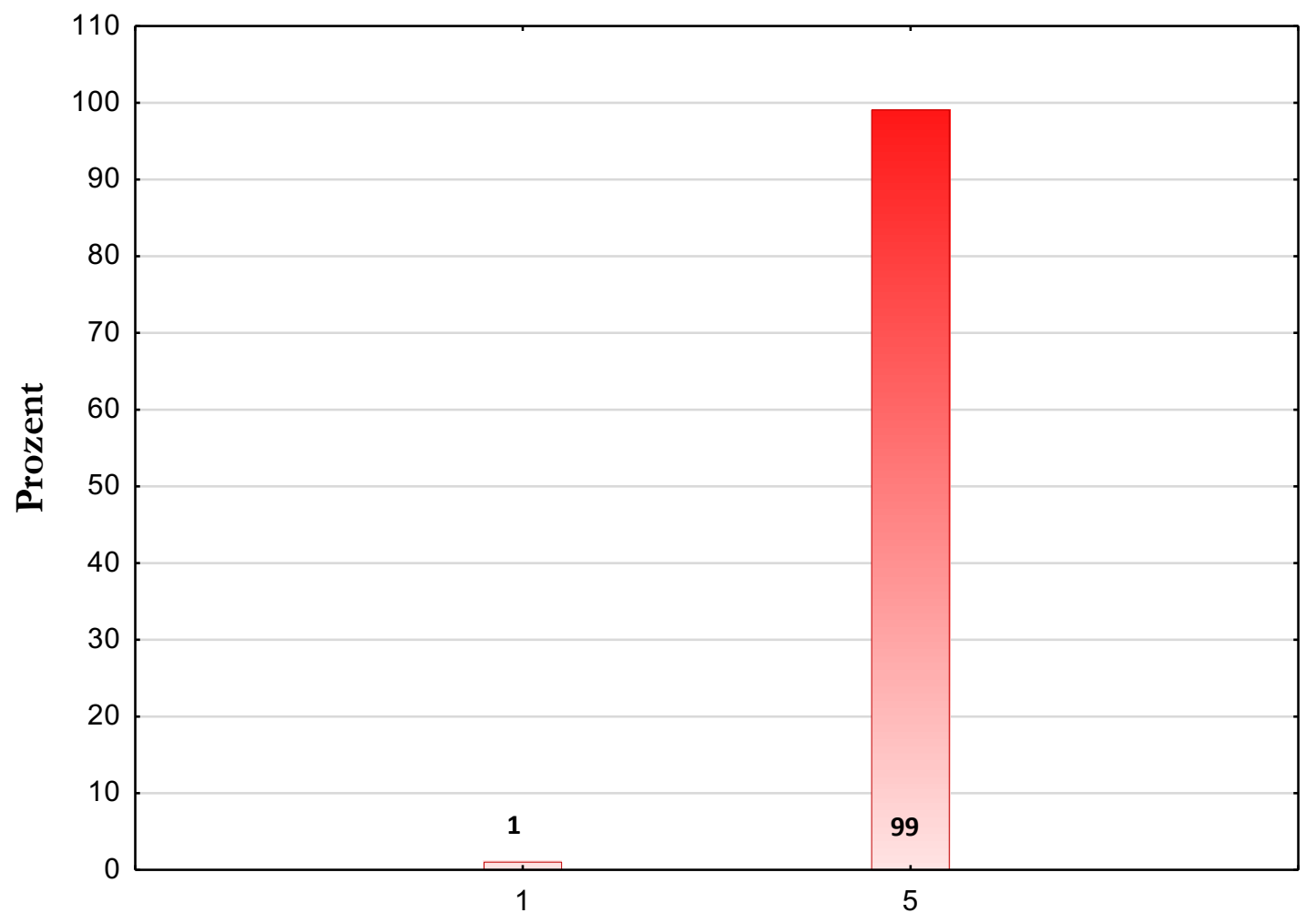

Aufklärung bezüglich möglicher Schmerzen

Abbildung 14: Zufriedenheit mit der Aufklärung bezüglich möglicher Schmerzen $(0=$ keine Angabe, $1=$ trifft gar nicht zu, 2= trifft eher nicht zu, 3= unentschieden, $4=$ trifft eher zu, $5=$ trifft völlig zu). 
2. Item zur Evaluation der Aufklärung bezüglich Nachblutungen:

„Ich bin vor der Operation ausreichend über eventuell auftretende Nachblutungen informiert worden."

61\% der Befragten ( $\mathrm{n}=100)$ stimmten einer ausreichenden Aufklärung über auftretende Nachblutungen völlig zu. 11\% der Erziehungsberechtigten fühlten sich unzureichend aufgeklärt. Keine Aussage machten 5\% aller kontaktierten Eltern. Die verbliebenen 28\% ordneten ihre Antworten zwischen den Möglichkeiten zwei und vier ein (s. Abbildung 15).

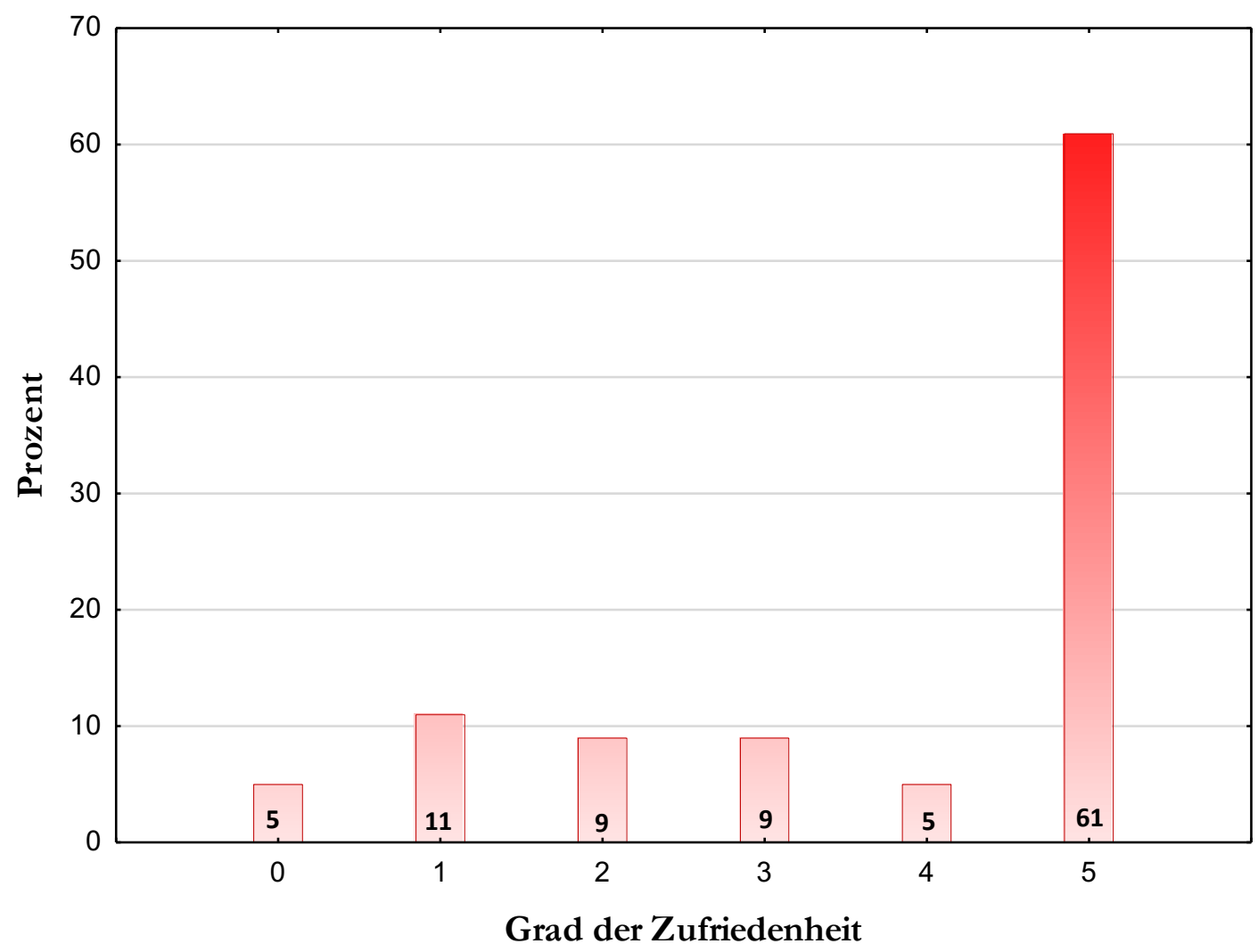

Abbildung 15: Zufriedenheit mit der Aufklärung bezüglich möglicher Nachblutungen $(0=$ keine Angabe, $1=$ trifft gar nicht zu, $2=$ trifft eher nicht zu, $3=$ unentschieden, $4=$ trifft eher zu, $5=$ trifft völlig zu). 
3. Item zur Evaluation der Aufklärung über Narkoseverfahren:

„Ich wurde ausreichend über das gewäblte Narkoseverfahren aufgeklärt."

Hier gab es als Antwortmöglichkeiten ,ja“" oder ,nein“. Insgesamt $75 \mathrm{Mal}(\mathrm{n}=100)$ stimmten die Eltern einer ausreichenden Aufklärung zu. In einem Viertel aller Fälle hatten die Eltern nicht den Eindruck hinreichend informiert worden zu sein (s. Abbildung 16).

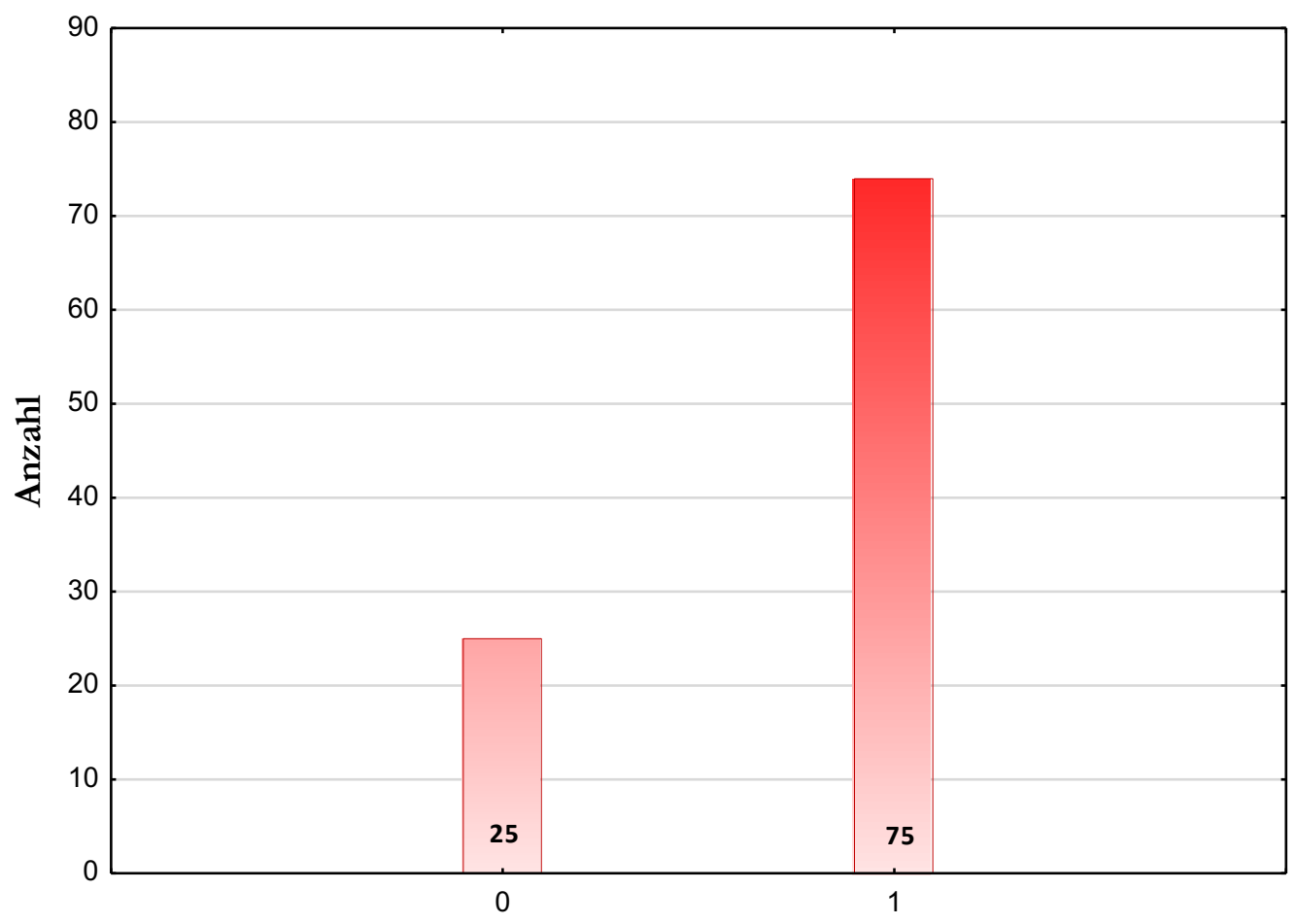

Aufklärungszufriedenheit Narkoseverfahren

Abbildung 16: Zufriedenheit mit der Aufklärung über das Narkoseverfahren $(0=$ nein, $1=$ ja $)$.

Ein Item des Fragebogens zielte noch einmal explizit auf die Dauer der Nahrungskarenz an. Hier sollten die Eltern angeben, ob Ihnen der Zeitpunkt, an dem das Kind das letzte Mal etwas Essen und etwas Trinken darf, bewusst ist und ihn auch auf dem Fragebogen reproduzieren.

1. Item zur Evaluation der Nahrungskarenz:

„Mit ist bekannt, wann mein Kind das letrte Mal etwas Essen und Trinken darf."

Insgesamt 84 Eltern gaben an, den Zeitpunkt zu wissen (entspricht „trifft völlig zu“ bei der Itembewertung) und trugen ihn auch entsprechend korrekt in die Bögen ein. Nur fünf Elternteile bewerteten die Aussage mit trifft eher zu, zwei wählten „unentschieden“ und neun gaben keine Antwort. 
Die geplante Nachsorgeuntersuchung durch den Kinderarzt fand bei 93\% der befragten Eltern volle Unterstützung, nur 7\% sprachen sich gegen eine ambulante Nachsorge und für einen Untersuchungstermin im Klinikum aus (s. Abbildung 17).

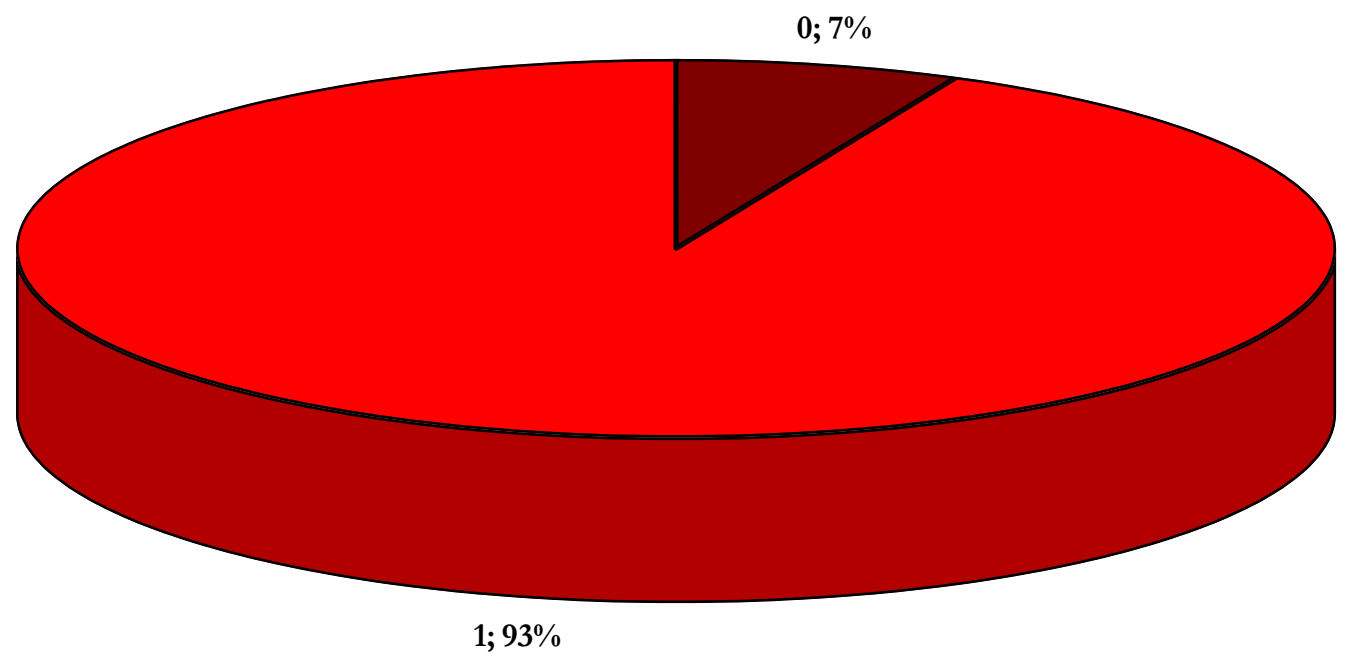

Nachsorgeuntersuchung durch den Kinderarzt

Abbildung 17: Zustimmung mit einer Nachsorgeuntersuchung durch den Kinderarzt $(0=$ nicht gewünscht, $1=$ gewünscht $)$. 


\subsection{Korrelationsanalyse und Einfluss des neuen Behandlungskonzeptes}

Im Folgenden werden einzelne Faktoren hinsichtlich der Auswirkung auf die Zufriedenheit mit dem perioperativen Management untersucht. Weiterhin wird der Einfluss einzelner Aspekte des neuen Behandlungskonzeptes auf das perioperative Management näher beleuchtet.

\subsubsection{Operationstechnische Daten}

Die Eltern wurden in dem präoperativen Fragebogen gebeten anzugeben, ob ihre Kinder in der Vergangenheit bereits operiert wurden. Diese Angaben wurden mit der allgemeinen Zufriedenheit der Eltern mit der Versorgung des Kindes in Korrelation gesetzt.

Hier zeigte sich, dass die allgemeine Zufriedenheit der Eltern signifikant mit der Angabe bezüglich der Voroperation korrelierte $(\mathrm{p}=0,0089)$. Die Eltern, deren Kinder zum ersten Mal operiert wurden, waren deutlich zufriedener mit der Versorgung des Kindes (s. Abbildung 18).

Die perioperativ verwendete Regionalanästhesie variierte je nach ursächlichem Krankheitsbild und durchgeführter Operation.

Unabhängig von der Art der Regionalanästhesie konnte kein signifikanter Zusammenhang zu postoperativ aufgetretenen Schmerzen nachgewiesen werden. Die Angaben der Angehörigen bezüglich der Schmerzen ihrer Kinder korrelierten weder mit der Anlage einer Kaudalanästhesie $(p=0,504928)$, noch mit der Verwendung eines Peniswurzelblockes $(p=0,504611)$.

\subsubsection{Postoperative Symptome}

Es stellte sich heraus, dass die beschriebenen Symptome unabhängig vom Alter des Kindes auftraten. Die Schwellung des Operationsgebietes $(p=0,997167)$, Übelkeit $(p=0.566791)$ und Erbrechen $(p=0,157033)$ sowie eine starke postoperative Müdigkeit $(p=0,437512)$ zeigten keine signifikante Korrelation mit den unterschiedlichen Altersgruppen der Kinder.

Allen Eltern wurde nach der Operation ihres Kindes mündlich empfohlen, nach wenigen Stunden postoperativ ein Schmerzmittel zu verabreichen. 50\% der Eltern bekamen diese Empfehlung zusätzlich in schriftlicher Form.

Nach Separation der beiden Fallgruppen zeigte sich, dass 41 von fünfzig Eltern, die lediglich mündlich informiert wurden, die verordnete Schmerzmittelgabe durchführten. Nach Einführung des Behandlungskonzeptes konnte eine Steigerung der Schmerzmittelgabe von $7 \%$ ermittelt werden. Jetzt gaben von den zusätzlich schriftlich informierten Eltern bereits 48 von fünfzig ihren Kindern das Schmerzmittel. 
Um den Einfluss der direkten postoperativen Schmerzen auf die Zeit bis zur Beschwerdefreiheit zu belegen, wurden die Daten zur postoperativen Schmerzangabe mit der Zeit bis zur absoluten Beschwerdefreiheit (in $\mathrm{h}$ ) in Korrelation gesetzt. Die durchschnittliche Zeit bis zur Beschwerdefreiheit der Kinder Betrug 9,5 \pm 1,62 Stunden (Median= 5). Kinder, die nach der Operation stärkere Schmerzen beklagten, benötigten deutlich mehr Stunden bis zur Schmerzfreiheit (s. Abbildung 18). Die angegebenen Skalenwerte in der Legende entsprechen jeweils den Zuordnungen auf einer Schmerzskala von $0=$ kein Schmerz bis $5=$ unerträglicher Schmerz).

Die Schmerzstärke des Kindes korreliert also hoch signifikant mit der Zeitspanne bis zur Beschwerdefreiheit $(\mathrm{p}=0,000027)$.

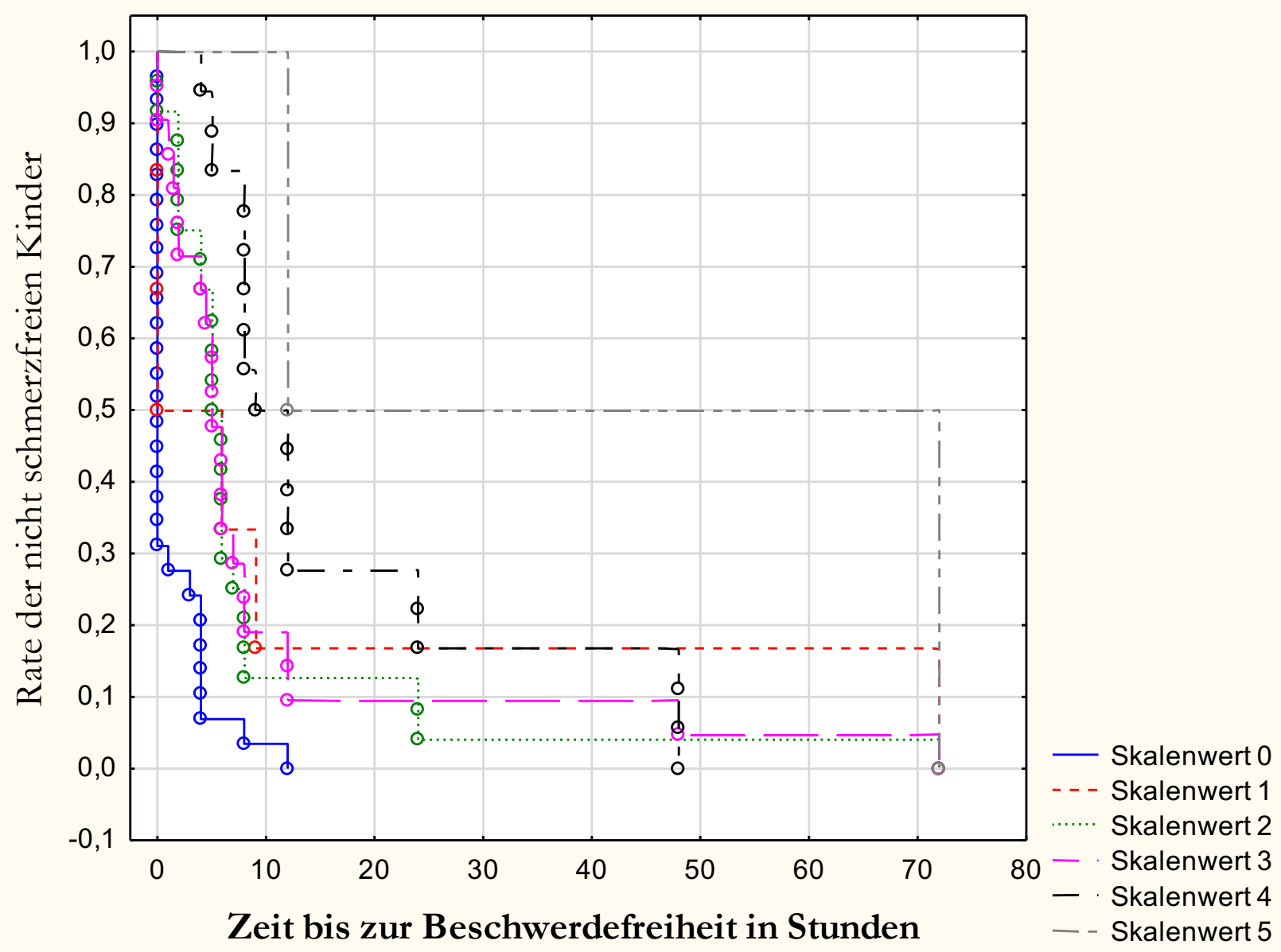

Abbildung 18: Subjektive Schmerzintensität in Bezug auf die Zeit bis zur Beschwerdefreiheit in Stunden dargestellt als Kaplan-Meier-Diagramm. 


\subsubsection{Organisationsstruktur}

Neben der Struktur des Operationstages wurde auch der Ablauf des Operationstages bewertet. Hier sollte rückblickend die postoperative Sicht der Eltern auf den tatsächlichen Ablauf des Tages beleuchtet werden. Insgesamt 93 Eltern gaben an, mit dem Ablauf des Operationstages zufrieden zu sein. Dies korrelierte hoch signifikant mit der allgemeinen Elternzufriedenheit mit der Versorgung des Kindes im Krankenhaus ( $p=0,00054)$.

Auch die bereits beschriebene Frage nach der Bewertung des Ablaufes am Operationstag wurde jeweils vor- und nach Einführung des erwähnten Behandlungsplanes beantwortet. Von den vor der Einführung befragten Eltern beantworteten bereits die deutliche Mehrheit (44 von $n=50$ ) die Frage mit ja und waren mit dem Ablauf des Operationstages vollzufrieden. Nur sechs Elternteile wiederum widersprachen und bewerteten das Item mit nein (s. Abbildung 19).

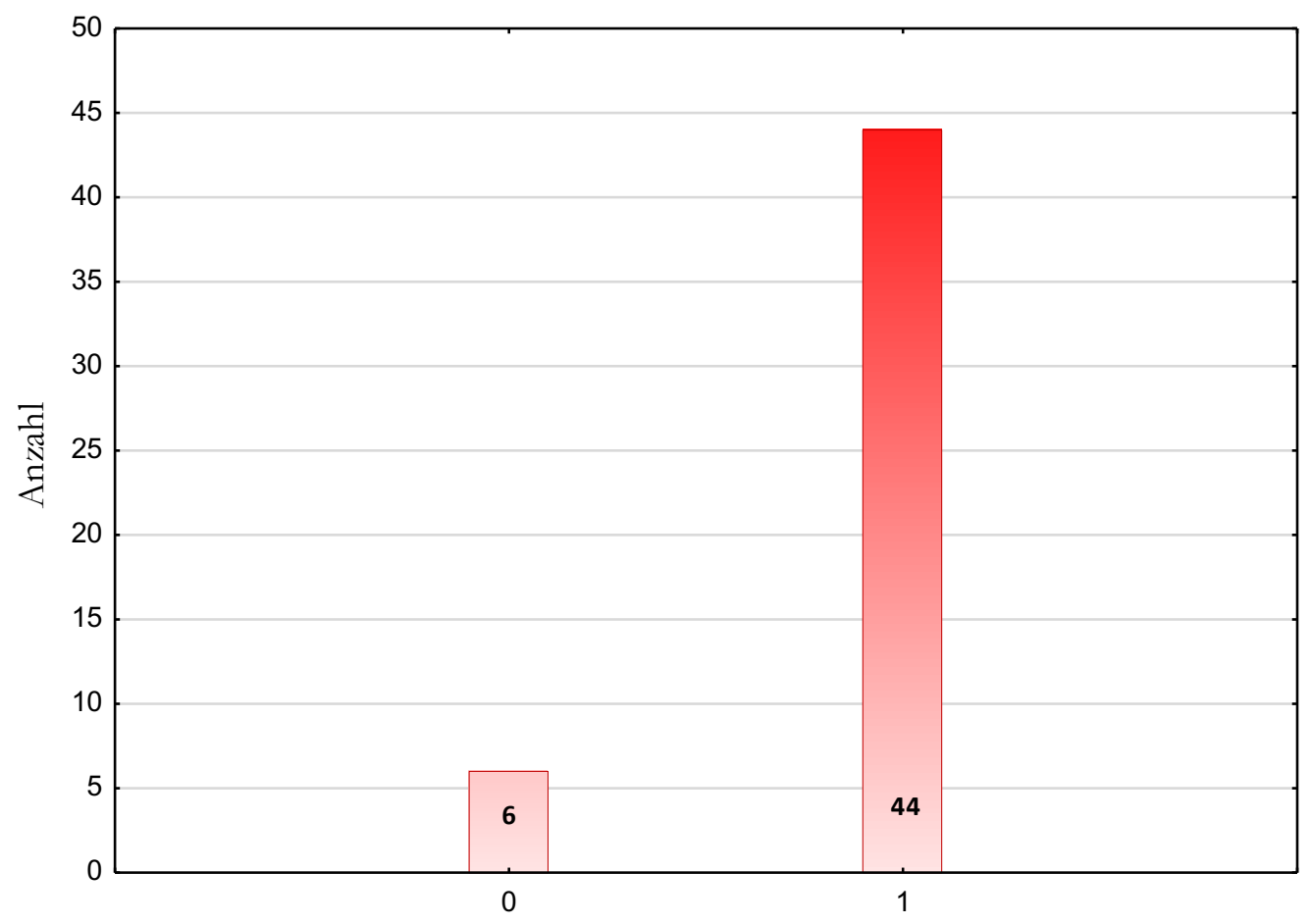

Ablaufzufriedenheit Fall 1-50

Abbildung 19: Zufriedenheit mit dem Ablauf des Operationstages vor Einführung des neuen Behandlungsplans $(0=$ nein, $1=\mathrm{ja})$. 
Nach der Einführung des Konzeptes wurde dieselbe Aussage („Waren Sie mit dem Ablauf im Krankenhaus am Tag der Operation zufrieden? ${ }^{*}$ ) nun von 99\% (49 von $n=50$ ) der befragten Elternteile mit ja beantwortet und von nur einer Person mit nein, was einer Steigerung der Ablaufzufriedenheit von 10\% entspricht (s. Abbildung 20).

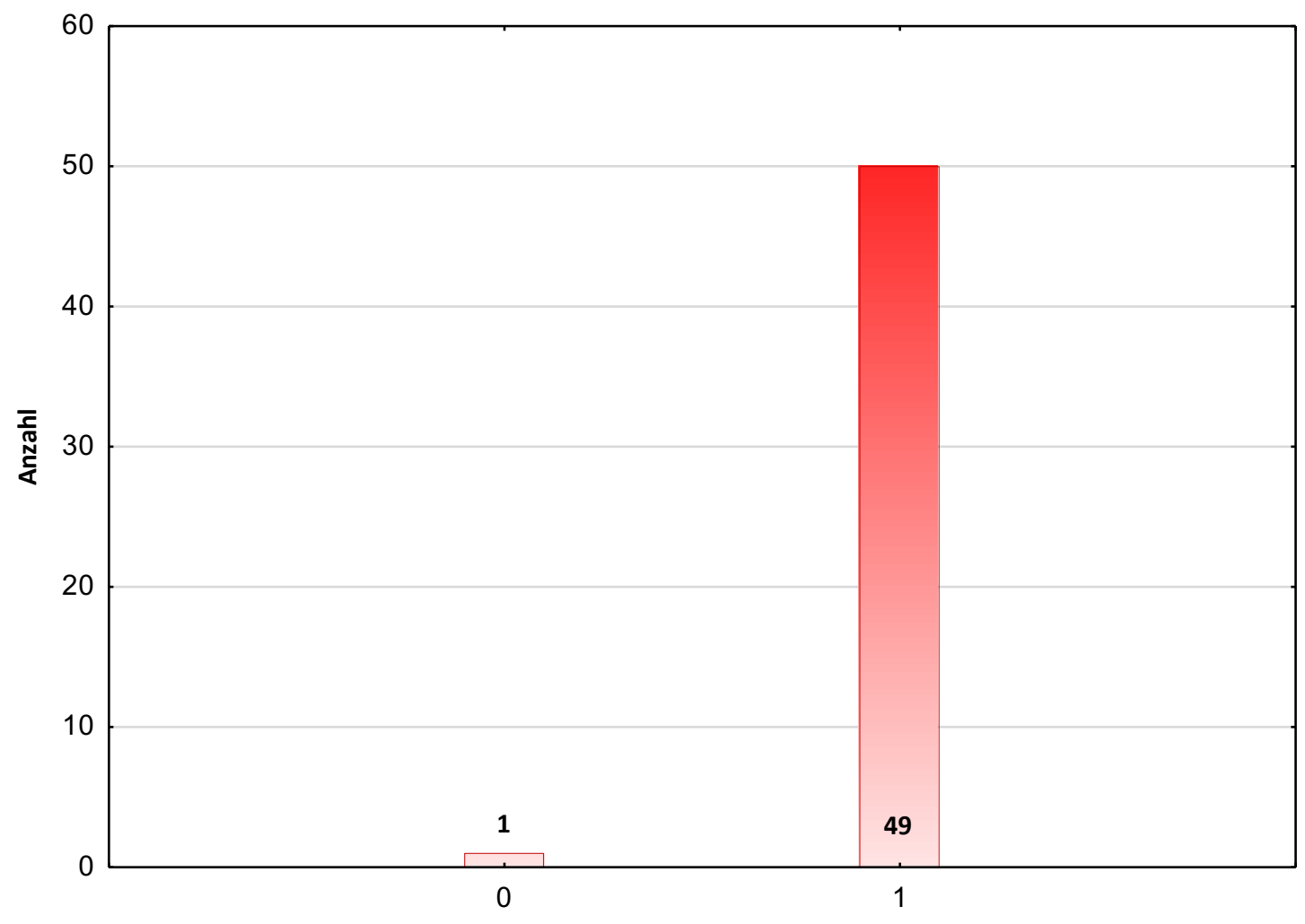

Ablaufzufriedenheit Fall 51-100

Abbildung 20: Zufriedenheit mit dem Ablauf des Operationstages nach Einführung des neuen Behandlungsplans $(0=$ nein, $1=\mathrm{ja})$.

Einige Patienten bekamen präoperativ ein kurz wirksames Benzodiazipin zur Anxiolyse. In dreizehn Fällen wurde vor der Operation gewichtsadaptiert Midazolam verabreicht. In allen dreizehn Fällen hatte die präoperative Verabreichung von Midazolam keinen Einfluss auf die postoperative Liegedauer im Aufwachraum (in beiden Fällen ergibt sich kein signifikanter Zusammenhang mit $\mathrm{p}>0,05)$.

\subsubsection{Perioperatives Management}

Die postoperative Zufriedenheit der Eltern mit der Versorgung ihres Kindes im Krankenhaus war bei deutlich hoher Gesamtzufriedenheit in allen Fällen unabhängig vom Alter des Kindes. Es ließ sich kein signifikanter Zusammenhang feststellen ( $p=0,392968)$. 
Zur Evaluation und Bewertung der Qualität der präoperativen Aufklärungen wurde die allgemeine Zufriedenheit der Eltern mit der Krankenhausversorgung in Korrelation mit den Bewertungen der einzelnen präoperativen Aufklärungen gesetzt.

Hier zeigte die Aufklärung bezüglich möglicherweise auftretender Nachblutungen einen signifikanten Einfluss auf die postoperative Zufriedenheit $(\mathrm{p}=0,041703)$. Sowohl die Bewertung der Schmerzaufklärung $(p=0,717449)$ als auch der anästhesiologischen Aufklärung $(\mathrm{p}=0,302617)$ korrelierten nicht signifikant mit der postoperativen Zufriedenheit, was womöglich auf die ohnehin sehr hohe Zufriedenheit der Eltern zurückzuführen ist.

Ein weiteres Item des Fragebogens bezog sich auf den Umfang der Aufklärungen im Allgemeinen. Die befragten Eltern bewerteten hier folgende Aussage:

1. Item zur Bewertung des Aufklärungsumfangs:

„In Vorbereitung auf die Operation bätte ich mir eine detailliertere Aufklärung gewünscht."

Im Folgenden sind die Fälle vor- und nach der Änderung des perioperativen Managements separat dargestellt. Elf Eltern, die den zusätzlichen schriftlichen Behandlungsplan und die Informationsschrift noch nicht erhalten hatten, wünschten sich eine detailliertere Aufklärung und bewerteten die Aussage mit $5=$ trifft völlig zu ( $n=50) .30$ Elternteile hingegen stimmten mit $1=$ trifft gar nicht zu (s. Abbildung 21).

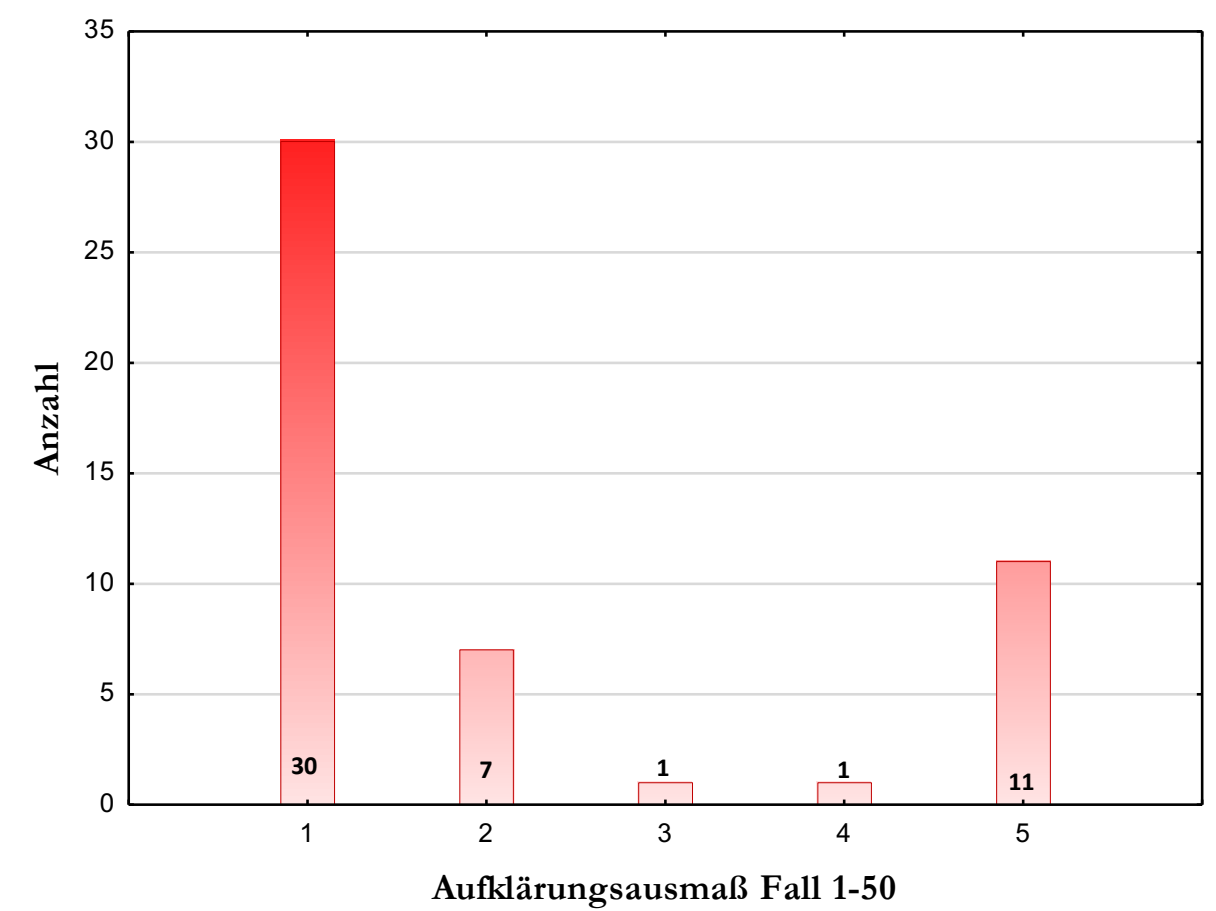

Abbildung 21: Zufriedenheit mit dem Ausmaß der Aufklärung vor Einführung des neuen Behandlungsplans $(1=$ trifft gar nicht $\mathrm{zu}, 2=$ trifft eher nicht $\mathrm{zu}, 3=$ unentschieden, $4=$ trifft eher zu, $5=$ trifft völlig zu). 
Nach Einführung des Behandlungsplanes und der Informationsschrift wurde dieselbe Aussage nur noch von fünf Eltern mit ,trifft völlig zu“ bewertet. 28 Eltern waren mit der Aufklärung zufrieden und wählten ,trifft gar nicht zu“, 16 Elternteile wählten die Antwortmöglichkeiten drei bis vier. Von einem Elternteil wurde die Frage nicht beantwortet (s. Abbildung 22).

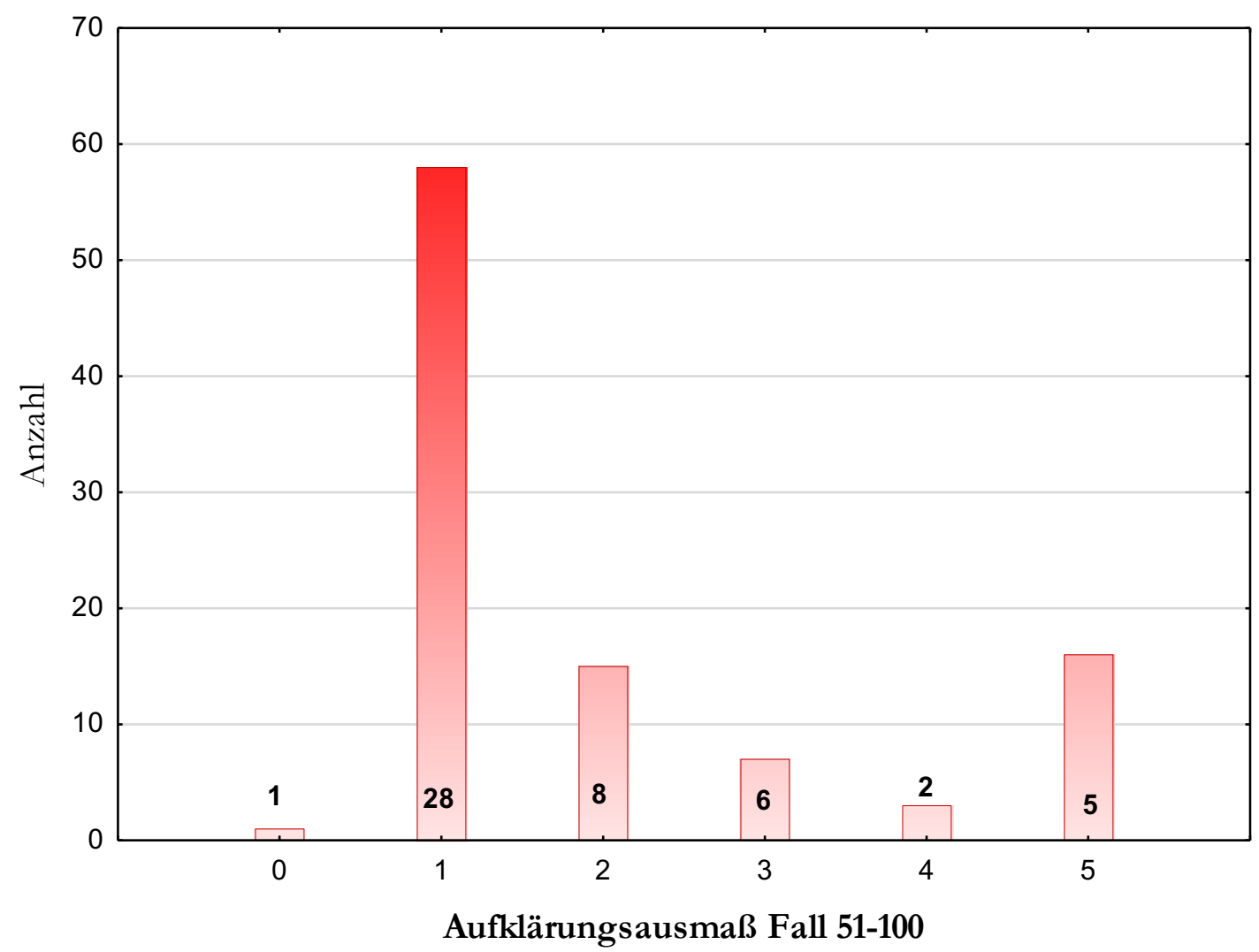

Abbildung 22: Zufriedenheit mit dem Ausmaß der Aufklärung nach Einführung des neuen Behandlungsplans $(1=$ trifft gar nicht zu, $2=$ trifft eher nicht zu, $3=$ unentschieden, $4=$ trifft eher zu, $5=$ trifft völlig zu). 
Die Zufriedenheit mit der Versorgung des Kindes im Krankenhaus wurde ebenfalls sowohl vor als auch nach Einführung des Behandlungsplans studiert. Die entsprechende Aussage des Fragebogens lautete:

1. Item zur Bewertung der Versorgung im Krankenhaus:

„Ich bin mit der Versorgung meines Kindes im Krankenhaus zufrieden. “

43 Eltern $(n=50)$, die keinen schriftlichen Behandlungsplan erhalten hatten, bewerteten das Item mit trifft völlig zu (=5). Vier Elternteile wählten die Antwortmöglichkeit „trifft eher $z u^{\prime \prime}$, drei wiederum entschieden sich für „unentschieden“. Im Vergleich hierzu bewerteten bereits 45 Elternteile nach Erhalt des Behandlungsplan die identische Aussage mit trifft völlig zu, zwei mit „trifft eher zu“ und drei mit „unentschieden“ (s. Abbildung 23, 24).

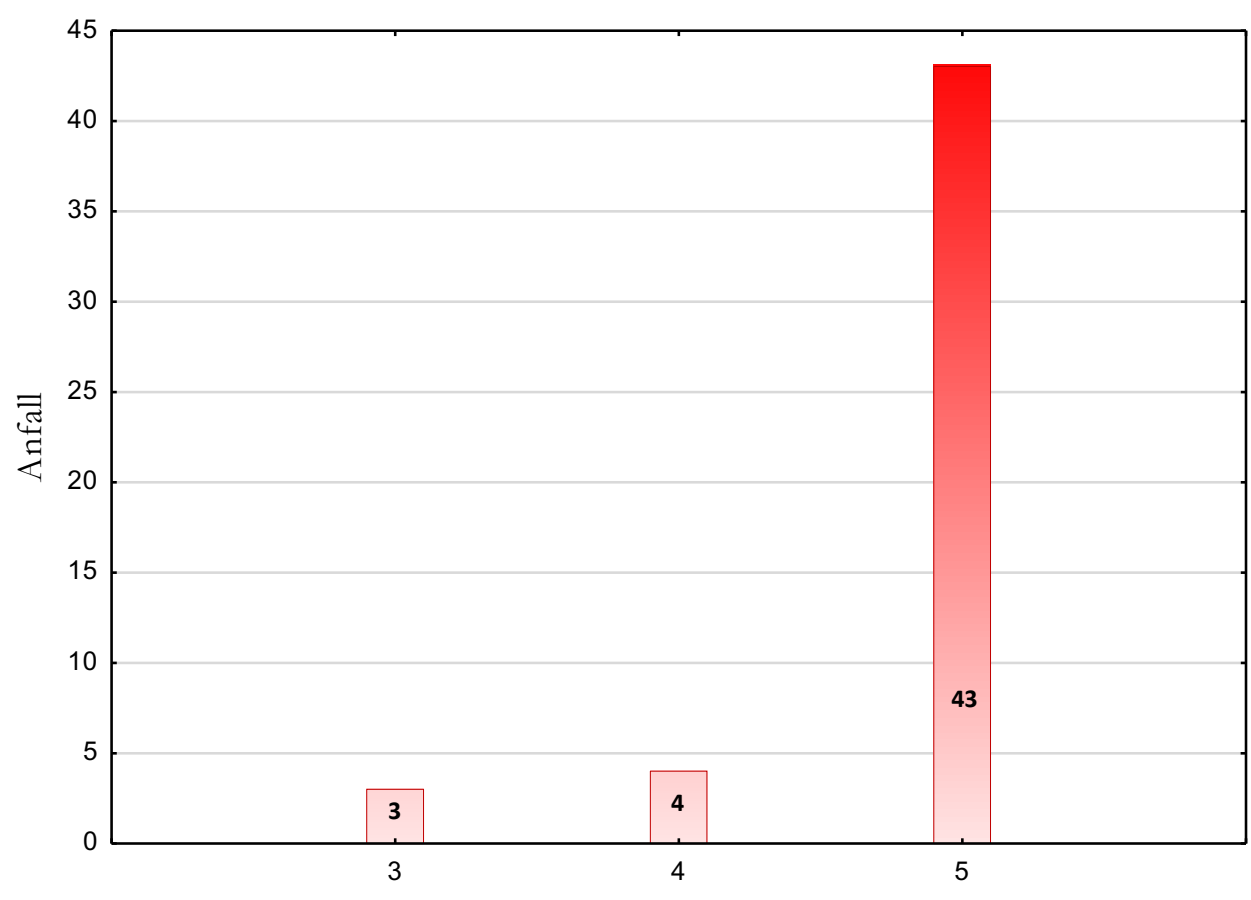

Grad der Zufriedenheit Fall 1-50

Abbildung 23: Zufriedenheit mit der Versorgung des Kindes im Krankenhaus vor Einführung des neuen Behandlungsplans $(1=$ trifft gar nicht zu, $2=$ trifft eher nicht zu, $3=$ unentschieden, $4=$ trifft eher zu, $5=$ trifft völlig zu). 


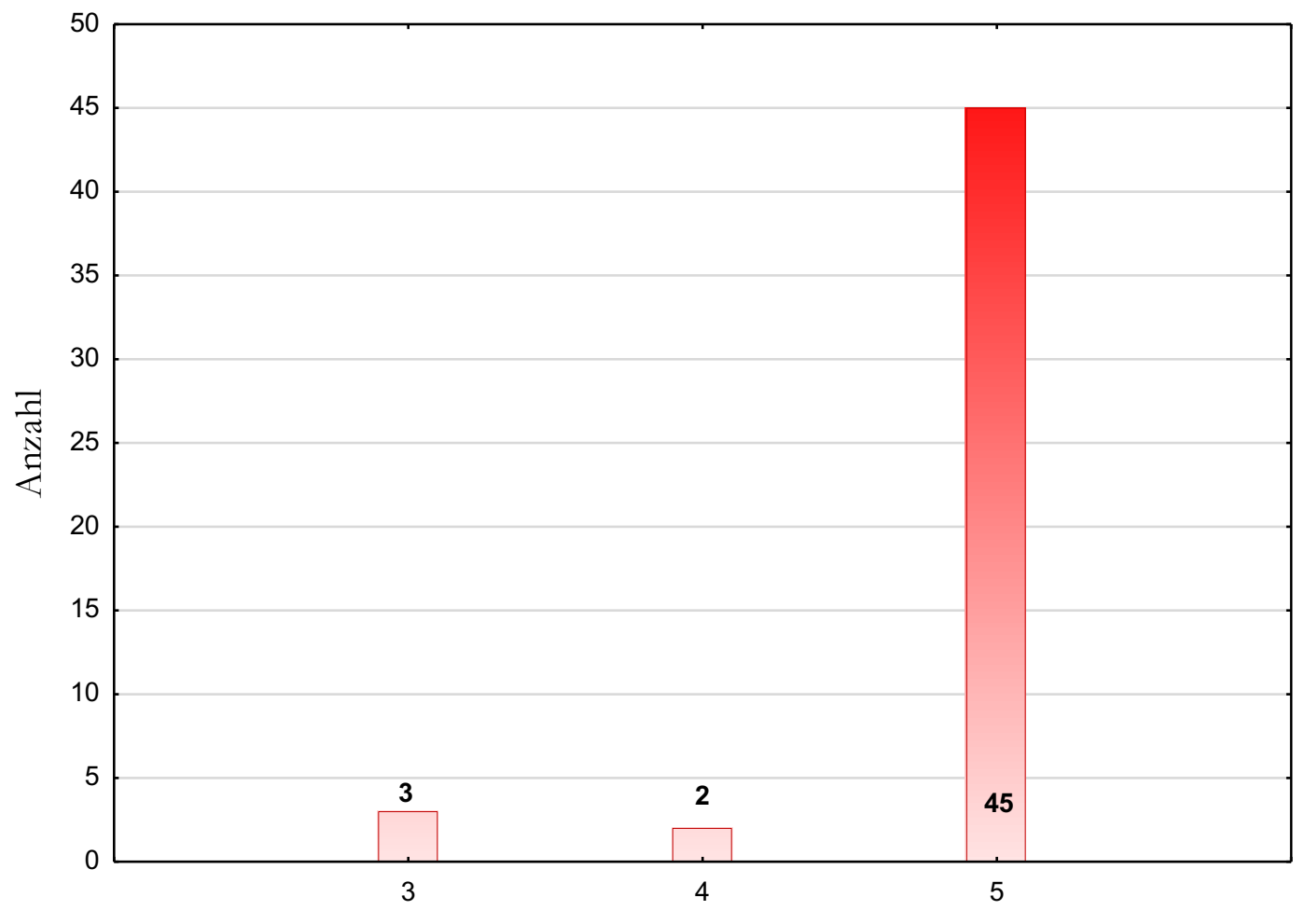

Grad der Zufriedenheit Fall 51-100

Abbildung 24: Zufriedenheit mit der Versorgung des Kindes im Krankenhaus nach Einführung des Behandlungsplans (1= trifft gar nicht $\mathrm{zu}, 2=$ trifft eher nicht $\mathrm{zu}, 3=$ unentschieden, $4=$ trifft eher zu, $5=$ trifft völlig zu). 


\section{Diskussion}

Im folgenden Teil der Dissertation werden die Studienergebnisse analysiert und kritisch bewertet und mit der Literatur verglichen. Hierbei wird zum einen auf die Qualität des Patientenkollektives zum anderen auf die einzelnen, im Ergebnisteil beschriebenen, Bestandteile der Patientenbefragung unter Berücksichtigung der Auswirkungen des neuen Behandlungskonzeptes näher eingegangen.

\subsection{Aussagefähigkeit des Patientenkollektivs}

Um die gesamte Studie besser bewerten zu können, wird zunächst die Auswahl des Patientenkollektivs kritisch beleuchtet.

Das gewählte Kollektiv ist mit einer Probandenzahl von 100 Patienten/innen, die über einen Zeitraum von 11 Monaten im Rahmen der Studie interviewt wurden, relativ klein. Ein prolongierter Studienverlauf hätte aufgrund des daraus resultierenden größeren Patientenkollektives eine stärkere Aussagekraft der Ergebnisse zur Folge gehabt.

Die Geschlechterverteilung ist mit 92 männlichen Patienten und nur 8 weiblichen Probandinnen unausgeglichen. Dies ist in vergleichbaren Studien ebenfalls der Fall und auf den gewählten chirurgischen Schwerpunkt zurück zu führen. Häufige Konsultationsgründe in der ambulanten Kinderchirurgie sind in dem Alter dem Fachgebiet der Urologie zuzuordnen. Einen überwiegenden Anteil dieser Krankheitsbilder bilden Pathologien der männlichen Geschlechtsorgane und Leistenhernien (Stein et al. 2017). Letztere treten vor allem bei männlichen Säuglingen und Kindern auf (Maddox und Smith 2008). Der Anteil der männlichen Probanden mit 92 Patienten ist so nicht verwunderlich und war zu erwarten.

Das durchschnittliche Alter der Mädchen betrug $6 \pm$ 0,85 Jahre, das der Jungen 4,8 \pm 0,3 Jahre. Viele Eltern gaben Schwierigkeiten bei der korrekten Einschätzung der Symptome ihrer Kinder an. Eine Befragung der Kinder persönlich, zum Beispiel unter Einbezug einer symbolischen Ratingskala, hätte bei einigen Fragen zu genaueren Angaben geführt.

Weiterhin könnte der Zeitpunkt der Datenerfassung ein Problem darstellen. Der vorliegende Datensatz wurde zu zwei Dritteln (mit Ausnahme der Fragebögen, die präoperativ beantwortete wurden) retrospektiv erfasst. In allen evaluierten Fällen verliefen die durchgeführten Operationen komplikationslos und die Kinder konnten postoperativ nach Hause entlassen werden. Zu diesem Zeitpunkt bewerteten die Eltern die präoperative Vorbereitung möglicherweise positiver, als sie es vor der Operation noch getan hätten.

Außerdem erfolgte die Befragung der Angehörigen telefonisch und nicht mit Hilfe eines schriftlichen Fragebogens. Einige Items erforderten eine Bewertung anhand einer LikertSkala oder anhand numerischer Skalen. Aufgrund der fehlenden Möglichkeit zur Visualisierung dieser Skalen, stellte sich die Bewertung der entsprechenden Aussagen häufig als schwierig heraus. Obendrein war die Kommunikation in einigen Fällen durch sprachliche 
Verständnisprobleme zusätzlich erschwert, was nicht immer eine exakte Datenerfassung möglich gemacht hat. Es erfolgte außerdem lediglich die telefonische Befragung eines einzelnen Elternteils, der möglicherweise abweichende Eindruck des Elternteils, welches nicht interviewt wurde, wurde bei der Datenerfassung dementsprechend nicht berücksichtigt.

Zu den evaluierten Aspekten gehörte unter anderem die Zufriedenheit der Eltern mit dem perioperativen Management. Der Begriff der Zufriedenheit wird durch verschiedenste Faktoren beeinflusst. Die verwendeten Fragebögen beinhalteten hierzu ausschließlich ein einziges Item, was möglicherweise nicht ausreicht, um so ein komplexes psychologisches Konstrukt adäquat und effizient zu bewerten.

\subsection{Interpretation und Fehleranalyse der Ergebnisse}

\subsubsection{Operationstechnische Daten}

Vor allem in der Kinderchirurgie wird ergänzend zu einer Allgemeinnarkose oft eine Regionalanästhesie gewählt, um perioperativ die Schmerzen zu reduzieren. Auch in der vorliegenden Studie wurde bei 69 von 100 Kindern zusätzlich eine Regionalanästhesie in Form eines Peniswurzelblockes oder einer Kaudalanästhesie verwendet. Die berechneten Korrelationen zeigten allerdings keinen signifikanten Zusammenhang mit der postoperativ angegebenen Schmerzsituation. Dies steht im Gegensatz zur aktuellen Studienlage.

Viele Studien belegen, dass gerade in der ambulanten Kinderchirurgie durch den additiven Gebrauch von Regionalanästhesien das postoperative schmerzfreie Intervall verlängert und der Gebrauch von Opioiden nachweislich reduziert werden kann (Beyaz et al. 2011). Gerade bei Eingriffen im Inguinal- und Genitalbereich wird sowohl die Kaudalanästhesie als auch der Peniswurzelblock regelmäßig verwendet, um eine effektive Schmerzreduktion zu erreichen und auch die ersten postoperativen Stunden analgetisch mit abzudecken. Dies hat nicht nur eine Verkürzung der Hospitalisierungszeit zur Folge, sondern bei adäquater Schmerzkontrolle auch eine gesteigerte Zufriedenheit von Seiten der Kinder und der Angehörigen. Zusätzlich handelt es sich bei diesen Formen der Regionalanästhesie gerade bei Kindern um komplikationsarme Verfahren (Kaya et al. 2012).

Die abweichenden Ergebnisse unserer Studie, also die fehlende Korrelation der durchgeführten Regionalanästhesie mit der postoperativen Schmerzsituation, sind womöglich auf die bereits erwähnten Schwierigkeiten bei der Einschätzung der Schmerzsituation durch die Eltern zurückzuführen. Außerdem wird der direkte Vergleich einer Regionalanästhesie mit der alleinigen Allgemeinnarkose durch die geringe Fallzahl der Kinder ohne Regionalanästhesie (31 Patienten von $n=100$ ) erschwert.

Ziel einer ambulant durchgeführten Operation sollte eine möglichst geringe Hospitalisierungszeit sein. Auch vor diesem Hintergrund wurde der Einfluss von 
kurzwirksamen Benzodiazepinen auf die postoperative Verweildauer im Aufwachraum untersucht.

Midazolam gilt als ein effektives Mittel zur präoperativen Anxiolyse bei Kindern. Zusätzlich begünstigt es eine latente anterograde Amnesie, was die Gefahr traumatischer Erinnerungen an die Narkoseeinleitung reduziert (Brosius und Bannister 2002). Die Narkoseeinleitung kann durch eine adäquate Prämedikation deutlich vereinfacht und beschleunigt werden. Laut verschiedener Studien hat die präoperative Gabe von Midazolam, unabhängig von der Dosierung, keinen Einfluss auf die postoperative Aufenthaltsdauer im Aufwachraum (Deshmukh et al. 2016).

In der vorliegenden Studie bekamen von hundert Kindern nur 24 präoperativ ein kurzwirksames Benzodiazepin zur Anxiolyse. In allen 24 Fällen wurde das Präparat Midazolam entweder in einer Dosierung von $5 \mathrm{mg}$, oder $10 \mathrm{mg}$ verabreicht. Im Einklang mit der aktuellen Studienlage zeigte sich auch hier kein signifikanter Zusammenhang mit der postoperativen Verweildauer im Aufwachraum und somit keine Verlängerung der Hospitalisierungszeit.

Einige Kinder wurden vor dem durchgeführten Eingriff bereits schon einmal einer Operation unterzogen. Für die überwiegende Mehrheit (81 von 100 teilnehmenden Kindern) war es allerdings die erste Operation. Dieser Aspekt korrelierte signifikant mit der Zufriedenheit der Eltern mit der Behandlung ihres Kindes im Krankenhaus. Der Teil der Eltern, deren Kind das erste Mal operiert wurde, waren im Schnitt deutlich zufriedener mit der Behandlung des Kindes. Die Eltern hingegen, deren Kind bereits in der Vergangenheit einem Eingriff unterzogen wurden, zeigten sich zum Teil unzufriedener. Ellerton et al wiesen in einer Studie daraufhin, dass Eltern deren Kinder bereits einmal operiert wurden, deutlich größere präoperative Ängste entwickelten als Eltern für deren Kind es die erste Operation war (Ellerton und Merriam 1994). Die vermehrte Unzufriedenheit und das erhöhte Angstlevel präoperativ sind vermutlich auf die Erfahrungen der Kinder und der Eltern in der Vergangenheit zurück zu führen. Es liegen keine aktuellen Studien vor, die die allgemeine Zufriedenheit der Eltern mit der Versorgung des Kindes im Krankenhaus mit dem Aspekt der Voroperation korrelieren und diskutieren. Eine Operation kann mit postoperativen Schmerzen und Unwohlsein einhergehen. Haben ein Kind und seine Eltern diese Erfahrungen bereits gemacht, sehen sie dem Tag der Operation möglicherweise mit vermehrten Ängsten entgegen. Diese Ängste beeinflussen die postoperative Zufriedenheit. Außerdem werden erneut aufgetretene postoperative Symptome wie Schmerzen oder Übelkeit unter Umständen negativer bewertet, da sie bereits das zweite Mal auftreten. Weiterhin wird die Versorgung des Kindes womöglich mit anderen Krankenhäusern und medizinischen Erfahrungen verglichen. Dieser Vergleichswert hat dementsprechend Einfluss auf die aktuelle Bewertung der Eltern.

Detailliertere Informationen bezüglich der Voroperation hätten eine präzisere Interpretation der Korrelation möglich gemacht. Ein komplikationsbehafteter Verlauf des ersten Eingriffes 
beeinflusst die Zufriedenheit der Eltern anders, als eine unkomplizierte Operation oder postoperative Beschwerden. Ebenfalls die Größe des Eingriffes, sowie die zuständige Abteilung und die durchführende Klinik wären für eine aussagekräftigere Korrelation vorteilhaft gewesen.

\subsection{Einfluss des neuen Behandlungskonzeptes}

Nach der vollständigen Befragung von den ersten 50 Eltern erfolgte die Einführung des bereits in 1.6 beschriebenen festgelegten Behandlungsplanes. Einige Aspekte wurden sowohl vor als auch nach der Einführung dieses Konzeptes untersucht. Es sollte speziell der Einfluss des Behandlungsplanes auf die Schmerzmittelgabe durch die Eltern, die Aufklärungsqualität (im Sinne einer zufriedenstellenden Vollständigkeit), den Tagesablauf, inklusive Zeitmanagement und Struktur des Operationstages, die allgemeine Versorgungszufriedenheit im Krankenhaus und zu Hause und die perioperativen Sorgen und Ängste seitens der Eltern evaluiert werden. Im Folgenden werden entsprechend der untersuchten Themenkomplexe die Ergebnisse diskutiert und vor dem Hintergrund der aktuellen Literatur beleuchtet.

\subsubsection{Postoperative Symptomkomplexe}

Operative Eingriffe sind nahezu immer mit auftretenden Symptomen verbunden, die für den Patienten oft unangenehm sind. Schmerzen, Schwellungen, komplizierte Wundheilungsverläufe, aber auch Übelkeit und Erbrechen sind häufige Beschwerden, die nach einer Operation auftreten können. Gerade die postoperativen Schmerzen sind wiederholt Inhalte diverser Studien, mit dem Ziel diese möglichst zu minimieren und zu kontrollieren.

Von der ersten Kohorte der Studie, also jene Patienten und Elternteile, die lediglich die mündliche Aufklärung und keinen schriftlichen Behandlungsplan erhielten, gaben 41 Eltern $(\mathrm{n}=50)$ ihrem Kind am Operationstag zu Hause ein Schmerzmedikament. Die zweite Kohorte umfasste die Eltern und Patienten, die zusätzlich zur mündlichen Aufklärung noch einen schriftlichen Bogen, der Uhrzeit und Medikation der nächsten Schmerzmittelgabe enthielten. Retrospektiv konnte eine effektive Steigerung der abendlichen Schmerzmittelgabe auf 49 Elternteile festgestellt werden.

Das postoperative Schmerzmanagement ist in der aktuellen Literatur ein weit verbreitetes Thema. Viele Kinder haben nach einer Operation noch moderate bis starke Schmerzen. Ein noch häufig auftretendes Problem ist weiterhin die Unterversorgung der Kinder mit Schmerzmitteln in der häuslichen Umgebung (Paquette et al. 2013; Rony et al. 2010). Diverse Studien beschäftigen sich mit möglichen Ursachen dieser Problematik. Als beeinflussende Faktoren werden unter anderem eine fehlerhafte Einschätzungen der Nebenwirkungen, Zweifel an den Dosierungsanweisungen, die Angst vor Abhängigkeit und Gewöhnung sowie 
die Akzeptanz von postoperativen Schmerzen als notwendiges Übel aufgeführt (Kankkunen et al. 2003; Forward et al. 1996; Hamers und Abu-Saad 2002).

Die Verantwortung eines adäquaten Schmerzmanagements wird bei ambulant durchgeführten Operationen auf die Eltern übertragen. Damit die Eltern dieser Verantwortung auch gerecht werden können, ist eine effiziente Vorbereitung und Unterstützung durch das medizinische Personal von Nöten. Für ein erfolgreichen Umgang mit den Schmerzen des Kindes zu Hause, müssen die Eltern nicht nur die Schmerzen des Kindes und eine ausreichende Medikamentendosis einschätzen können, sondern auch außer medikamentöse Strategien, wie liebevolle Zuwendung und spielerische Ablenkung, entwickeln. (MacLaren Chorney et al. 2014).

In der Vergangenheit wurden bereits diverse Strategien zur suffizienten Vorbereitung der Eltern auf die Schmerzsituation ihrer Kinder zu Hause untersucht. Der Ansatz dieser Studie, die Medikation und die Dosierung schriftlich festzulegen, konnte die Schmerzmittelgabe durch die Eltern wie oben beschrieben auf $98 \%$ steigern.

Hegarty (2013) hingegen konnten mit ihrer im Mai 2013 veröffentlichten Studie keinen überlegenen Effekt einer kombinierten schriftlichen und verbalen Aufklärung feststellen. Die Informationen waren von den Eltern häufig nicht erinnerlich, Auswirkungen auf die Schmerzmittelgabe durch die Eltern konnten nicht evaluiert werden. Dies wurde auf eine mögliche Überladung mit Informationen zurückgeführt.

Longard und Coautoren beschrieben eine ähnliche Problematik. Die Eltern bewerteten die präoperativen Aufklärungen bezüglich eventuell auftretender Schmerzen durch aus als sehr positiv. Dennoch schien die Aufklärung ineffizient, da trotz allem postoperativ Schwierigkeiten bei der Versorgung der Kinder mit Schmerzmitteln auftraten. Auch hier wurden Ängste von Seiten der Eltern bezüglich möglicher Überdosierungen und Nebenwirkungen, aber auch die grundsätzliche Haltung der Eltern gegenüber Medikamenten als Ursache diskutiert und vermutet (Longard et al. 2016).

Auch Versuche mit visuellen Analogskalen zur Schmerzevaluation für die Kinder zu Hause zeigten keinen positiven Einfluss auf die ambulante postopertaive Schmerzmittelgabe im Vergleich zum „normalen“ Aufklärungsgespräch (Unsworth et al. 2007).

Um mögliche Ursachen der insuffizienten Schmerztherapie in der häuslichen Umgebung herauszufiltern und effiziente Lösungsstrategien zu entwickeln, wurde von allen oben genannten Autoren die weiterführende Forschung zu diesem Thema dringlich empfohlen.

Neben den Schmerzen können nach einer Operation aber auch andere Symptome auftreten.

$\mathrm{Zu}$ der Frage in welchem Maße bestimmte Symptome die Eltern- bzw. Patientenzufriedenheit beeinflussen, gibt es unterschiedliche Thesen in der aktuellen Studienlage. Einige Studien stellten durchaus eine Korrelation zwischen den Schmerzen des Kindes und der Elternzufriedenheit her. Eltern, deren Kinder eine adäquates Schmerzmanagement erhielten, waren hoch signifikant zufriedener $(p<0,001)$ mit anderen 
Aspekten des perioperativen Managements, wie zum Beispiel der medizinischen Versorgung und der Aufklärung durch das Personal (Chan et al. 1998; Ygge und Arnetz 2001).

Eine 2016 durchgeführte retrospektive Studie aus den USA wiederum konnte keinen signifikanten Zusammenhang zwischen aufgetretenden postoperativen Komplikationen und der erlebten Zufrieden- bzw. Unzufriedenheit mit der Versorgung im Krankenhaus feststellen. Als Ursache für ihre Unzufriedenheit gaben die Befragten hier eher organisatorische Aspekte, wie zum Beispiel Wartezeiten, an (Brenn et al. 2016).

Auch in dieser Studie ließ sich kein Zusammenhang zwischen postoperativ aufgetretenen Symptomen, unter anderem auch Schmerzen, mit der Zufriedenheit der Eltern feststellen $(p>0,05)$. Beeinflusst wurden diese Berechnungen eventuell durch das hohe Maß an Zufriedenheit bei nahezu allen befragten Elternteilen. Bei 100 teilnehmenden Probanden wurde die Aussage „Ich bin mit der Versorgung meines Kindes im Krankenhaus zufrieden“ kein Mal mit „trifft gar nicht zu“, oder „trifft eher nicht zu“ bewertet. Unabhängig von den vermuteten Schmerzen oder weiteren zuvor bereits angesprochenen Symptomen war die Zufriedenheit mit der Krankenhausversorgung im gesamten Kollektiv sehr hoch.

Obwohl die Schmerzen der Kinder signifikant mit der Zeit bis zur absoluten Beschwerdefreiheit korrelierten $(p<0.05)$, zeigte auch dieses zeitliche Intervall keinerlei Einfluss auf die Zufriedenheit der Eltern mit der Versorgung ihres Kindes. Möglicherweise ist dies ebenfalls mit der hohen Gesamtzufriedenheit zu begründen.

Im Allgemeinen zeigten die Kinder nach den Operationen wenig Symptome, lediglich 11 Kinder beklagten Übelkeit, nur 6 von ihnen mussten sich zusätzlich übergeben. In 36 Fällen wurde eine Schwellung des Operationsgebietes ohne Auswirkungen auf die Befindlichkeit des Kindes $(p>0.05)$ angegeben. Dies entspricht den medizinischen Erwartungen an die postoperativen Verläufe, da es sich bei den durchgeführten Operationen um kleinere urologische Eingriffe handelt. Diese verlaufen in der Regel komplikationsarm und sind auch gerade deswegen gut für eine ambulante Durchführung geeignet (Stein et al. 2017). Der postoperative telefonische Kontakt mit den Eltern und ihre Angaben bezüglich der beim Kind registrierten Symptome bestätigen diese Annahme.

\subsubsection{Organisatorische Parameter}

Um die Organisationsstruktur des Operationstages genau zu evaluieren, wurde nicht nur die allgemeine Zufriedenheit der Eltern mit der Versorgung des Kindes im Krankenhaus erfragt, sondern zusätzlich auf die Zufriedenheit mit der Struktur und dem Ablauf des Tages eingegangen. Hierbei sollte die Vorhersehbarkeit des Operationstages in seiner Struktur und der Ablauf des Tages selbst bewertet werden.

Auch in diversen Studien wird der organisierte, strukturierte Ablauf des Operationstages als Einflussfaktor auf die postoperative Zufriedenheit der Eltern aufgeführt. Laut Literatur beeinflussen einzelne Aspekte, wie Wartezeiten und flüssige Übergänge der einzelnen 
Behandlungsschritte, die Zufriedenheit der Eltern mit dem perioperativen Management deutlich mehr als postoperative Komplikationen. Dies wird unter anderem mit der Erwartungshaltung der Eltern begründet. Häufig werden kleinere Komplikationen bzw. Symptome, wie Schmerzen oder Übelkeit, nach einer Operation erwartet. Faktoren die von eben diesen präoperativen Erwartungen abweichen, wie unvorhergesehene Ereignisse oder verlängerte Wartezeiten, steigern die Unzufriedenheit von Seiten der Eltern (Brenn et al. 2016).

Im Einklang mit der Literatur korrelierte nach unseren Berechnungen die Zufriedenheit mit dem Ablauf des Operationstages signifikant mit der Zufriedenheit der Versorgung im Krankhaus $(\mathrm{p}<0,005)$. Dies betont noch einmal die Wichtigkeit des reibungslosen und transparenten Ablaufes für die Gesamtzufriedenheit der Eltern und der Patienten.

\subsubsection{Perioperatives Management}

Ein wichtiger Bestandteil des perioperativen Managements ist die präoperative Vorbereitung und Aufklärung. Inhalte dieser Aufklärungen sollten die verständliche und transparente Beschreibung des Operationsablaufes mit möglichen Komplikationen, die Narkoseaufklärung und die Vorbereitung auf postoperativ auftretende Schmerzen sein. Die Aufklärungen erfolgen nicht nur mit dem Ziel der Informierung der Patienten und Eltern sowie der rechtlichen Absicherung des behandelnden Arztes, sondern auch um Ängste und Sorgen vor der Operation möglichst zu reduzieren.

Inwiefern eine adäquate Vorbereitung und Aufklärung die Ängste und auch die Zufriedenheit mit dem Operationstag beeinflusst, wurde bereits in einigen Studien untersucht. Hauptsächlich wird hier auf die anästhesiologische Aufklärung und ein effizientes perioperatives Schmerzmanagement eingegangen.

Spencer und Franck betonten in einer 2005 durchgeführten Studie die Verantwortung der Eltern für die Gesundheit ihres Kindes. Sie müssten Entscheidungen treffen, die im optimalen Fall auf einer Basis eines ausreichenden Kenntnisstandes gefällt werden. Vor diesem Hintergrund sei eine adäquate Aufklärung mit samt der Risikofaktoren unerlässlich. Die Autoren zeigten in diesem Zusammenhang einen Wissenszuwachs der Eltern nach mündlicher Aufklärung sowie Erhalt einer Informationsbroschüre (Spencer und Franck 2005).

Eine 2011 in Australien durchgeführte retrospektive Studie zeigte außerdem, dass die präoperative Vorbereitung eine signifikante Reduktion der Ängste von Seiten der Eltern $(p<0,009)$ zur Folge hatte, ohne jedoch einen anxiolytischen Effekt auf die Kinder zu haben $(\mathrm{p}<0,07)$. Untersucht wurden hier 73 Kinder und jeweils ein Elternteil.

Eine direkte Korrelation zwischen der Angst der Eltern und der Angst der Kinder konnte aber nachgewiesen werden, sodass eine ausführliche präoperative Vorbereitung der Eltern letztendlich doch eine positive Auswirkung auf die Befindlichkeit der Kinder hatte. Eine 
präoperative Angstreduktion bei den Kindern zeigte außerdem positive Effekte auf das postoperative Verhalten nach der Narkose. Negative Erfahrungen wie schlechte Träume oder Weinen beim Erwachen aus der Narkose sollten so erfolgreich verhindert werden (Fincher et al. 2012).

Eine kombinierte mündliche und schriftliche Information stellt anhand verschiedener Studien die beste Möglichkeit für eine angemessene Aufklärung dar. Die Eltern können so bei Bedarf die Informationsschrift wiederholt studieren (Andersson et al. 2015). Andere Studien sind diesbezüglich konträrer Meinung. Sie konnten keinen nachweislichen Wissenszuwachs der Eltern nach einer schriftlich formulierten Aufklärung evaluieren. Möglicherweise waren die Informationsblätter zu komplex und wurden nicht immer gelesen (Spencer und Franck 2005).

In unserer Studie herrschte eine sehr hohe Gesamtzufriedenheit mit der erfolgten Aufklärung (Risikofaktoren wie Nachblutungen, Aufklärung in der Anästhesiesprechstunde, Aufklärung bezüglich auftretender Schmerzen). Deutlich mehr als die Hälfte der befragten Eltern gaben an, vollständig aufgeklärt worden zu sein. Bei den nachfolgenden Berechnungen zeigte sich, dass eine signifikante Korrelation zwischen der Aufklärung über mögliche Risikofaktoren, speziell der Nachblutung, und der Zufriedenheit der Eltern festgestellt werden konnte. Die Aufklärung bezüglich auftretender Schmerzen und die Narkoseaufklärung allerdings zeigten keinen nachweislichen Effekt auf die postoperative Zufriedenheit der Eltern mit der Versorgung (jeweils $\mathrm{p}>0$,05). In der aktuellen Literatur findet man hierzu ebenfalls widersprüchliche Angaben. Zum Teil entspricht die Meinung unsere Studie und eine signifikante Korrelation zwischen einer ausführlichen Aufklärung und der postoperativen Zufriedenheit konnte nicht nachgewiesen werden (Fincher et al. 2012). Andere Studien wiederum stellten durchaus einen Zusammenhang her. Diese bezogen sich vor allem auf auftretende Schmerzen nach der Operation, die durch eine angemessene Aufklärung präoperativ nachweislich reduziert werden konnten. Dies hatte wiederum eine Steigerung der Zufriedenheit mit dem Operationstag zur Folge (Sjöling et al. 2003).

Ebenfalls im Rahmen des neuen Behandlungsplans wurde die präoperative Aufklärung hinsichtlich ihrer Vollständigkeit überprüft und auch hier der Einfluss des schriftlichen Informationsbogens evaluiert. Bestandteil der präoperativen Vorbereitung sollten eine transparente Beschreibung der Struktur des Operationstages und die Vermittlung bestimmter Verhaltensparameter, wie die Nahrungs- und Trinkkarenz präoperativ, der Zeitpunkt für den Pflasterwechsel, Empfehlungen bezüglich postoperativer Belastung und Mobilisation, sowie Angaben zur Schmerzmittelgabe sein.

Schon die erste Fallgruppe (erhielt keinen Bogen) war mit der präoperativen Aufklärung sehr zufrieden. Nur 11 Elternteile ( $\mathrm{n}=50)$ wünschten sich eine detailliertere Aufklärung. Von der zweiten Fallgruppe, die den schriftlichen Behandlungsbogen erhielt, forderten allerdings nur noch fünf Elternteile ( $\mathrm{n}=50$ ) eine detailliertere Vorbereitung vor der Operation. 
Allerdings zeigte sich in beiden Fallgruppen ein guter Kenntnisstand der Eltern bezüglich der präoperativen Nüchternperiode (84 Elternteile gaben an, den Zeitraum der Nüchtern Periode zu kennen) und des Zeitpunktes für den Pflasterwechsel (100 von 100 Eltern kennen den postoperativen Tag für den Pflasterwechsel), und dies unabhängig von dem Medium der Aufklärung.

Auch Landier et al. (2017) evaluierten in einer aktuellen Studie den Einfluss einer Informationsbroschüre auf die postoperative Zufriedenheit, auf Ängste von Seiten der Eltern und deren Kenntnisstand über vermittelte Informationen. 178 Patienten, unterteilt in zwei Gruppen, die eine mit- die andere ohne zusätzliche Broschüre, bewerteten die oben genannten Parameter nach der präoperativen Aufklärung und am Tag der Operation. Landier und Koautoren (2017) konnten einen signifikanten Einfluss der schriftlichen Informationsvermittlung auf die postoperative Zufriedenheit, das faktische Wissen der Eltern und eine signifikante Angstreduktion nachweisen.

Kritisch anzumerken ist bei unserer Studie die allgemeine Formulierung des gewählten Items. Die Eltern bewerteten die Aussage: ,in Vorbereitung auf die Operation hätte ich mir eine detaillierte Aufklärung gewünscht“. Der Begriff „Aufklärung“ ist hier nicht eindeutig definiert und daher nicht trennscharf genug. Trotzdem zeigt sich durch die schriftliche Fixierung der zusätzlich verbal kommunizierten Empfehlung eine Reduzierung der ohnehin geringen Unzufriedenheit mit der präoperativen Vorbereitung.

Im Rahmen der Einführung des Behandlungsplanes wurde außerdem der Einfluss auf die allgemeine Zufriedenheit mit der Versorgung des Kindes im Krankenhaus untersucht. Auch hier konnte ein relevanter Zuwachs der Zufriedenheit (zwei zusätzliche Patienten bewerteten nach der Einführung des Planes die Aussage: „Ich bin mit der Versorgung meines Kindes im Krankenhaus zufrieden“ mit „trifft völlig zu“) eruiert werden. Außerdem ist zu erwähnen, dass bei allen Patienten unabhängig vom schriftlichen Behandlungsplan eine hohe Gesamtzufriedenheit evaluiert wurde. Kein Elternteil äußerte sich unzufrieden mit der Versorgung des Kindes im Krankenhaus. Lediglich sechs $(n=100)$ Elternteile wählten die Antwortmöglichkeit „unentschieden“ zur Bewertung des Items bezüglich der Versorgungszufriedenheit.

Diese hohe allgemeine Zufriedenheit entspricht den Ergebnissen verschiedener anderer Studie. Auch hier waren nahezu alle Patienten mit dem perioperativen Management zufrieden (Sjöling et al. 2003; Comley und DeMeyer 2001). Zu der Frage inwieweit die Patientenzufriedenheit durch schriftliche Informationsbögen beeinflussbar ist, finden sich in der Literatur gegensätzliche Ergebnisse. In einer 2003 veröffentlichten Studie konnten Sjöling und Koautoren einen signifikanten Zusammenhang zwischen der postoperativen Zufriedenheit mit dem perioperativen Mangement und der präoperativen Kombination aus verbaler und schriftlicher Aufklärung feststellen (Sjöling et al. 2003). Auch Andersson et al beschrieb 2015 einen positiven Effekt der schriftlichen Informierung auf die postoperative Zufriedenheit. 
Im Gegensatz dazu konnten andere Studien keinerlei signifikante Zusammenhänge zwischen der Zufriedenheit der Eltern mit dem perioperativen Management und der schriftlichen Form der Informationsvermittlung erkennen (Hegarty et al. 2013; Longard et al. 2016). Auch aufgrund der bereits erwähnten Komplexität des Zufriedenheitsbegriffes erscheint die zukünftige Forschung zur weiteren Optimierung des perioperativen Managements und der damit verbundenen Elternzufriedenheit von großer Bedeutung (Longard et al. 2016).

Schwerpunkt verschiedener Studien ist auch die Reduktion der präoperativen Angst der Kinder. Diese hat wie bereits erwähnt nicht nur Auswirkungen auf postoperative Verhaltensweisen des Kindes, sondern auch auf die auftretenden Schmerzen (Miguel Romeo und Sagardoy Muniesa 2014). Der von uns verwendete Fragebogen zielte auf die postoperative Zufriedenheit ab und weniger auf präoperative Ängste von Seiten der Eltern und der Kinder. Es wurde vor allem auf die Qualität und den Umfang der Aufklärungen und Vorbereitungen eingegangen. Eine Studie mit validierten Fragen zum Thema präoperative Ängste ist obligatorisch und sollte ergänzend zu der hier durchgeführten Studie angestrebt werden. Nur wenn verschiedene Aspekte und Parameter der Operationsstruktur sowie des perioperativen Managements beleuchtet werden, kann dieses optimiert stattfinden und negativ beeinflussende Aspekte können minimiert werden. Die Verringerung solcher Einflüsse sollte das Ziel interagierender Forschung sein.

Die erfolgten Aufklärungen wurden vorab nicht standardisiert, was möglicherweise zu einem geringen Verlust der Trennschärfe der entsprechenden Items geführt hat. Je nach aufklärender Person war der Informationsfluss möglicherweise nicht genau identisch und der beruhigende Einfluss auf die Kinder und Eltern unterschiedlich. Da die präoperativen Aufklärungen aber nicht ausschließlich von einer einzigen Person und immer im identischen Wortlaut durchgeführt werden können, ist diese Einschränkung leider unvermeidbar.

Deutlich war auch die Korrelation der Ablaufzufriedenheit des Operationstages mit der allgemeinen Zufriedenheit der Eltern mit dem perioperativen Management. Wir konnten hier einen hoch signifikanten Zusammenhang feststellen $(\mathrm{p}<0,005)$.

Ziel war es den Ablauf des Operationstages durch die verteilten Informationsbroschüren noch zu verbessern. Die ohnehin schon hohe Zufriedenheit mit dem Tagesablauf (Fallgruppe ohne schriftlichen Behandlungsplan: 44 zufriedene Elternteile bei $n=50$ ) konnte durch den zusätzlichen schriftlichen Behandlungsplan noch gesteigert werden (Fallgruppe mit zusätzlichem Plan: 49 zufriedene Elternteile bei n=50).

In Zusammenschau der diskutierten Aspekte sollte der Tag der ambulanten Operation für die Kinder und ihre Eltern möglichst transparent und klar strukturiert ablaufen. In vorbereitenden Gesprächen sollten neben den medizinischen Aufklärungen über das gewählte Narkoseverfahren und mögliche Komplikationen nach der Operation, auch vorbereitende organisatorische Informationen wie Ort und Zeit der Vorstellung am Tag der Operation, Rahmendaten der Nüchtern Periode präoperativ und Verhaltensregeln für die Zeit nach dem Eingriff vermittelt werden. 
Den Eltern sollte bewusst sein, wo sie sich mit ihren Kindern am Operationstag einfinden sollen und vor Ort zeitnah vom Pflegepersonal in Empfang genommen werden.

Kurzfristige terminliche Absprachen seitens der Eltern mit der zuständigen operativen Abteilung können Wartezeiten am Tag der Operation minimieren und so die Zufriedenheit und Sicherheit der Eltern steigern.

Allerdings ist nicht nur die Zufriedenheit mit der Versorgung im Krankenhaus ein wichtiger Aspekt. Auf die perioperativen Ängste und deren Ursachen sollte in weiteren Studien noch intensiver eingegangen werden, um das perioperative Management weiter zu perfektionieren. Präoperative schriftliche Vorgaben bezüglich der postoperativen Schmerzmittelgabe mit Dosiserungen und zeitlicher Terminierung steigern nachweislich die Compliance der Eltern die Analgetika zu verabreichen und reduzieren so nachweislich die postoperativen Schmerzen der Kinder. 


\section{$5 \quad$ Zusammenfassung}

Das ambulante Operieren erlebt in den letzten Jahren einen stetigen Aufschwung. Auch in der Kinderchirurgie werden Operationen inzwischen gerne im ambulanten Setting durchgeführt. Ambulante Operationen und die damit verbundenen Krankenhausaufenthalte stellen, so kurz sie auch sein mögen, für Kinder und ihre Eltern eine potentiell stressige und anstrengende Situation da. Ein effizientes perioperatives Management ist enorm wichtig, um diesen Tag für die Patienten und ihre Eltern so erträglich wie nur eben möglich zu gestalten. Schlechte Erfahrungen des Kindes sollten vermieden, und die Eltern adäquat auf die Versorgung des Kindes in der häuslichen Umgebung vorbereitet werden.

In der vorliegenden Dissertation wird die Auswirkung eines neu eingeführten perioperativen Behandlungskonzeptes bei ambulant zu operierenden Kindern auf die erlebten prä- und postoperativen Stunden thematisiert. Hierzu wurden Eltern von 100 Kindern, die zwischen Oktober 2015 und November 2016 in der Universitätsmedizin Göttingen mit dem Schwerpunkt der Kinderchirurgie und Kinderurologie ambulant operiert wurden, sowohl schriftlich als auch telefonisch kontaktiert und befragt. Nach Evaluation der ersten fünfzig Kinder erfolgte die Einführung des neuen Behandlungskonzeptes und anschließend die Evaluation weiterer fünfzig Probanden. Ein besonderes Augenmerk wurde bei der Befragung auf die Zufriedenheit mit dem perioperativen Management und außerdem auf die Auswirkungen des neuen Behandlungskonzeptes gelegt. Ziel war es die Zufriedenheit und Sicherheit zu steigern, eine konsequente Schmerzmittelgabe zu bewirken, organisatorische Informationen zu vermitteln und die Struktur des Operationstages transparenter zu gestalten.

Die Elternzufriedenheit und eine adäquate Versorgung des Kindes nicht nur im Krankenhaus, sondern auch postoperativ zu Hause, sind häufig Inhalt aktueller Studien. Schwerpunkt wird hier meist auf das Schmerzmanagement gelegt, speziell auf die Unterversorgung mit Schmerzmitteln, welche trotz intensiver Forschungen und Bemühungen noch nicht hinreichend verhindert werden kann. Es wurden bereits Einflussfaktoren auf die Elternzufriedenheit extrahiert, wobei noch häufig Schwierigkeiten bei der Entwicklung von Lösungsstrategien beschrieben werden, sodass viele Autoren weiterführende Untersuchungen zur Optimierung des perioperativen Managements empfehlen.

Im Rahmen dieser Studie konnte eine signifikante Korrelation $(p<0,005)$ zwischen der Elternzufriedenheit mit der Versorgung des Kindes im Krankenhaus und der Zufriedenheit bezüglich des Operationstagesverlaufes nachgewiesen werden. Die Einführung des neuen Behandlungskonzeptes führte zu einer nachweislichen Steigerung der Zufriedenheit mit der Tagesstruktur, der allgemeinen Elternzufriedenheit mit dem perioperativen Management, sowie zu einer verlässlicheren postoperativen Schmerzmittelgabe im ambulanten Setting. 
Weitere Faktoren wie Alter, Geschlecht, Operation, Symptome wie Übelkeit, Erbrechen und Schmerzen, verwendete Anästhesieformen, die Atemwegssicherung, und verabreichte Medikamente wurden evaluiert und diskutiert. Es korrelierten weiterhin die angegebenen Schmerzen mit der Zeit bis zu Beschwerdefreiheit $(\mathrm{p}<0,05)$ und die Zufriedenheit der Eltern mit der Angabe bezüglich einer Voroperation $(p<0,05)$.

Kein signifikanter Zusammenhang konnte zwischen der Zufriedenheit der Eltern und der Qualität der präoperativen Aufklärungen bezüglich auftretender Schmerzen und der Qualität des Anästhesiegesprächs festgestellt werden.

Die Bewertung der Versorgung des Kindes im Krankenhaus war unabhängig von dem Alter des Kindes.

Die vorliegenden Ergebnisse müssen unter dem Vorbehalt eventueller methodischer Schwächen bewertet werden. Hierzu zählen die Evaluation von nur einem Elternteil ohne Berücksichtigung konträrer Meinungen des anderen Erziehungsberechtigten und die telefonische Evaluation mit zeitweiligen Kommunikationsschwierigkeiten aufgrund von Sprachbarrieren. Möglicherweise sind die Korrelationen durch das kleine Patientenkollektiv nur eingeschränkt beurteilbar.

Die größte Einschränkung der vorliegenden Arbeit ist die zu geringe Trennschärfe der einzelnen Items der Fragebögen. Gerade der Begriff der Zufriedenheit beschreibt ein multifaktorielles Konstrukt, welches detailliertere, psychologisch validierte Fragebögen erfordert.

Trotz der beschriebenen Einschränkungen wurde in unserer Studie durch das neue schriftlicher Behandlungskonzept die Zufriedenheit der Eltern mit dem perioperativen Management und auch die Compliance bezüglich empfohlener Behandlungsmaßnahmen erheblich gesteigert. Die Kombination von mündlicher und schriftlicher Aufklärung sorgt für mehr Transparenz gegenüber den Eltern und verbessert somit auch die prä- und postoperative Befindlichkeit der Patienten und ihrer Angehörigen. Dementsprechend ist es notwendig in Zukunft mündliche Handlungsanleitungen auch in schriftlicher Form zur Verfügung zu stellen. Hierzu sollte ein standardisierter Behandlungsbogen entwickelt werden, der bestenfalls auch außerhalb der Kinderchirurgie eingesetzt wird.

Ziel sollte es außerdem sein, die Eltern hinsichtlich postoperativ auftretender Schmerzen zu sensibilisieren und eine ausreichende analgetische Therapie sicherzustellen. Eine Steigerung der verlässlichen Schmerzmittelgabe durch die Eltern konnte durch die schriftliche Anweisung bereits erwirkt werden. Trotzdem sollte eine weitere Optimierung Bestandteil zukünftiger Forschungsprojekte sein.

Gerade in der Kinderchirurgie ist ein reibungsloser Ablauf des Operationstages sehr wichtig, um traumatische Erlebnisse gänzlich zu vermeiden. Die Perfektionierung der Abläufe sollte Schwerpunkt und Ziel des behandelnden Teams sein. 


\section{$6 \quad$ Anhang}

\subsection{Fragebogen präoperativ}

Fallnummer:

„Auswirkungen eines geführten perioperativen Behandlungskonzepts bei ambulant zu operierenden Kindern auf die prä- und postoperative Befindlichkeit“"

Bitte beantworten Sie die nachfolgenden Fragen ...

Welche Operation bekommt ihr Kind?

Bitte schreiben Sie ihre Antwort in den folgenden Kasten.

Warum haben Sie sich für die ambulante Operation entschieden?

Bitte schreiben Sie ihre Antwort in den folgenden Kasten. 
Bitte kreuzen Sie das zutreffende Kästchen an.

\begin{tabular}{l|l|l|l|l|l|} 
& & & & & \\
\hline $\begin{array}{l}\text { Ich bin über Alternativen zur ambulanten Operation } \\
\text { aufgeklärt worden. }\end{array}$ & & & & & \\
\hline $\begin{array}{l}\text { Ich weiß, wo und wann ich mich an dem Tag der } \\
\text { Operation im Krankenhaus einfinden soll. }\end{array}$ & & & & & \\
\hline $\begin{array}{l}\text { Der Tagesablauf am OP Tag ist mir ausführlich } \\
\text { erklärt worden. }\end{array}$ & & & & & \\
\hline $\begin{array}{l}\text { Mir ist bekannt, wann mein Kind das letzte Mal etwas } \\
\text { Essen und Trinken darf. }\end{array}$ & & & & & \\
\hline $\begin{array}{l}\text { Bitte geben sie hier die Uhrzeit } \\
\text { an: }\end{array}$ & & & & & \\
\hline $\begin{array}{l}\text { Ich bin über die Schmerztherapie meines Kindes } \\
\text { nach Hause kann ich problemlos organisieren. }\end{array}$ & & & & & \\
\hline
\end{tabular}

\section{Was mich außerdem noch beschäftigt ...}

Bitte schreiben Sie ihre Antwort in den folgenden Kasten 


\subsection{Präoperativer Informationsbogen}

Auswirkungen eines geführten perioperativen Behandlungskonzepts bei ambulant zu operierenden Kindern auf die prä- und postoperative Befindlichkeit.

Liebe Eltern,

ihr Kind wird in naher Zukunft im operativen Kinderzentrum der Universitätsmedizin Göttingen ambulant operiert. Dies ist sowohl für ihr Kind als auch für Sie meist eine neue, vielleicht auch anstrengende Situation. Vor diesem Hintergrund möchten wir die Qualität der Vorbereitung auf diesen Tag mit Hilfe einer Umfrage prüfen, um so die Aufklärung der Eltern bezüglich der Versorgung der Kinder zu Hause und den reibungslosen Ablauf am Tag der Operation zu verbessern. Hierzu möchten Wir Sie bitten, diesen Fragebogen auszufüllen und zum Tag der Operation wieder mit ins Krankenhaus zubringen. Da es erst im Anschluss an diese Umfrage zu eventuellen Veränderungen des Behandlungskonzeptes kommen wird, entsteht für ihr Kind kein direkter Nutzen aus der Studienteilnahme. Selbstverständlich ist die Teilnahme an der Studie freiwillig, eine Nichtteilnahme bringt keine Nachteile für Sie mit sich.

Zusätzlich zu diesem Bogen werden der Patientenakte Ihres Kindes ausgewählte personenbezogene Informationen bezüglich der Narkose, Medikation und Operation entnommen und in pseudonymisierter Form aufgezeichnet. Pseudonymisierung bedeutet Verschlüsselung von Daten ohne Namensnennung nur mit Nummern codiert. Die Zuordnung der Daten oder Proben zu einer Person ist nur möglich, wenn hierfür der Schlüssel eingesetzt wird, mit dem die Daten pseudonymisiert (verschlüsselt) wurden. Die personenbezogenen Daten werden unter besonderen Schutzvorkehrung getrennt von der Schlüsselliste aufbewahrt. Eine Entschlüsselung ist nur durch die verantwortlichen Studienärzte möglich. Dritte erhalten keinen Einblick in die Unterlagen. Die gesammelten Daten aus den Akten und den Fragebögen werden statistisch ausgewertet.

Vielen Dank für Ihre freundliche Unterstützung! 


\subsection{Fragebogen postoperativ, Tag 1}

Die postoperativen Fragebögen wurden telefonisch durchgeführt und die Antwortmöglichkeiten in eine Datentabelle übertragen. Im Folgenden eine Aufzählung der gestellten Fragen.

Frage: Wurde ihr Kind schon einmal operiert?

Voroperationen:

Folgende körperliche Symptome sind am Operationstag, oder am Tag danach aufgetreten:

\begin{tabular}{|l|l|l|}
\hline Symptom & Ja & Nein \\
\hline Schmerzen & & \\
\hline Übelkeit & & \\
\hline Erbrechen & & \\
\hline Blutungen & & \\
\hline Müdigkeit & & \\
\hline
\end{tabular}

\begin{tabular}{|c|c|c|c|c|c|}
\hline Items & 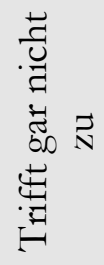 & 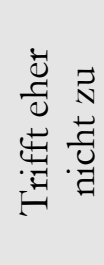 & 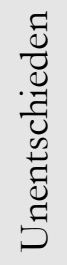 & 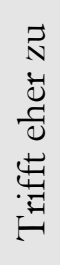 & 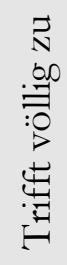 \\
\hline $\begin{array}{l}\text { Mein Kind hat nach der Operation im Krankenhaus } \\
\text { noch zusätzliche Schmerzmittel benötigt. }\end{array}$ & & & & & \\
\hline $\begin{array}{l}\text { Ich bin mit der Versorgung meines Kindes im } \\
\text { Krankenhaus zufrieden. }\end{array}$ & & & & & \\
\hline $\begin{array}{l}\text { Ich bin ausreichend über eine mögliche } \\
\text { Schmerzmedikation zu Hause informiert worden. }\end{array}$ & & & & & \\
\hline $\begin{array}{l}\text { Ich konnte mit der Schmerzsituation meines Kindes } \\
\text { erfolgreich umgehen. }\end{array}$ & & & & & \\
\hline $\begin{array}{l}\text { Bei meinem Kind sind postoperativ Nachblutungen } \\
\text { aufgetreten. }\end{array}$ & & & & & \\
\hline $\begin{array}{l}\text { Ich bin vor der Operation ausreichend über eventuell } \\
\text { auftretende Nachblutungen informiert worden. }\end{array}$ & & & & & \\
\hline $\begin{array}{l}\text { Ich wusste wie ich mich dem Falle einer Nachblutung } \\
\text { verhalten soll. }\end{array}$ & & & & & \\
\hline
\end{tabular}


Ich wurde über verschiedene Narkoseverfahren aufgeklärt.

Ich wurde ausreichend über das gewählte Narkoseverfahren aufgeklärt.

Mein Kind hat die Narkose gut vertragen.

Meinem Kund wurde für die Zeit der Operation ein Blasenkatheter gelegt.

Hat Ihr Kind das Trinken gut vertragen?

Hat Ihr Kind das Essen gut vertragen?

Der Pflasterwechsel bei meinem Kind verlief problemlos.

Die Wundheilung verlief bei meinem Kind problemlos.

Ich wurde ausreichend über die Vor- und Nachteile einer ambulanten Operation ausgeklärt.

Mir wurde die Option mein Kind stationär operieren zu lassen angeboten.

Meine Wünsche wurden bei der Terminvergabe berücksichtigt.

Würden Sie ihr Kind nocheinmal ambulant operieren lassen?

Wenn nicht, warum nicht?

Hat die Besprechung mit den Ärzten nach der Operation alle Ihre Fragen beantwortet?

Wenn nicht, welche Informationen haben Ihnne gefehlt?

Waren Sie mit dem Ablauf im Krankenhaus am Tag der Operation zufrieden?

Wenn nicht, warum nicht?

Wussten Sie, wo Sie sich morgens vor der Operation melden müssen?

Mussten Sie und Ihr Kind lange bis zur Narkoseeinleitung warten?

Wurde die angegebene Operationszeit eingehalten?

Der Transport meines Kindes nach der Operation verlief komplikationslos.

Wenn nicht, warum nicht?

Ich halte die Nachbetreuung durch den Kinderarzt für eine gute Idee.

Ich hätte mir einen Nachbesprechungstermin in der Klinik gewünscht. 


\section{Fragen}

Welche Medikamente haben Sie verabreicht?

Wann haben sie die Medikamente verabreicht?

Wie oft haben Sie die Medikamente verabreicht?

Wie lange hat die Operation gedauert?

Wie viele Stunden nach der Operation hat ihr Kind das erste Mal wieder etwas getrunken?

Wie viele Stunden nach der Operation hat ihr Kind das erste Mal wieder Nahrung zu sich genommen?

Wie viele Stunden nach der Operation hat ihr Kind das erste Mal Urin gelassen?

Nach welchem Zeitraum war ihr Kind komplett beschwerdefrei? (Er/Sie hat wieder normal gespielt und getobt?)

Wie lange mussten Sie nach der Operation im Krankenhaus warten?

Welches Transportmittel haben sie gewählt?

\subsection{Fragebogen postoperativ, Tag 3}

Auch hier erfolgte eine telefonische Befragung. Im Folgenden wiederum die Items.

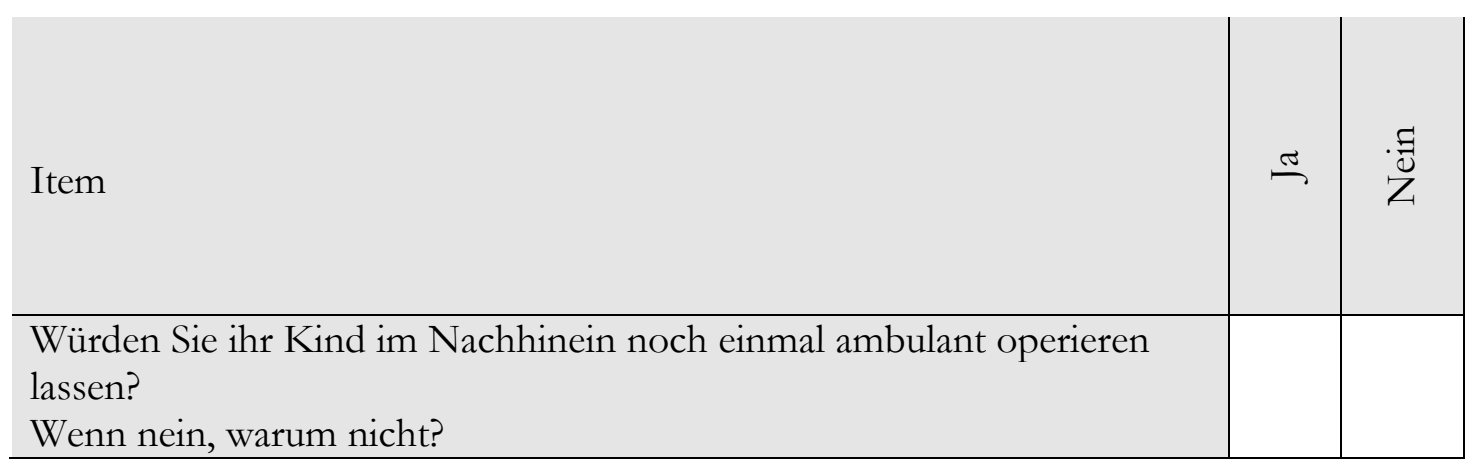




\begin{tabular}{|c|c|c|c|c|c|}
\hline Items & 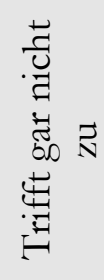 & 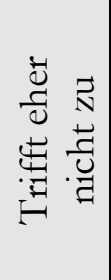 & 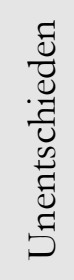 & 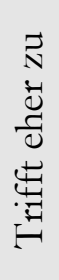 & 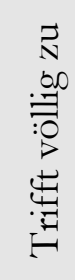 \\
\hline $\begin{array}{l}\text { Der Tag der Operation war meiner Ansicht nach klar } \\
\text { strukturiert und für mich vorhersehbar. }\end{array}$ & & & & & \\
\hline $\begin{array}{l}\text { Am Tag der Operation hatte ich das Gefühl, dass } \\
\text { mein Kind sich in guten Händen befindet. }\end{array}$ & & & & & \\
\hline $\begin{array}{l}\text { In Vorbereitung auf die Operation hätte ich mir eine } \\
\text { detailliertere Aufklärung gewünscht. }\end{array}$ & & & & & \\
\hline $\begin{array}{l}\text { Am Tag der Operation war ich der Verantwortung } \\
\text { für mein Kind gewachsen. }\end{array}$ & & & & & \\
\hline $\begin{array}{l}\text { Ich wusste an welche Person ich mich im Falle von } \\
\text { Fragen oder Problemen wenden konnte. }\end{array}$ & & & & & \\
\hline $\begin{array}{l}\text { Ich halte die Nachsorgeuntersuchung beim } \\
\text { Kinderarzt für eine gute Idee. }\end{array}$ & & & & & \\
\hline $\begin{array}{l}\text { Ich bin mit der Betreuung durch die Pflegekräfte in } \\
\text { der Aufnahme zufrieden. }\end{array}$ & & & & & \\
\hline $\begin{array}{l}\text { Ich bin mit der Betreuung durch die in dem } \\
\text { Wartezimmer zufrieden. }\end{array}$ & & & & & \\
\hline $\begin{array}{l}\text { Ich bin mit der Betreuung durch die Pflegekräfte im } \\
\text { Aufwachraum zufrieden. }\end{array}$ & & & & & \\
\hline $\begin{array}{l}\text { Ich bin mit der Betreuung durch das Ärzteteam } \\
\text { zufrieden. }\end{array}$ & & & & & \\
\hline
\end{tabular}




\section{$7 \quad$ Literaturverzeichnis}

Alp BF, Irkilata HC, Kibar Y, Zorba U, Sancaktutar AA, Kaya E, Dayanc M (2014): Comparison of the inguinal and scrotal approaches for the treatment of communicating hydrocele in children. Kaohsiung J Med Sci 30, 200-205

Andersson V, Otterstrom-Rydberg E, Karlsson AK (2015): The Importance of Written and Verbal Information on Pain Treatment for Patients Undergoing Surgical Interventions. Pain Manag Nurs $\underline{16}$, 634-641

Beyaz SG, Tokgöz O, Tüfek A (2011): Caudal epidural block in children and infants: retrospective analysis of 2088 cases. Ann Saudi Med 31, 494-497

Brenn BR, Choudhry DK, Sacks K (2016): Outpatient outcomes and satisfaction in pediatric population: data from the postoperative phone call. Paediatr Anaesth 26, 158-163

Brosius KK, Bannister CF (2002): Oral midazolam premedication in preadolescents and adolescents. Anesth Analg 94, 31-36

Chan L, Russell TJ, Robak N (1998): Parental perception of the adequacy of pain control in their child after discharge from the emergency department. Pediatr Emerg Care 14, 251-253

Comley AK, DeMeyer E (2001): Assessing patient satisfaction with pain management through a continuous quality improvement effort. J Pain Symptom Manag 21, 27-40

Coyne I (2006): Children’s experiences of hospitalization. J Child Health Care 10, 326-336

Deer JD, Sawardekar A, Suresh S (2016): Day surgery regional anesthesia in children: safety and improving outcomes, do they make a difference? Curr Opin Anaesthesiol 29, 691-695

Deindl C (Hrsg.): Manual Ambulantes Operieren: Techniken, perioperative Verfahren und Management. Walter de Gruyter GmbH \& Co KG, Berlin/Boston 2016

Deshmukh PV, Kulkarni SS, Parchandekar MK, Sikchi SP (2016): Comparison of preanesthetic sedation in pediatric patients with oral and intranasal midazolam. J Anaesthesiol Clin Pharmacol 32, 353-358

Dewan PA, Tieu HC, Chieng BS (1996): Phimosis: is circumcision necessary? J Paediatr Child Health $\underline{32}, 285-289$

Dick WF (1997): Die Anästhesieambulanz - Lösung des Problems? Anaesthesist 46, S96-S98

Ellerton ML, Merriam C (1994): Preparing children and families psychologically for day surgery: an evaluation. J Adv Nurs $\underline{19}$, 1057-1062

Esposito C, Montinaro L, Alicchio F, Scermino S, Basile A, Armenise T, Settimi A (2009):

Technical standardization of laparoscopic herniorraphy in pediatric patients. World J Surg 33, 1846-1850

Esposito C, Escolino M, Cortese G, Aprea G, Turrà F, Farina A, Roberti A, Cerulo M, Settimi A (2017): Twenty-year experience with laparoscopic inguinal hernia repair in infants and children: considerations and results on 1833 hernia repairs. Surg Endosc 31, 1461-1468

Fabricant PD, Seeley MA, Rozell JC, Flynn JM, Wells LM, Ganley TJ 2016): Cost Savings From Utilization of an Ambulatory Sugery Center for Orthopaedic Day Sugery. J Am Acad Orthop Surg 24, 865-871 
Fechner G (2011): Operations with scrotal approach. Aktuel Urol 42, 197-213

Fincher W, Shaw J, Ramelet AS (2012): The effectiveness of a standardised preoperative preparation in reducing child and parent anxiety: a single-blind randomised controlled trial. J Clin Nurs 21, 946-955

Finley GA, Chambers CT, McGrath PJ, Walsh TM (2003): Construct validity of the parents' postoperative pain measure. Clin J Pain 329-334

Forward SP, Brown TL, McGrath PJ (1996): Mothers' attitudes and behavior toward medicating children's pain. Pain $\underline{67}, 469-474$

Gill FT (1998): Umbilical hernia, inguinal hernias, and hydroceles in children: diagnostic clues for optimal patient management. J Pediatr Health Care 12, 231-235

Göbel P, Stuhldreier G (2008): Inguinal hernia, and hydrocele-special features in infants and children. Zentralbl Chir 133, 531-534

Hamers JPH, Abu-Saad HH (2002): Children's pain at home following (adeno) tonsillectomy. Eur J Pain $\underline{6}, 213-219$

Hautmann R, Huland H (Hrsg.): Urologie. 3. Auflage; Springer Medizin Verlag, Heidelberg 2006

Hegarty M, Calder A, Davies K, Shave M, Christiansen E, Meyer T, von Ungern-Sternberg BS (2013): Does take-home analgesia improve postoperative pain after elective day case surgery? A comparison of hospital vs parent-supplied analgesia. Paediatr Anaesth $\underline{23}$, 385389

Hrivatakis G, Astfalk W, Schmidt A, Hartwig A, Kugler T, Heim T, Clausner A, Frunder A, Weber $\mathrm{H}$, Loff $\mathrm{S}$ et al. (2014): The timing of surgery for undescended testis - a retrospective multicenter analysis. Dtsch Arztebl Int 111, 649-657

Hutcheson JC, Cooper CS, Snyder HM (2000): The anatomical approach to inguinal orchiopexy. J Urol $\underline{164}, 1702-1704$

Jonas DA (2003): Parent's management of their child's pain in the home following day surgery. J Child Health Care 7, 150-162

Kain ZN, Mayes LC, Wang SM, Caramico LA, Krivutza DM, Hofstadter MB (2000): Parental presence and a sedative premedicant for children undergoing surgery: a hierarchical study. Anesthesiology 92, 939-946

Kain ZN, Mayes LC, Caramico LA (1996): Preoperative preparation in children: a cross-sectional study. J Clin Anesth $\underline{8}, 508-514$

Kankkunen P, Vehviläinen-Julkunen K, Pietilä AM, Halonen P (2003): Is the sufficiency of discharge instructions related to children's postoperative pain at home after day surgery? Scand J Caring Sci 17, 365-372

Kankkunen PM, Vehviläinen-Julkunen KM, Pietilä AM (2002): Children's postoperative pain at home: family interview study. Int J Nurs Pract $\underline{8}, 32-41$

Karl A, Blasl H, Stief C, Riccabona M (2016): Ambulantes Operieren im Kindesalter. Urologe $\underline{55}$, $19-26$ 
Kaya Z, Süren M, Arici S, Karaman S, Tapar H, Erdemir F (2012): Prospective, randomized, double-blinded comparison of the effects of caudally administered levobupivacaine $0.25 \%$ and bupivacaine $0.25 \%$ on pain and motor block in children undergoing circumcision surgery. Eur Rev Med Pharmacol Sci 16, 2014-2020

Kliesch S (2014): Hydrocele, spermatocele, and vasectomy: management of complications. Urologe $\underline{53}, 671-675$

Kollin C, Karpe B, Hesser U, Granholm T, Ritzén EM (2007): Surgical treatment of unilaterally undescended testes: testicular growth after randomization to orchiopexy at age 9 months or 3 years. J Urol $\underline{178}, 1589-1593$

Kostner S (2012): Hydrozele im Jugendalter. https://www.online-zfa.de/article/hydrozele-imjugendalter/ebm/y/m/1146; abgerufen am 04.04.2017

Kovac AL (2007): Management of postoperative nausea and vomiting in children. Paediatr Drugs 9., 47-69

Landier M, Villemagne T, Le Touze A, Braik K, Meignan P, Cook AR, Morel B, Lardy H, Binet A (2017): The position of a written document in preoperative information for pediatric surgery: A randomized controlled trial on parental anxiety, knowledge and satisfaction. J Pediatr Surg $\underline{53}$, 375380

Lao OB, Fitzgibbons RJ, Cusick RA (2012): Pediatric inguinal hernias, hydroceles, and undescended testicles. Surg Clin North Am 92, 487-504

Li HCW, Lopez V, Lee TLI (2007): Psychoeducational preparation of children for surgery: the importance of parental involvement. Patient Educ Couns $\underline{65}, 34-41$

Liu W, Wu R, Du G (2014): Single-port laparoscopic extraperitoneal repair of pediatric inguinal hernias and hydroceles by using modified Kirschner pin: a novel technique. Hernia 18, 345-349

Longard J, Twycross A, Williams AM, Hong P, Chorney J (2016): Parents' experiences of managing their child's postoperative pain at home: an exploratory qualitative study. J Clinic Nurs $\underline{25}$, 2619-2628

Lynch M (1994): Preparing children for day surgery. Child Health Care $\underline{23}$, 75-85

Machotta A (2014): Parental presence during induction of anesthesia in children: pros and cons. Anaesthesist 63, 326-330

MacLaren Chorney J, Twycross A, Mifflin K, Archibald K (2014): Can we improve parents' management of their children's postoperative pain at home? Pain Res Manag $\underline{19}, 115-123$

Maddox MM, Smith DP (2008): A long-term prospective analysis of pediatric unilateral inguinal hernias: should laparoscopy or anything else influence the management of the contralateral side? J Pediatr Urol 4, 141-145

Mani V, Morton NS (2010): Overview of total intravenous anesthesia in children. Paediatr Anaesth $\underline{20}, 211-222$

Marechal C, Berthiller J, Tosetti S, Cogniat B, Desombres H, Bouvet L, Kassai B, Chassard D, de Querioz Siqueira M (2017): Children and parental anxiolysis in paediatric ambulatory surgery: a randomized controlled study comparing $0.3 \mathrm{mg} \mathrm{kg}-1$ midazolam to tablet computer based interactive distraction. Br J Anaesth $\underline{118}, 247-253$ 
Messeri A, Caprilli S, Busoni P (2004): Anaesthesia induction in children: a psychological evaluation of the efficiency of parents' presence. Paediatr Anaesth 14, 551-556

Miguel Romeo MC, Sagardoy Muniesa L (2014): Effects of using an information leaflet in reducing perioperative anxiety and pain in patients undergoing urological surgery. Enferm Clin $\underline{24}$, 233-240

Misra D, Dias R, Kapila L (1997): Scrotal fixation: a different surgical approach in the management of the low undescended testes. Urology 49, 762-765

Mohey A, Gharib TM, Omar RG, Sebaey A, Elmohamady BN, Kandeel W (2017): High single scrotal-incision orchidopexy as the standard technique in infants aged 6-24 months. Arab J Urol 15, 78-81

Müller Markus (Hrsg.): Chirurgie für Studium und Praxis. 13. Auflage; Medizinische Verlags- und Informationsdienste, Breisach 2016/2017

Naji H, Ingolfsson I, Isacson D, Svensson JF (2012): Decision making in the management of hydroceles in infants and children. Eur J Pediatr 171, 807-810

Niyogi A, Tahim AS, Sherwood WJ, De Caluwe D, Madden NP, Abel RM, Haddad MJ, Clarke SA (2010): A comparative study examining open inguinal herniotomy with and without hernioscopy to laparoscopic inguinal hernia repair in a pediatric population. Pediatr Surg Int $\underline{26}, 387-392$

Aumüller G, Aust G, Engele J, Kirsch J, Maio G, Mayerhofer A, Mense S, Reißig D, Salvetter J, Schmidt W et al. (2014): Duale Reihe Anatomie. 3. Auflage; Georg Thieme Verlag. Stuttgart 2014

BAO: Ambulantes operieren. Voraussetzungen. Bundesverband für ambulantes Operieren e.V. https://www.operieren.de/e3224/e308/e331/e334/index_html; abgerufen am 08.07.2018]

Oddby E, Englund S, Lönnqvist PA (2001): Postoperative nausea and vomiting in paediatric ambulatory surgery: sevoflurane versus spinal anaesthesia with propofol sedation. Paediatr Anaesth 11, 337-342

Ortiz AC, Attalah AN, Matos D, da Silva EM (2014): Intravenous versus inhalational anaesthesia for pediatric outpatient surgery. Cochrane Database of Syst Rev 2, CD009015

Pandit SK, Green CR (1994): General anesthetic techniques. Int Anesthesiol Clin 32, 55-79

Paquette J, Le May S, Lachance Fiola J, Villeneuve E, Lapointe A, Bourgault P (2013): A randomized clinical trial of a nurse telephone follow-up on paediatric tonsillectomy pain management and complications. J Adv Nurs $\underline{69}$, 2054-2065

Potts WJ, Riker WL, Lewis JE (1950): The treatment of inguinal hernia in infants and children. Ann Surg $\underline{132}, 566-576$

Reich A, Brinkmann OA (2004): Regional anesthesia for urologic interventions in the pediatric age group. Aktuelle Urol. $\underline{35}, 418-425$

Rony RYZ, Fortier MA, Chorney JM, Perret D, Kain ZN (2010): Parental postoperative pain management: attitudes, assessment, and management. Pediatrics $\underline{125}, 1372-1378$

Russell P, von Ungern-Sternberg BR, Schug SA(2013): Perioperative analgesia in pediatric surgery. Curr Opin Anaesthesiol 26, 420-427 
Sartori J, Espinoza P, Díaz MS, Ferdinand C, Lacassi HJ, González A et al. (2015): What preoperative information do the parents of children undergoing surgery want? Rev Chil Pediatr 86, 399-403

Sjöling M, Nordahl G, Olofsson N, Asplund K (2003): The impact of preoperative information on state anxiety, postoperative pain and satisfaction with pain management. Patient Educ Couns $\underline{51}, 169-176$

Sökeland J, Rübben H (Hrsg.): Urologie. 14. Auflage; Georg Thieme Verlag, Stuttgart 2007

Spencer C, Franck LS (2005): Giving parents written information about children's anesthesia: are setting and timing important? Paediatr Anaesth $\underline{15}, 547-553$

Stark E, Steffens J (2006): The state of pediatric urological outpatient surgery. Aktuelle Urol $\underline{37}$, 127-131

Stein ALS, Baumgard D, Del Rio I, Tutiven JL (2017): Updates in Pediatric Regional Anesthesia and Its Role in the Treatment of Acute Pain in the Ambulatory Setting. Curr Pain Headache Rep 21, 11

Steward DJ (2014): The origins and development of pediatric outpatient surgery. Paediatr Anaesth $\underline{24}, 632-634$

Unsworth V, Franck LS, Choonara I (2007): Parental assessment and management of children's postoperative pain: a randomized clinical trial. J Child Health Care 11, 186-194

Urman RD, Desai SP (2012): History of anesthesia for ambulatory surgery. Curr Opin Anaesthesiol $\underline{25}, 641-647$

Wang KS, Committee on Fetus and Newborn, American Academy of Pediatrics und Section on Surgery, American Academy of Pediatrics (2012): Assessment and management of inguinal hernia in infants. Pediatrics $\underline{130}, 768-773$

Ygge BM, Arnetz JE (2001): Quality of paediatric care: application and validation of an instrument for measuring parent satisfaction with hospital care. Int J Qual Health Care 13, 33-43 